\title{
Terahertz Aqueous Photonics
}

\author{
by \\ Qi Jin \\ Submitted in Partial Fulfillment of the \\ Requirements for the Degree \\ Doctor of Philosophy
}

Supervised by

Professor Xi-Cheng Zhang

The Institute of Optics

Arts, Sciences and Engineering

Edmund A. Hajim School of Engineering and Applied Sciences

\author{
University of Rochester \\ Rochester, New York
}


Dedicated to my parents 


\section{Table of Contents}

Biographical Sketch vi v vi v v

\begin{tabular}{|c|c|}
\hline Acknowledgments & $\mathrm{x}$ \\
\hline
\end{tabular}

Abstract xii

Contributors and Funding Sources xiv

List of Figures $\quad$ xvi

\begin{tabular}{lll}
\hline & Introduction & 1
\end{tabular}

1.1 Terahertz Frequency Band . . . . . . . . . . . . . . . . . 1

1.2 Properties and Applications . . . . . . . . . . . . . . . . . . . 3

1.3 Pulsed $\mathrm{THz}$ sources . . . . . . . . . . . . . . . . . . . . . . . . 7

1.3.1 Photoconductive Antenna . . . . . . . . . . . . . 7

1.3 .2 Nonlinear Crystal . . . . . . . . . . . . . . . . . . 8

$1.3 .3 \mathrm{Gas} \ldots \ldots \ldots \ldots \ldots$

1.3 .4 Magnetic Heterostructure . . . . . . . . . . . 11 
1.4 Success of Using Liquid Water as the Source in Other Frequency Regions . . . . . . . . . . . . . . . . . . . 12

1.4.1 Supercontinuum Generation from Water . . . . . . . . 13

1.4 .2 XUV Generation from Water . . . . . . . . . . . . . 16

1.5 Challenges and Opportunities . . . . . . . . . . . . 20

2 Terahertz Wave Generation from a Water Film 23

$2.1 \quad$ Free-Flowing Thin Water Film . . . . . . . . . . . . . . . . . 23

2.2 Experimental Set-up . . . . . . . . . . . . . . . . 26

2.3 Terahertz Radiation from a Water Film . . . . . . . . . . . . . 27

2.4 Comparison between Terahertz Radiation from Water and Air 31

2.5 Effect of Optical Polarization and Pulse Energy . . . . . . . . 32

2.6 Effect of Water Film's Thickness . . . . . . . . . . . . . . . . 34

2.7 Forced-Flowing Thin Water Film . . . . . . . . . . . . . . . . 37

2.8 Mechanism of the Generation Process . . . . . . . . . . . . . . 38

2.8 .1 Laser-Induced Plasma Formation . . . . . . . . . . . . 40

2.8 .2 Dipole Radiation Model . . . . . . . . . . . . . . . . 44

2.9 Radiation Pattern . . . . . . . . . . . . . . . . . . . . . 46

2.10 Effect of Optical Pulse Duration . . . . . . . . . . . . . . 51

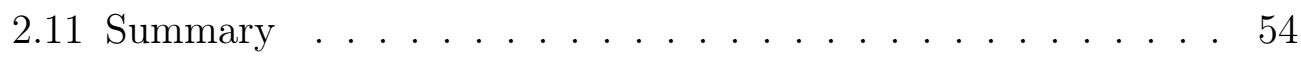

3 Terahertz Radiation from Liquid Water under Two-Color Ex$\begin{array}{lr}\text { citation Scheme } & 56\end{array}$

3.1 Glory of Two-Color Excitation Scheme in Gases . . . . . . . . 57 
3.2 Experimental Setup of the Two-Color Excitation Scheme . . . 63

3.3 Comparison between Terahertz Radiation from One-Color and Two-Color Excitation Scheme . . . . . . . . . . . . . . . . 65

3.4 Modulation of $\mathrm{THz}$ Fields . . . . . . . . . . . . . . . . . 67

3.5 Discussion about Contributions from Water and Air . . . . . . 72

3.6 Summary $\ldots \ldots \ldots \ldots$. . . . . . . . . . . . . . . 74

4 Terahertz Wave Generation from a Water Line 75

$4.1 \quad$ Issues of Using Water Films . . . . . . . . . . . . . . . . . . 75

4.2 Water Lines . . . . . . . . . . . . . . . . . . . 76

$4.3 \mathrm{THz}$ Radiation from the Water Line . . . . . . . . . . . . . . . 79

4.4 Preference for Sub-Picosecond Laser Pulses . . . . . . . . . . . 82

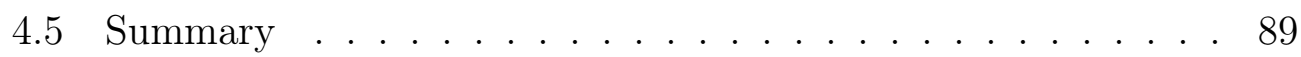

5 Terahertz Wave Generation from $\alpha$-Pinene 91

$5.1 \quad \alpha$-Pinene . . . . . . . . . . . . . . . . . . . . . 91

5.2 Comparison between Terahertz Radiation from $\alpha$-Pinene and Water ........................ 94

5.3 Summary . . . . . . . . . . . . . . . . . 96

\begin{tabular}{lll}
\hline & Conclusion and Future Work & 98
\end{tabular}

\begin{tabular}{lr}
\hline Bibliography & 101
\end{tabular} 


\section{Biographical Sketch}

The author was born in Wuhan, Hubei, the People's Republic of China in 1990. He attended Huazhong University of Science and Technology and graduated with a Bachelor of Science degree in Optoelectronic Science and Engineering in 2012. In May 2017, he acquired a Master of Science degree in Optics at the University of Rochester. He began his doctoral studies in Optics at the University of Rochester in June 2017. He joined Professor Xi-Cheng Zhang's group to pursue research in terahertz science and technology.

In 2016, he received the James Fienup Academic Achievement Award from The Institute of Optics. In 2017, he was awarded Outstanding Student Paper at the IRMMW-THz conference in Mexico. In 2018, he won the Rochester Precision Optics Graduate Project Award for his work in broadband terahertz wave generation from liquid water. 


\section{Publications}

\section{Journals}

1. Q. Jin, Y. E, S. Gao, and X. -C. Zhang. "Investigation on the preference of sub-picosecond laser pulses for terahertz wave generation from liquids". To be submitted.

2. Y. E, Q. Jin, and X. -C. Zhang. "Enhancement of terahertz emission by a preformed plasma in liquid water". Applied Physics Letters, 115 (10), 101101 (2019).

3. Q. Jin, J. Dai, Y. E, and X. -C. Zhang. "Terahertz wave emission from a liquid water film under the excitation of asymmetric optical fields". Applied Physics Letters, 113 (26), 261101 (2018). (Featured article)

4. Y. E, Q. Jin, A. Tcypkin, and X. -C. Zhang. "Generation of terahertz wave from liquid water films via laser-induced breakdown". Applied Physics Letters, 113 (18), 181103 (2018).

5. Q. Jin, Y. E, K. Williams, J. Dai, and X. -C. Zhang. "Observation of broadband terahertz wave generation from liquid water". Applied Physics Letters, 111 (7), 071103 (2017).

6. M. Esaulkov, P. Solyankin, A. Sidorov, L. Parshina, A. Makarevich, Q. Jin, Q. Luo, O. Novodvorsky, A. Kaul, E. Cherepetskaya, A. Shkurinov, V. Makarov, X. -C. Zhang. "Emission of Terahertz pulses from 
vanadium dioxide films undergoing metal-insulator phase transition". Optica, 2 (9), 790-796 (2015).

\section{Conference Proceedings}

1. Q. Jin, Y. E, J. Dai, L. Zhang, C. Zhang, A. Tcypkin, S. Kozlov, and X. -C. Zhang. "Terahertz photonics in liquids". Nonlinear Optics, NTu2B.3 (OSA 2019).

2. Y. E, Q. Jin, and X. -C. Zhang. "Enhanced emission of terahertz wave from liquid water". SPIE/COS Photonics Asia, 11196-6 (SPIE 2019).

3. S. Gao, Q. Jin, Y. E, and X. -C. Zhang. "Investigation of different liquid properties on emitting $\mathrm{THz}$ wave under ultrashort optical excitation". SPIE/COS Photonics Asia, 11196-8 (SPIE 2019).

4. Q. Jin, Y. E, L. Zhang, C. Zhang, A. Tcypkin, S. Kozlov, and X. C. Zhang. "THz aqueous photonics and beyond". 43nd International Conference on Infrared, Millimeter, and Terahertz Waves (IEEE 2018).

5. J. Dai, Q. Jin, Y. E, K. Williams, and X. -C. Zhang. "Using liquid water as broadband terahertz wave emitter". Image Sensing Technologies: Materials, Devices, Systems, and Applications V, 106561L (SPIE 2018).

6. Y. E, Q. Jin, K. Williams, J. Dai, and X. -C. Zhang. "Terahertz wave generation from water". CLEO: Science and Innovations, SM4A. 1 
(OSA 2018).

7. X. -C. Zhang, Y. E, Q. Jin, A. Tcypkin, and L. Zhang. "Recent development on $\mathrm{THz}$ aqueous photonics". 9th International Symposium on Ultrafast Phenomena and Terahertz Waves, ThA1 (OSA 2018).

8. Q. Jin, K. Williams, Y. E, J. Dai, and X. -C. Zhang. "Observation of broadband terahertz wave generation from liquid water". 42nd International Conference on Infrared, Millimeter, and Terahertz Waves (IEEE 2017).

9. Q. Jin, K. Williams, Y. E, J. Dai, and X. -C. Zhang. "Observation of broadband terahertz wave generation from liquid water". Nonlinear Optics, NW3A.1 (OSA 2017).

10. Y. E, Q. Jin, K. Williams, J. Dai, and X. -C. Zhang. "THz wave emission from water". RJUSE TeraTech (2017).

11. Q. Jin, K. Williams, Y. E, J. Dai, and X. -C. Zhang. "Terahertz wave generation from liquid water". THz Bio Conference (2017). 


\section{Acknowledgments}

Pursuing a PhD degree in the United Stated was not an easy decision for me to make. The admiration for my advisor Professor Xi-Cheng Zhang was the main reason that drove me to spend years learning optics and terahertz technology at The Institute of Optics. Besides his success in the terahertz community, the care and the respect that he had shown to students deeply touched me. I am very grateful for the excellent academic environment and priceless advice he has offered to support my research. He inspired me to work smart, which helped me to make breakthroughs during my $\mathrm{PhD}$ study. I believe that the lessons I have learned from him will significantly impact on the rest of my life.

I would like to thank other members of my thesis committee Professor Wayne Knox and Professor Roman Sobolewski, and the committee chair Professor Yongli Gao for reviewing my work.

I appreciate the help from present and past group members in Professor Zhang's group. Yiwen E, our postdoc, helped to solve tremendous problems in my research. Kang Liu, Fabrizio Buccheri, and Betty Sun offered me many 
suggestions when I started the project for my thesis. Kaia Williams helped preparing early experiments. Kareem Garriga, Jiapeng Zhao, and Shenghan Gao provided insightful ideas in our discussions.

Special thanks to Professor Jianming Dai for showing me how to professionally carry out an experiment, as well as offering me advice on my career and life.

I am fortunate to have met the staff in The Institute of Optics: Kai Davies, Lori Russell, Adrienne Snopkowski, and Kari Brick. With their dedicated work, I could fully focus on my research.

Last words are for my dear parents. They are always on my side supporting me. Without their sacrifices, I will never be the person I want to be. 


\section{Abstract}

Developing efficient and robust terahertz $(\mathrm{THz})$ sources is of incessant interest in the $\mathrm{THz}$ community for their wide applications. For instance, $\mathrm{THz}$ waves are widely applied to nondestructive evaluation and biomedical imaging. Intense $\mathrm{THz}$ fields can be used for nonlinear interactions and electron acceleration. With successive efforts in past decades, numerous groups have achieved $\mathrm{THz}$ wave generation from solids, gases, and plasmas. However, liquid, especially liquid water has never been demonstrated as a THz source. One main reason leading the impediment is that water has strong absorption characteristics in the $\mathrm{THz}$ frequency regime. In this dissertation, a thin water film under intense laser excitation was introduced as the $\mathrm{THz}$ source to mitigate the considerable loss of $\mathrm{THz}$ waves from the absorption. Laserinduced plasma formation associated with a ponderomotive force-induced dipole model was proposed to explain the generation process. Compared with gas targets, water has a lower photon-ionization energy, but three orders of magnitude higher molecular density. Compared with solid targets, water does not suffer from permanent damage caused by the intense laser 
pulses.

For the one-color excitation scheme, the water film generates a higher $\mathrm{THz}$ electric field than the air does under the identical experimental condition. Unlike the case of air, $\mathrm{THz}$ wave generation from liquid water prefers a subpicosecond (200-800 fs) laser pulse rather than a femtosecond pulse ( $\sim 50 \mathrm{fs}$ ). This observation results from the plasma generation process in water.

For the two-color excitation scheme, the THz electric field is enhanced by one-order of magnitude in comparison with the one-color case. Meanwhile, coherent control of the $\mathrm{THz}$ field is achieved by adjusting the relative phase between the fundamental pulse and the second-harmonic pulse.

To eliminate the total internal reflection of $\mathrm{THz}$ waves at the water-air interface of a water film, a water line produced by a syringe needle was used to emit $\mathrm{THz}$ waves. As expected, more $\mathrm{THz}$ radiation can be coupled out and detected. As a supplement to the research of Terahertz Aqueous Photonics, $\mathrm{THz}$ wave generation from other liquids were also tested. For example, the $\mathrm{THz}$ electric field obtained from $\alpha$-Pinene is 1.8-times stronger than that from water under the same experimental condition, which mainly arises from the much weaker absorption of $\alpha$-Pinene for $\mathrm{THz}$ waves.

Historically liquid water has been sworn off as a THz source. This dissertation not only demonstrates that water can be used to generate $\mathrm{THz}$ waves but also contributes to enthralling insight into the development of intense $\mathrm{THz}$ sources. The work also indicates that $\mathrm{THz}$ waves can be a great tool for the investigation of laser-liquid interaction. 


\section{Contributors and Funding Sources}

This work was supported by a dissertation committee consisting of Professor Xi-Cheng Zhang (advisor) and Professor Wayne Knox from The Institute of Optics and Professor Roman Sobolewski from the Department of Electrical and Computer Engineering. The committee was chaired by Professor Yongli Gao from the Department of Physics and Astronomy at the University of Rochester.

All the experiments in the dissertation and simulations in Section 2.9 were performed in collaboration with Dr. Yiwen E. The data used to plot Figure 5.1 was also provided by Dr. Yiwen E. The experiments in Chapter 3 described in the dissertation were also done in collaboration with Professor Jianming Dai. Original setups and samples for water films were built by Kaia Williams. The experiment in Chapter 5 was performed jointly with Shenghan Gao. All other work conducted for the dissertation was completed by the author independently.

The research was primarily supported through grants of the National Science Foundation (ECCS-1916068), the Air Force Office of Scientific Re- 
search (FA9550-18-1-0357) and the Army Research Office (W911NF-16-10381, W911NF-16-1-0436, and W911NF-17-1-0428). 


\section{List of Figures}

1.1 Terahertz frequency band in the electromagnetic spectrum. Techniques used in electronics and photonics are both utilized to study terahertz waves. . . . . . . . . . . . . . . . . 2

1.2 A generic arrangement for a $\mathrm{THz}$ time-domain spectroscopy (THz-TDS) system. Pulses from the femtosecond laser are split at the beam splitter (BS). There is a delay stage used to alter the time of arrival at the $\mathrm{THz}$ receiver between the $\mathrm{THz}$ pulse and the laser pulse. . . . . . . . . . . . . . . . . 5

$1.3 \mathrm{THz}$ imaging of Badia Polyptych painted by Giotto. (a) Investigated area marked on the visible image. (b) Reflection $\mathrm{THz}$ image. (c) Non-invasive cross-section image of the artwork. (Reprinted with permission from [1], copyright 2010, Springer) 6

1.4 Experimental setup used for supercontinuum generation from water. (Reprinted with permission from [2], copyright 2003, Springer) . . . . . . . . . . . . . . . . . . . 14 
1.5 Spectra obtained from the propagation of a $170 \mathrm{fs}$ Ti: sapphire laser pulse in water at different input pulse energy: (a) 0.4$1.8 \mu \mathrm{J} ;$ (b) 2-200 $\mu \mathrm{J}$. (Reprinted with permission from [2], copyright 2003, Springer) . . . . . . . . . . . . . . . . . . . . . 15

1.6 Schematic setup for HHG from water. (Reprinted with permission from [3], copyright 2003, Springer)] . . . . . . . . . . . 17

1.7 Lower-order harmonic emission spectra detected for water. (Reprinted with permission from [3], copyright 2003, Springer) 18

1.8 Higher-order harmonic emission spectra detected for water. (Reprinted with permission from [3], copyright 2003, Springer) 19

1.9 The refractive index and power absorption of liquid water shown as a function of frequencies at four different temperatures. (Reprinted with permission from [4], copyright 1997, AIP

2.1 (a) Schematic diagram of a thin film flow system. UR, LR: upper, lower reservoir, AF: aluminum frame, DC: drop collector, PP: peristaltic pump. Tubes of lengths $\mathrm{L}_{1}, \mathrm{~L}_{2}, \mathrm{~L}_{3}$, and $\mathrm{L}_{4}$ connect the circuit. (b) Photograph of the water film. (Reprinted with permission from [5], copyright 2014, IEEE)] . . . . . . . . 24

2.2 Photo of the water film used in the experiment. Two aluminum wires are separated by $4 \mathrm{~mm}$. The thickness of the water film is controlled by the water flow rate. . . . . . . . . . . . . . . . 25 
2.3 Thickness of the water film versus the water flow rate. . . . 26

2.4 Experimental set-up for $\mathrm{THz}$ wave generation from a water film. Broadband THz wave is generated by tightly focusing the laser beam into a gravity-driven wire-guided free-flowing water film. The water film can be moved in the laser propagation direction by a mechanical translation stage. OAPM, off-axis parabolic mirror. HWP, half-wave plate. . . . . . . . . . . 28 
2.5 Measurements of the $\mathrm{THz}$ fields when the water film is translated along the direction of laser propagation. (a) THz waveforms are plotted from curve A to curve $\mathrm{C}$ when the water film is before, near, and after the focus, respectively; Curve B shows the $\mathrm{THz}$ waveform generated from liquid water; Curve $\mathrm{D}$ is the reference with no water film. Yellow spark and bluish pane represent the plasma and the water film respectively. THz emission angle shown in the figure is not meant to be indicative of actual $\mathrm{THz}$ emission pattern. (b) THz waveforms when the water film is moved near the focal point. The 0 position is set to the place with the strongest THz field. Relative positions are listed with the corresponding waveforms. The negative sign means the water film is located after the focal point. The positive sign indicates the opposite case. (c)

Comparison between the $\mathrm{THz}$ field from water and that from air plasma in the frequency domain. The dashed, solid, and dotted spectra correspond to curve A, curve B, and curve D in (a), respectively. The laser pulse is temporally stretched to $550 \mathrm{fs}$ for these measurements. . . . . . . . . . . . . . . . . . 30

2.6 Comparison of $\mathrm{THz}$ waves generated from water and air in the (a) time domain and (b) frequency domain. . . . . . . . . . 32 
2.7 The energy of $\mathrm{p}$-polarized $\mathrm{THz}$ field from liquid water with different linearly optical polarization. $0^{\circ}$ and $90^{\circ}$ refer to $\mathrm{p}-$ polarized and s-polarized optical beam respectively. . . . . . . 33

2.8 Normalized $\mathrm{THz}$ energy from liquid water as a function of incident optical pulse energy. The water film will be broken if the energy of the excitation pulse is over $420 \mu \mathrm{J}$. . . . . . . . 35

$2.9 \mathrm{THz}$ wave generation from water films with different thicknesses. The refractive index of water at $0.5 \mathrm{THz}$ is calculated to be 2.29 from the time shift of the $\mathrm{THz}$ field. The absorption coefficient of water at $0.5 \mathrm{THz}$ is calculated to be $146.2 \mathrm{~cm}^{-1}$ from the attenuation of the $\mathrm{THz}$ field's amplitude. . . . . . . . 36

2.10 Photo of a $120 \mu \mathrm{m}$ thick water film formed by a water jet with a flat nozzle. The laser beam is focused into the center of the film where is flat and stable. . . . . . . . . . . . . . . . . 37

2.11 Normalized THz energy as a function of the optical pulse energy. The red line shows a linear fit. . . . . . . . . . . . . . . . 38

$2.122 \mathrm{D}$ cross-section of the $\mathrm{THz}$ wave generation process in a water film. Intense pulses ionize water at the focal point in the direction of the refracted laser beam. The angle of incidence on the air-water interface is $\alpha$. The black arrow shows the dipole orientation direction. Due to the total internal reflection at the water-air interface, $\mathrm{THz}$ emission at $0.5 \mathrm{THz}$ can be coupled out only when $-24.6^{\circ}<\theta_{\mathrm{t}}<+24.6^{\circ}$. . . . . . . . . . . . . . 39 
2.13 Visualization diagram of laser-induced plasma formation. . . . 41

2.14 Illustration of incident angle $\alpha$ and angle of detection $\beta$. All angles are defined with respect to the $z$-axis. . . . . . . . . . . 45

$2.15 \mathrm{THz}$ waveforms generated from a water film with opposite angles of incidence $\left(\alpha= \pm 65^{\circ}\right)$. The corresponding spectra and dipole approximation illustration are shown in the insets. 46

2.16 Simulation result of normalized $\mathrm{THz}$ energy $I_{\mathrm{THz}}(\alpha, \beta)$ using the dipole radiation model. The dashed lines indicate the cases of $|\alpha-\beta|=90^{\circ}$, which means the detector is located in the plane of the water film. These dash lines separate the plots into three parts, labeled as "B", "F", and "B". "B" and "F" indicate backward and forward propagating THz signal, respectively. . 48

$2.17 \mathrm{THz}$ energy versus the angle of incidence $\alpha$ with $\beta=0^{\circ}$. The black squares are the data measured by EOS and the blue dots are measured by a Golay cell. Only forward propagating signals can be detected for $\beta=0^{\circ}$. . . . . . . . . . . . . . . . 49

$2.18 \mathrm{THz}$ energy versus the angle of incidence $\alpha$ with $\beta=55^{\circ}$. The blue dots are measured by a Golay cell. Backward propagating signals are detected for $-90^{\circ}<\alpha<-35^{\circ}$. . . . . . . . . . . . . 51 
2.19 Normalized $\mathrm{THz}$ energy from liquid water and air plasma with different pulse duration of the laser beam. Black squares represent the THz energy from liquid water and red dots represent the case of air plasma. The optical pulse duration is at its minimum of 58 fs when no frequency chirp is applied. On the left-hand side of the figure, negative chirps are applied to increase the optical pulse duration while the case of positive chirps is shown on the right-hand side of the figure. The energy of the laser pulse is $0.4 \mathrm{~mJ}$ for these measurements. . . 52

2.20 Spectra of $\mathrm{THz}$ radiation generated from the water film with a negative chirped 663 fs pulse and that with a positive chirped 647 fs pulse. . . . . . . . . . . . . . . . . . . . . . 53

3.1 Schematic diagram of $\mathrm{THz}$ wave generation from a two-color laser pulses induced air plasma. An intense femtosecond laser beam $\omega$ and its second harmonic $2 \omega$ are focused to generate plasma in the air. In the most common way, a $\beta$-BBO crystal is applied for the generation of $2 \omega$ pulse. The output $\mathrm{THz}$ waves are determined by the phase delay between $\omega$ pulse and $2 \omega$ pulse. . . . . . . . . . . . . . . . . . . 58 
3.2 Dependence of $\mathrm{THz}$ amplitude on the power of the (a) $\omega$ and (b) $2 \omega$ beams in $\mathrm{THz}$ wave generation by two-color laser

beams. (Reprinted with permission from [6], copyright 2006 American Physical Society) . . . . . . . . . . . . . 60

3.3 Schematic illustration of the experimental setup. Inside the dashed line is the inline phase compensator. $\beta$-BBO, beta barium borate crystal; BP, birefringent plate $(\alpha-\mathrm{BBO}) ; \mathrm{QW}$, quartz wedges; DWP, dual-wavelength wave plate; the upper and lower arrows near the laser beams indicate the polarization of the $\omega$ and $2 \omega$ beams, respectively. (b) A typical phase curve obtained by changing relative phase between the $\omega$ and $2 \omega$ pulses through the change of the insertion of one of the wedges while monitoring the $\mathrm{THz}$ average power with a pyroelectric detector when the $\omega$ and $2 \omega$ pulses are linearly polarized and parallel to each other; the inset shows a zoomed-in portion of the phase curve. (Reprinted with permission from [7], copyright 2009 American Physical Society)] . . . . . . . . 62

3.4 Schematic diagram of the experimental setup. A phase compensator composed of an $\alpha$-BBO crystal, a pair of wedges, and a dual-wavelength wave plate (DWP) is applied to control the relative phase between $\omega$ and $2 \omega$ pulses. PM, parabolic mirror with an effective focal length of 1-inch. . . . . . . . . . . . . . 64 
3.5 Comparison of $\mathrm{THz}$ waves generated from a $120 \mu \mathrm{m}$ thick water film with one-color and two-color excitation schemes. (a),

(b) Comparison in the case of a short optical pulse duration (58 fs) in the time domain and frequency domain, respectively. (c), (d) Comparison in the case of a long optical pulse duration (300 fs) in the time domain and frequency domain, respectively. Unified normalization ratios are labeled. . . . . . . 66

3.6 Modulation of $\mathrm{THz}$ wave generation from a water film. (a) Comparison of $\mathrm{THz}$ waveforms obtained when the relative phase between $\omega$ and $2 \omega$ pulses is changed by $\pi$ through the change of the insertion of one of the wedges in the phase compensator. Inset, $\mathrm{THz}$ electric field as a function of the phase delay between $\omega$ and $2 \omega$ pulses. (b) An overall phase scan for $\mathrm{THz}$ wave radiation from the water film obtained by gradually changing the phase between $\omega$ and $2 \omega$ pulses while monitoring the $\mathrm{THz}$ energy by using a Golay cell. The range of the phase delay is limited by the full length of the wedge. . . . . . . . . 68 
3.7 Normalized THz energy from liquid water as a function of the total excitation optical pulse ( $\omega$ and $2 \omega$ ) energy. Blue squares, $\mathrm{THz}$ energy calculated from the temporal integral of the $\mathrm{THz}$ waveform measured by EOS. Blue dots, modulated THz energy measured by the Golay cell. Red circles, unmodulated THz energy measured by the Golay cell. The maximum pulse energy is limited by the available laser pulse energy in the experiment. .................. . . 70

4.1 Photograph of syringe needles. The color of a syringe needle indicates its gauge number and inner diameter. . . . . . . . . 77

4.2 Photograph of the water line produced by a syringe needle with $260 \mu \mathrm{m}$ inner diameter. The diameter of the water line is $260 \mu \mathrm{m}$ as well. The flow velocity is about $7 \mathrm{~m} / \mathrm{s}$ along the $y$ direction. The laser beam propagates in the $z$-direction. The water line can be moved along the $x$-direction by a translation stage. . . . . . . . . . . . . . . . . . . . 78

4.3 (a) $\mathrm{THz}$ peak fields with different $x$ positions when the 260 $\mu \mathrm{m}$ diameter water line is crossing the laser focal point along the $x$-direction. (b) THz waveforms of $x= \pm 90 \mu \mathrm{m}$ in (a). . . 80 
4.4 Comparison of $\mathrm{THz}$ fields generated from a $210 \mu \mathrm{m}$ water line and a $120 \mu \mathrm{m}$ water film. EPL, effective path length. A shift of the $210 \mu \mathrm{m}$ water line in the $x$-direction makes the EPL to be $158 \mu \mathrm{m}$. Oblique incidence on the $120 \mu \mathrm{m}$ water film leads to a $151 \mu \mathrm{m}$ EPL. . . . . . . . . . . . . . . . . . . . . . . . . . 81

4.5 Effect of optical pulse duration on THz energy and peak electron density for a $210 \mu \mathrm{m}$ water line. The black dots are the experimental data for $\mathrm{THz}$ energy. The red curve is the simulation data for peak electron density. . . . . . . . . . . . . . . 83

4.6 Optimal optical pulse duration versus the diameter of the water line. The blue squares are simulations of optimal pulse duration aiming for the highest electron density. The red dots are the experimental data of optimal pulse duration obtained with the strongest $\mathrm{THz}$ energy. . . . . . . . . . . . . . . . . . 86

4.7 THz energy versus the diameter of the water line. . . . . . . . 88

5.1 Waveform measurement of $\alpha$-Pinene as the sample by using a standard THz-TDS system. The black curve is the reference waveform with no sample presented. . . . . . . . . . . . . . . 92

5.2 Measured results of refractive index and field absorption coefficient of $\alpha$-Pinene in the frequency region $0.5-2.5 \mathrm{THz} . \quad$. . . . 93 
5.3 Comparison of $\mathrm{THz}$ waves generated from $\alpha$-Pinene and water in (a) time domain and (b) frequency domain. The diameter of the liquid line is $210 \mu \mathrm{m}$. Laser pulse energy is $0.4 \mathrm{~mJ}$. Optical pulse durations are individually optimized for $\alpha$-Pinene and water. Both have a value around 345 fs. The dash line in (b) is calculated by removing the absorption of $\alpha$-Pinene and adding the absorption of water to the black curve from 0.5

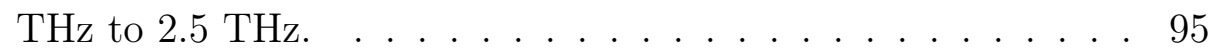




\section{Chapter 1}

\section{Introduction}

In this chapter, background information is provided for the investigation of Terahertz Aqueous Photonics. Unique properties and applications of terahertz waves are presented. Then three extensively used pulsed terahertz sources are reviewed: photoconductive antenna, nonlinear crystal, and gas. Knowing the success of using water to emit electromagnetic waves in other frequency regions, it is expected that liquid water can also be acted as a terahertz source. Potential challenges and opportunities are discussed as well.

\subsection{Terahertz Frequency Band}

The International Telecommunication Union designated the electromagnetic waves with the band of frequencies from $0.3 \times 10^{12} \mathrm{~Hz}$ to $3 \times 10^{12} \mathrm{~Hz}$ as terahertz radiation. Another common definition of terahertz frequency ranges from $0.1 \times 10^{12} \mathrm{~Hz}$ to $10 \times 10^{12} \mathrm{~Hz}$. Figure 1.1 shows the terahertz frequency band in the electromagnetic spectrum [8]. In the spectrum, terahertz waves 
lie between microwaves and infrared waves. Due to the absence of efficient terahertz emitters and sensitive terahertz detectors before the 1980s, terahertz frequency band was considered to be an unexplored realm. By contrast, microwaves in electronics and infrared waves in photonics has been successful applied in sensing, communication, heating, and spectroscopy. With the development of high-speed optoelectronics techniques, semiconductors, and ultrafast lasers, a flourish of the terahertz community comes.

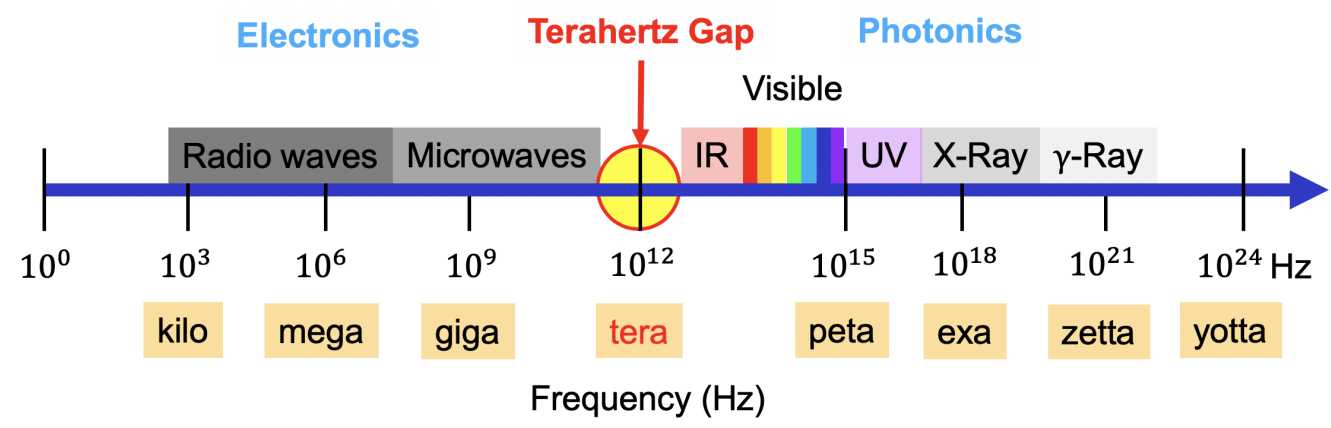

Figure 1.1: Terahertz frequency band in the electromagnetic spectrum. Techniques used in electronics and photonics are both utilized to study terahertz waves.

One terahertz is $10^{12} \mathrm{~Hz}$ or $1 \mathrm{THz}$. In ensuing sections, "THz" will be used as an abbreviation of "terahertz" for simplicity. To have a better understanding of $\mathrm{THz}$ waves, it is essential to take a look at how " $1 \mathrm{THz}$ " is expressed in different units. Common parameters corresponding to an oscillation frequency of $1 \mathrm{THz}$ are listed as follows [8]:

- Frequency: $\nu=1 \mathrm{THz}$;

- Period of oscillation: $\tau=1 / \nu=1 \mathrm{ps}$; 
- Wavelength: $\lambda=c / \nu=300 \mu \mathrm{m}$;

- Photon energy: $h \nu=4.1 \mathrm{meV}$;

- Equivalent temperature: $T=h \nu / k_{\mathrm{B}}=48 \mathrm{~K}$.

$c$ is the speed of light in vacuum, $h$ is the Plank constant, and $k_{\mathrm{B}}$ is the Boltzmann constant.

\subsection{Properties and Applications}

Knowing the parameters that are related to electromagnetic waves with an oscillation frequency of $1 \mathrm{THz}$, unique properties and potential applications of $\mathrm{THz}$ waves can be unveiled.

Many molecules have spectral features in the THz frequency band due to absorption and scattering caused by molecular oscillation and rotation. Thus, identifying the composition of a material is an important application for THz spectroscopy. One popular technique developed for this purpose is called THz time-domain spectroscopy (THz-TDS) [9]. Figure 1.2 shows a typical arrangement of a THz-TDS system. A laser beam is split into two parts by a beam splitter (BS). The pump beam is used to excite the $\mathrm{THz}$ emitter to generate $\mathrm{THz}$ radiation while the probe beam detects the signal that is proportional to the electric field of the $\mathrm{THz}$ pulse. The time delay between the two beams is controlled by a mechanical translation stage. The measurement of $\mathrm{THz}$ waveforms happens when the generated $\mathrm{THz}$ pulse and the probe pulse both reach the receiver. Since the laser pulse oscillates much 
faster than the $\mathrm{THz}$ pulse does, the receiver can be regarded as an ultrafast gated detector. The probe laser pulse with the gated nature samples the waveform of the $\mathrm{THz}$ electric field. When the unidentified sample is placed in the THz beam path, the THz signal that carries the sample's information will be sensed. In order to obtain the true contribution from the sample, a reference signal with no sample presented has to be recorded. Compared to the reference signal, the signal from the sample usually has a reduced amplitude, a time shift, and oscillations in its tail. The reduced amplitude results from the absorption for $\mathrm{THz}$ waves. The time shift indicates that the $\mathrm{THz}$ waves pass through a material with a refractive index greater than the index of refraction of air. The oscillations have the feature of resonant absorption.

The timescale of $\mathrm{THz}$ waves is around one picosecond, making it a great tool in pump-probe experiments to study ultrafast phenomena such as electron oscillation and molecular rotation [10].

The wavelength of $\mathrm{THz}$ waves ranges from hundreds of microns to a few millimeters. The wavelength of an electromagnetic wave determines its interaction with a physical structure. Absorption and scattering will occur if the wavelength is comparable with the dimension of the structure. Otherwise, there will be no substantial interaction. The long wavelength of $\mathrm{THz}$ waves leads to little attenuation when $\mathrm{THz}$ waves pass through many dielectric materials, such as paper, wood, plastic, clothing, and glass. Therefore, $\mathrm{THz}$ waves can be used for the imaging of opaque objects [11, 12]. In addition, 


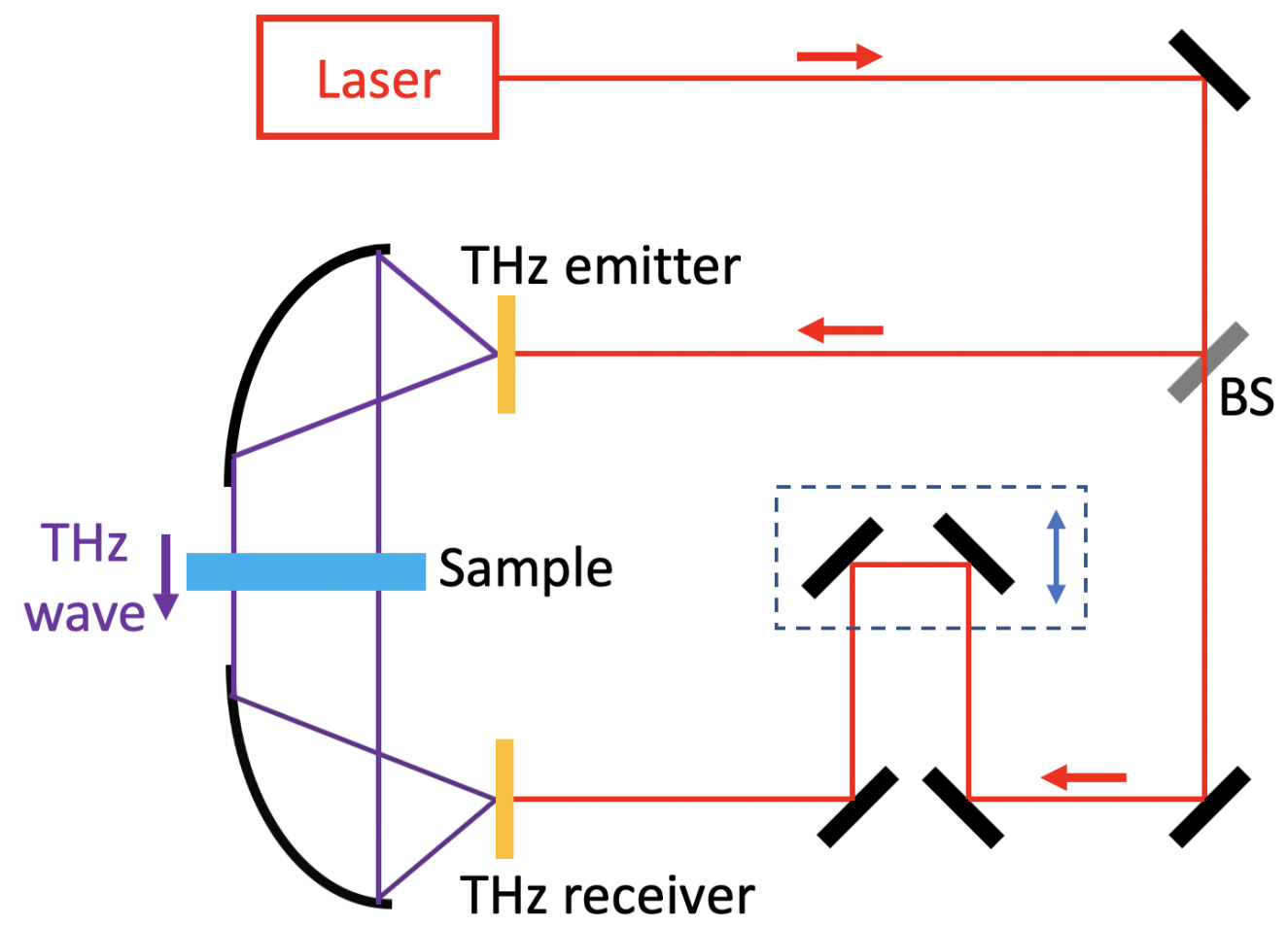

Figure 1.2: A generic arrangement for a $\mathrm{THz}$ time-domain spectroscopy (THz-TDS) system. Pulses from the femtosecond laser are split at the beam splitter (BS). There is a delay stage used to alter the time of arrival at the $\mathrm{THz}$ receiver between the $\mathrm{THz}$ pulse and the laser pulse. 
$\mathrm{THz}$ radiation is the ideal source for imaging in some poor environmental conditions (such as dense smoke and dust). Figure 1.3 shows an example of THz imaging [1]. The image reveals high reflection values of the gold leaf. The presence of gold leaves beneath the paint layers can be clearly observed around the outline of the head and wings of the angel.

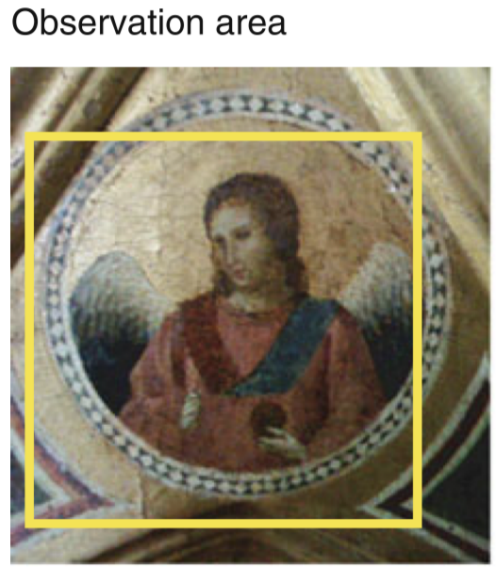

a
$\mathrm{THz}$ image

Gold foil under pigments

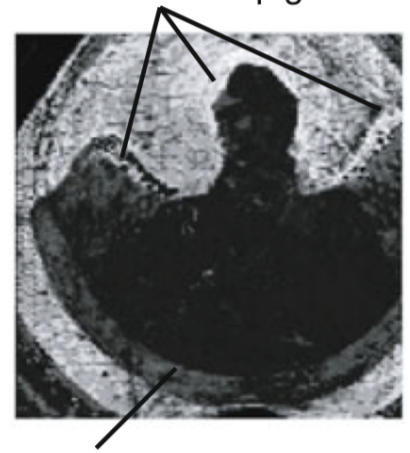

Lead white

b

Non-invasive cross section image

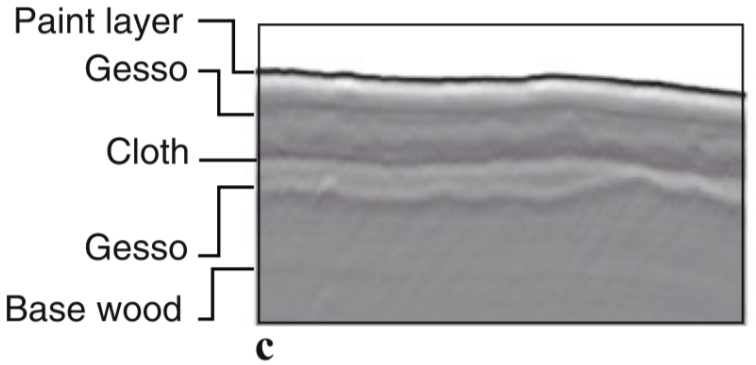

Figure 1.3: THz imaging of Badia Polyptych painted by Giotto. (a) Investigated area marked on the visible image. (b) Reflection $\mathrm{THz}$ image. (c) Non-invasive cross-section image of the artwork. (Reprinted with permission from [1], copyright 2010, Springer) 
A $\mathrm{THz}$ photon has an energy level of meV. This photon energy is lower than the energy of chemical bonds, assuring that $\mathrm{THz}$ radiation induces no destruction from the interaction. As a comparison, the photon energy of X-rays ranges from $100 \mathrm{eV}$ to $100 \mathrm{keV}$, which can be harmful to biomedical samples and human beings. The safety of $\mathrm{THz}$ radiation is a requisite for its applications in nondestructive evaluation and safety check.

\subsection{Pulsed $\mathrm{THz}$ sources}

Efficient, broadband, and robust $\mathrm{THz}$ sources are essential for the above applications. This section focuses on introducing different types of popular pulsed THz sources, including photoconductive antennae, nonlinear crystals, and gases.

\subsubsection{Photoconductive Antenna}

A photoconductive antenna (PCA) is also known as a photoconductive switch or an Auston switch. It is one of the most widely used devices for $\mathrm{THz}$ wave generation and detection. A PCA is usually composed of two metal electrodes deposited on a semiconductor substrate (such as low-temperature grown GaAs [13, 14]). If a femtosecond optical pulse with photon energy greater than the bandgap of the semiconductor is focused onto the gap between the two electrons, electron-hole pairs will be produced. The density

of these charged particles will rapidly increase to "turn on" the switch. Free 
carriers are then accelerated by the electric field generated from an applied DC voltage between the two electrodes. Occurred transient photocurrents in this process result in the radiation of electromagnetic waves. When the PCA is switched by a femtosecond pulse, the transient current $J$ has a picosecond time-scale leading to the emission of a $\mathrm{THz}$ pulse with an electric field $E_{\mathrm{THz}}$, as described by the following expression [15]:

$$
E_{\mathrm{THz}} \propto \partial J(t) / \partial t
$$

$\mathrm{THz}$ wave generation from a PCA is the most popular approach used in commercial $\mathrm{THz}$ products because it does not require intense optical pulses for excitation. As a compromise, a high external voltage is a requisite to build the electric field between the electrodes for the generation of $\mathrm{THz}$ pulses. Besides the possibility of causing a breakdown in the substrate, a high bias voltage will lead to a large amount of electric noise that harms the system.

\subsubsection{Nonlinear Crystal}

Nonlinear crystals can emit $\mathrm{THz}$ waves through optical rectification, which is a second-order nonlinear effect. It can be understood as a reverse process of electro-optic (EO) effect. When an intense femtosecond optical pulse passes through a nonlinear material, a static polarization is produced. The induced polarization is proportional to the envelope of the optical pulse. The timedependent optical field results in the time-varying polarization, which acts 
as the source for $\mathrm{THz}$ radiation.

Since a second-order nonlinear effect only occurs in a noncentrosymmetric material, $\mathrm{ZnTe}, \mathrm{GaAs}, \mathrm{GaP}, \mathrm{InP}, \mathrm{LiNbO}_{3}$, and $\mathrm{LiTaO}_{3}$ are utilized to generate $\mathrm{THz}$ waves through optical rectification. In such a nonlinear process, phase matching [16] is a crucial factor in deciding the $\mathrm{THz}$ emission. The phase matching condition requires that the group velocity at optical frequencies matches the phase velocity at $\mathrm{THz}$ frequencies:

$$
v_{\text {group }, \text { optical }}=v_{\text {phase }, \mathrm{THz}}
$$

With appropriate choice of nonlinear crystal and wavelength of the optical pulse, this condition can be satisfied within a certain spectral regime. One of the most successful examples is ZnTe, which satisfies the criteria when 800 nm central wavelength optical pulses (Ti: sapphire laser) are available.

Some other crystals such as $\mathrm{LiNbO}_{3}$ have a larger EO coefficient than ZnTe does. However, there is a significant mismatch between the group velocity of optical pulses and the phase velocity of $\mathrm{THz}$ pulses in $\mathrm{LiNbO}_{3}$ with a colinear geometry. To overcome the issue of mismatch but still benefit from the large $\mathrm{EO}$ coefficient of $\mathrm{LiNbO}_{3}$, a method of tilting the optical pulse front was proposed by Helbing et al. [17, 18, 19. Moreover, organic crystals (DUST, DSTMS and OH1) that exhibit high EO coefficients have also been demonstrated as intense $\mathrm{THz}$ sources even though they are very delicate under intense optical excitation [20, 21, 22]. 


\subsubsection{Gas}

Compared with PCA or nonlinear crystal, gas is capable of emitting $\mathrm{THz}$ waves even if very intense optical pulses are applied. These optical pulses ionize the gas to form plasmas (electrons and ions), which radiates $\mathrm{THz}$ pulses. The ambient air is the most commonly used one in this type of $\mathrm{THz}$ sources.

There are various approaches to achieve $\mathrm{THz}$ wave generation from air plasma. In the first demonstration by Hamster et al. in 1993, optical pulses with a single frequency were used [23]. The THz radiation is attributed to the ponderomotive force-induced acceleration of electrons in the plasma [23, 24]. Although the generation efficiency of this method is not great, the THz radiation can be scaled up by increasing the power of pump pulses without the worry of causing damage to the source. More recently, the generation efficiency was proved to be several orders of magnitude higher when a two-color excitation technique was used [25]. Furthermore, water vapor was reported emitting strong $\mathrm{THz}$ waves by using this method as well [26]. The THz wave generation process in this scheme has been explained by a four-wave mixing model [6, 25], a transient photocurrent model [27, 28, 29], or a full quantum model [30]. More details about the two-color excitation scheme will be discussed in Chapter 3. 


\subsubsection{Magnetic Heterostructure}

$\mathrm{THz}$ wave generation from magnetic heterostructures is a relatively new topic. It was demonstrated by Kampfrath et al. in 2013 [31]. The generation mechanism can be described as follows. A laser pulse is used to drive spins from a 10-nm-thin ferromagnetic iron film into a 2-nm-thin non-magnetic cap layer (ruthenium or gold). This resulting longitudinal spin current is converted into a transverse charge current $j_{c}$ in the $z$-direction by the inverse spin-Hall effect. This spin current acts as the source of $\mathrm{THz}$ radiation. The THz field $E$ satisfies the following equation:

$$
E(\omega)=Z(\omega) e \int_{0}^{d} \mathrm{~d} z j_{c}(z, \omega),
$$

where $Z(\omega)$ is the impedence, $e$ is the electron charge, $d$ is the film thickness.

In 2016, the same group reported a thorough investigation into this metallic spintronic $\mathrm{THz}$ emitter [32]. By using a 5.8-nm-thick $\mathrm{W} / \mathrm{CoFeB} / \mathrm{Pt}$ trilayer, $\mathrm{THz}$ waves have peak fields of several $100 \mathrm{kV} / \mathrm{cm}$ with the bandwidth covering the 1-30 THz range were produced. The generated field strength and bandwidth are impressive when considering that the pump source is a lowpower femtosecond laser oscillator. Even though the spintronic THz source outperforms other laser-oscillator-driven sources in terms of field strength and bandwidth [32], it is worth mentioning that some nonlinear crystals $\left(\mathrm{LiNbO}_{3}\right.$ or organic crystals) are capable of producing much stronger $\mathrm{THz}$ fields $(\sim \mathrm{MV} / \mathrm{cm})$ if an amplified laser is used. 


\subsection{Success of Using Liquid Water as the Source in Other Frequency Regions}

It is noticeable that liquid sources were not mentioned in the last section. In fact, there is no demonstration of $\mathrm{THz}$ wave generation from liquids before 2017. Especially, liquid water has been historically considered as a "foe" in the $\mathrm{THz}$ community due to its significant absorption in the $\mathrm{THz}$ frequency range [4, 33. In contrast, people have successfully used liquid water to generate electromagnetic waves in other frequency regions for years.

Water is involved in a variety of physical and chemical processes on the earth. It exhibits complicated structures and abnormal properties in different

conditions. For example, the temperature dependence of the density of water is not linear and the density rises to a peak at $3.98{ }^{\circ} \mathrm{C}$ [34]. Besides, water has strong cohesion, adhesion, and surface tension. Thus, water can be used to form a thin film. Transparent, stable, nontoxic, and inodorous water is the ideal target in physics, biology, and chemistry. Considering its accessibility and plenty, water has the potential to be the low-cost source for various electromagnetic waves.

Water vapor was reported as a source (water maser) emitting microwaves at 22.0 GHz as early as 1986 [35]. Maser stands for "microwave amplification by stimulated emission of radiation". The basic principle of maser is stimulated emission. Coherent radiation is produced by placing the amplifying medium into a resonant cavity. Maser-like stimulated emission has also been 
observed in nature from interstellar space. Such emission was observed from water molecules in 1986 [35]. It was found that water molecules in starforming regions could undergo a population inversion and emit radiation at about $22.0 \mathrm{GHz}$ [36]. In 1994, water maser emission from X-ray-heated circumnuclear gas was reported by the same group [37.

The laser works in a similar principle as the maser does but produces coherent radiation with higher frequency (usually at visible or infrared wavelengths). Benefiting from the rapid development of laser technology, researchers are capable of studying interactions between high-intensity laser and matter. In recent years, supercontinuum emission and extreme ultraviolet (XUV) generation from liquid water through nonlinear processes have been successfully observed as well.

\subsubsection{Supercontinuum Generation from Water}

In 1970, supercontinuum generation was discovered by Robert Alfano [38]. Supercontinuum generation is a process wherein laser lights with a narrow spectral bandwidth are converted into lights with a very broad spectral bandwidth. Supercontinuum is sometimes called white-light that usually refers to the radiation with spectra across 400-1100 nm. In 1987, the first observation of supercontinuum generation from water was reported [39]. When the incident laser pulse is of femtosecond duration, the spectrally broadened light emerges as a white disk surrounded by a rainbow-like pattern.

In 2003, experimental and theoretical results of white-light generation 


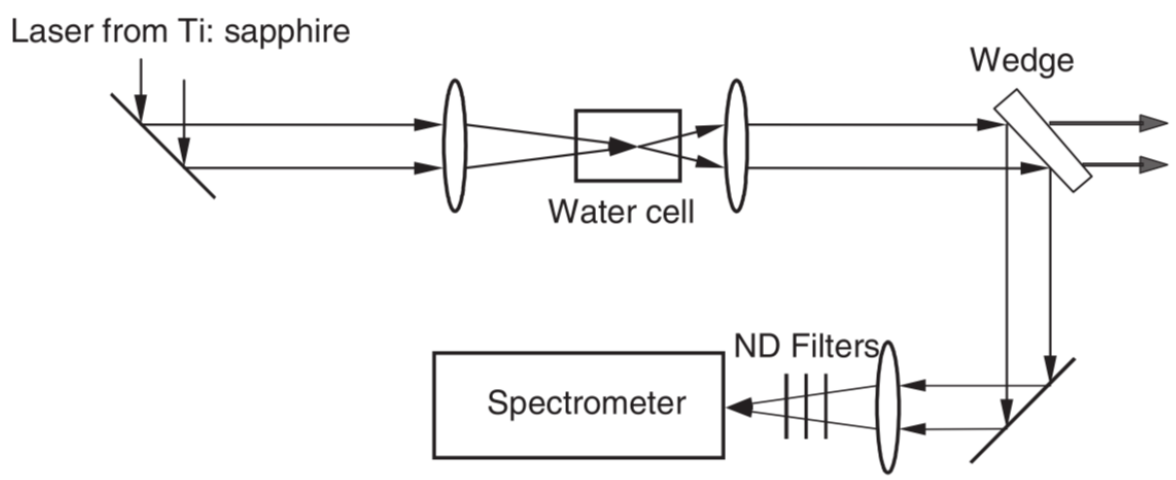

Figure 1.4: Experimental setup used for supercontinuum generation from water. (Reprinted with permission from [2], copyright 2003, Springer)

from the filamentation in water were presented [2]. Figure 1.4 shows the experimental setup for the supercontinuum generation from water. A $1 \mathrm{kHz}$ laser beam with the pulse energy of $2 \mathrm{~mJ}$ was used. The laser pulses were focused by a microscope objective into a water cell. The transmitted spectrum was collimated by an achromatic lens and imaged into the spectrometer. The measurement result is shown in Figure 1.5 .

The mechanisms for supercontinuum generation from water were fully discussed in 2]. Supercontinuum generation is the result of combined dynamic processes [2], such as self-focusing, group velocity dispersion (GVD), intensity clamping [40], self-steepening, anti-Stokes spectral broadening, filament fusion, filament breakup, and filament competition. Due to the nonlinear transformation of the laser pulse propagation in the water, the continuum spectrum of all frequencies arises.

In 2005, a systematical study of the influence of different parameters on 


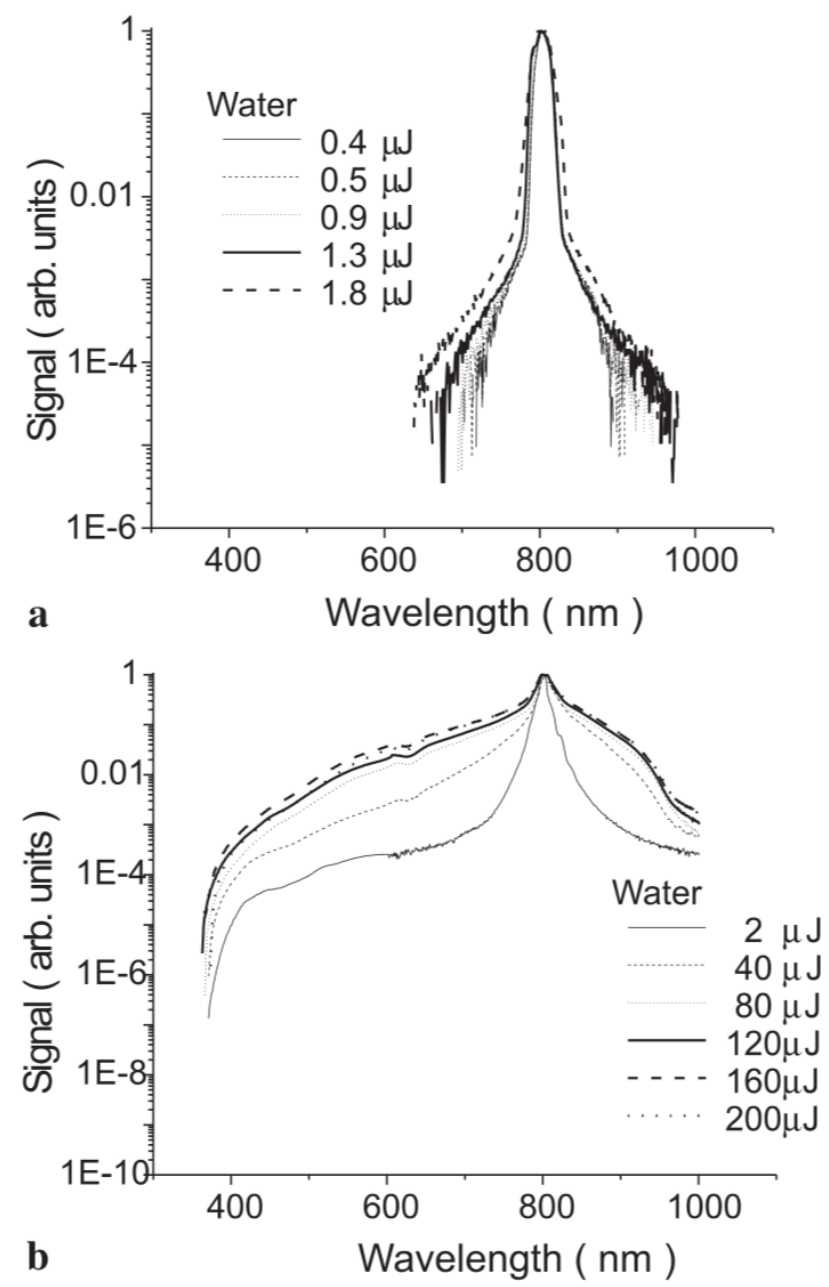

Figure 1.5: Spectra obtained from the propagation of a 170 fs Ti: sapphire laser pulse in water at different input pulse energy: (a) 0.4-1.8 $\mu \mathrm{J}$; (b) 2-200 $\mu \mathrm{J}$. (Reprinted with permission from [2], copyright 2003, Springer) 
the white-light generation was reported [41]. This study emphasized the focal position of the incident laser light within the medium and the pulse duration of the incident laser beam. Later, white-light generation with phase discontinuities and steeper intensity gradients was achieved with a 1-D spatial light modulator [42]. In 2016, the output supercontinuum spectra were extended to $2000 \mathrm{~nm}$, by choosing a pump wavelength at $1860 \mathrm{~nm}$ [43]. No visible radiation was detected in this measurement.

\subsubsection{XUV Generation from Water}

XUV light has numerous applications in photoelectron spectroscopy and lithography 44. Water has been found to be an efficient media to convert visible laser radiation into XUV [45, 46], wherein high-order harmonic generation (HHG) from atoms and molecules is an established source for XUV radiation [47]. In 2003, HHG from water microdroplets was first reported [3].

In this nonlinear process, laser radiation is converted into integer multiples of its fundamental frequency up to very high orders. This phenomenon occurs when intense ultrashort laser pulses interact with a dielectric medium. Initially bound electrons are field-ionized at particular phases of the laser electric field (close to its peak) and are driven away from their parent ions. Once the electric field of the laser pulse reverses, the electrons will decelerate on their outward-bound path and accelerate back towards their parent ions. Some of the ionized electrons will finally return to the ion core and recombine with the parent ions. During the time of recombination, the electrons 


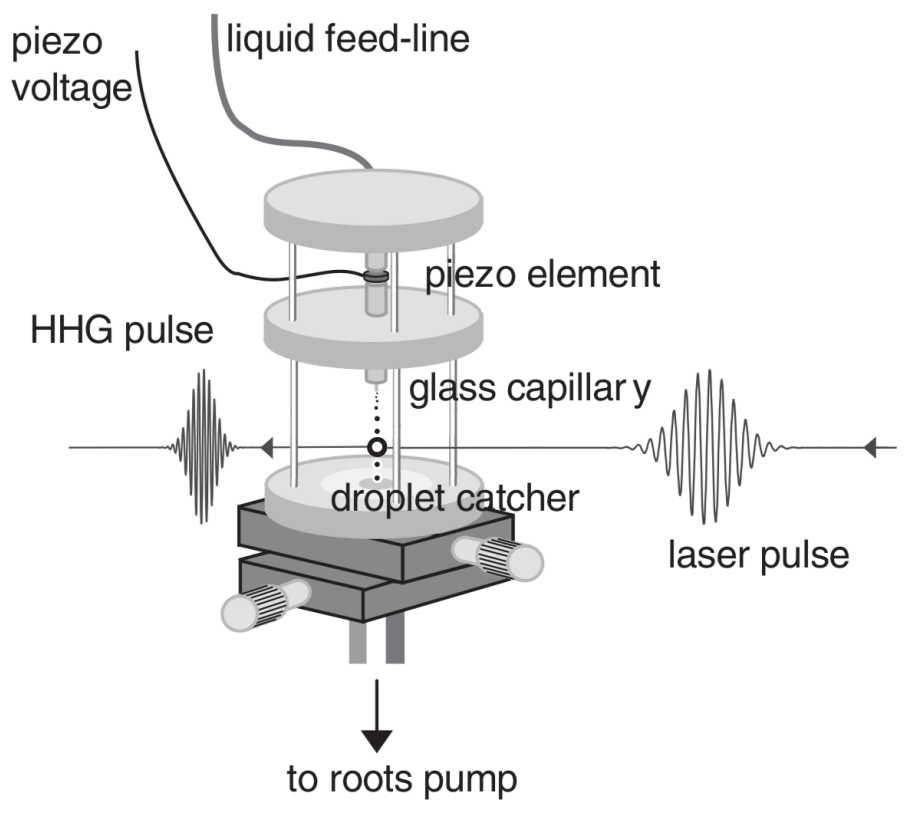

Figure 1.6: Schematic setup for HHG from water. (Reprinted with permission from [3], copyright 2003, Springer) 
generally possess nonzero kinetic energy. One photon per electron is emitted carrying the sum of the electrons kinetic energy plus the ionization potential $I_{\mathrm{P}}$. Classical and quantum calculations show that the maximum photon energy to be emitted in this process scales as [3]:

$$
\hbar \omega=I_{\mathrm{P}}+3.17 U_{\mathrm{P}},
$$

where $U_{\mathrm{P}}$ represents the ponderomotive potential from the laser field.

Figure 1.6 shows the schematic setup for HHG from water microdroplets. The glass capillary was mounted inside a vacuum chamber and the water droplet jet was aimed vertically downwards. A piezo element was used to control the droplet's repetition rate. Optical pulses interact with the water droplet to induce HHG. Corresponding measurements of HHG are shown in Figure 1.7 and Figure 1.8 [3].
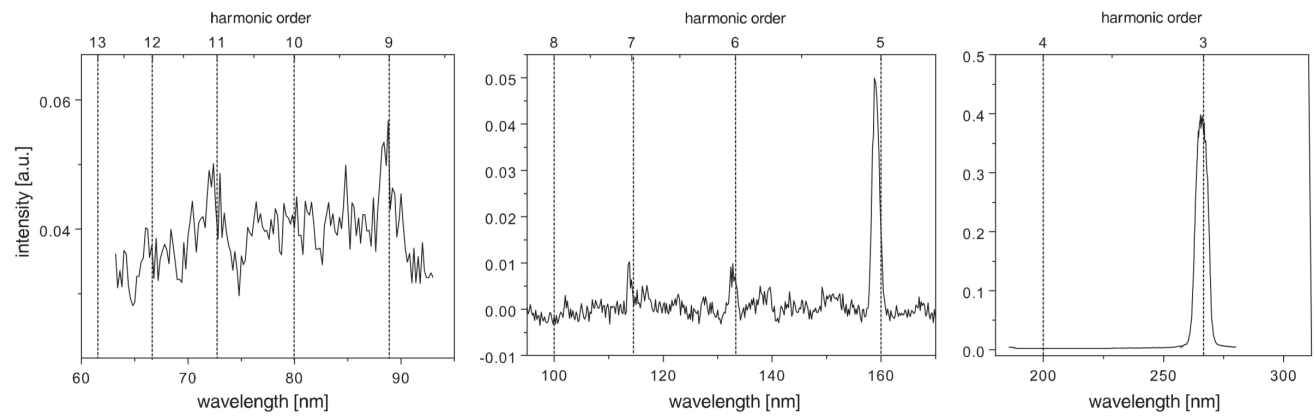

Figure 1.7: Lower-order harmonic emission spectra detected for water. (Reprinted with permission from [3], copyright 2003, Springer) 


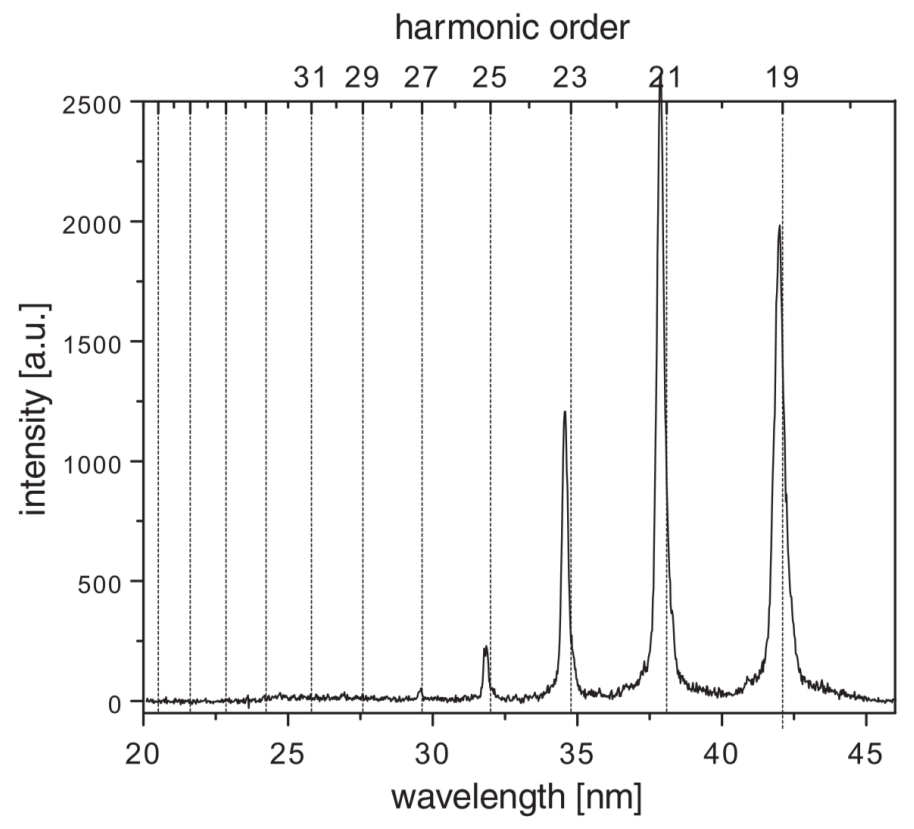

Figure 1.8: Higher-order harmonic emission spectra detected for water. (Reprinted with permission from [3], copyright 2003, Springer) 


\subsection{Challenges and Opportunities}

As discussed in the last section, water has been used to emit electromagnetic waves from XUV to infrared waves under the excitation of intense laser pulses. A similar approach of focusing intense optical pulses to excite water may also work for the generation of $\mathrm{THz}$ waves, which may enable the investigation of Terahertz Aqueous Photonics.

However, there are more challenges in obtaining $\mathrm{THz}$ radiation from water. The first problem leading the impediment is that liquid water has strong absorption characteristics in the $\mathrm{THz}$ frequency regime. Its power absorption at $\mathrm{THz}$ frequencies was measured by Keiding et al. in 1997. The result is shown in Figure 1.9 [4]. The power absorption coefficient of $220 \mathrm{~cm}^{-1}$ at $1 \mathrm{THz}$ means only one photon at $1 \mathrm{THz}$ can go through $1 \mathrm{~mm}$ thick water layer with $3.6 \times 10^{9} \mathrm{THz}$ photons entered at room temperature. Thus, $\mathrm{THz}$ signals may be completely buried in the absorption even if $\mathrm{THz}$ radiation has already been generated from water. Moreover, unlike the case in air, laser-induced breakdown and ionization process in water have not been well studied. Optimal conditions need to be experimentally tested. Refraction and total internal reflection caused by the interfaces between water and air make the situation more complicated.

Opportunities can also be seen in the Terahertz Aqueous Photonics. Compared with air, liquid water has a lower ionization threshold but a higher

molecular density [48, 49, 50, 51], which indicates that under the identical 


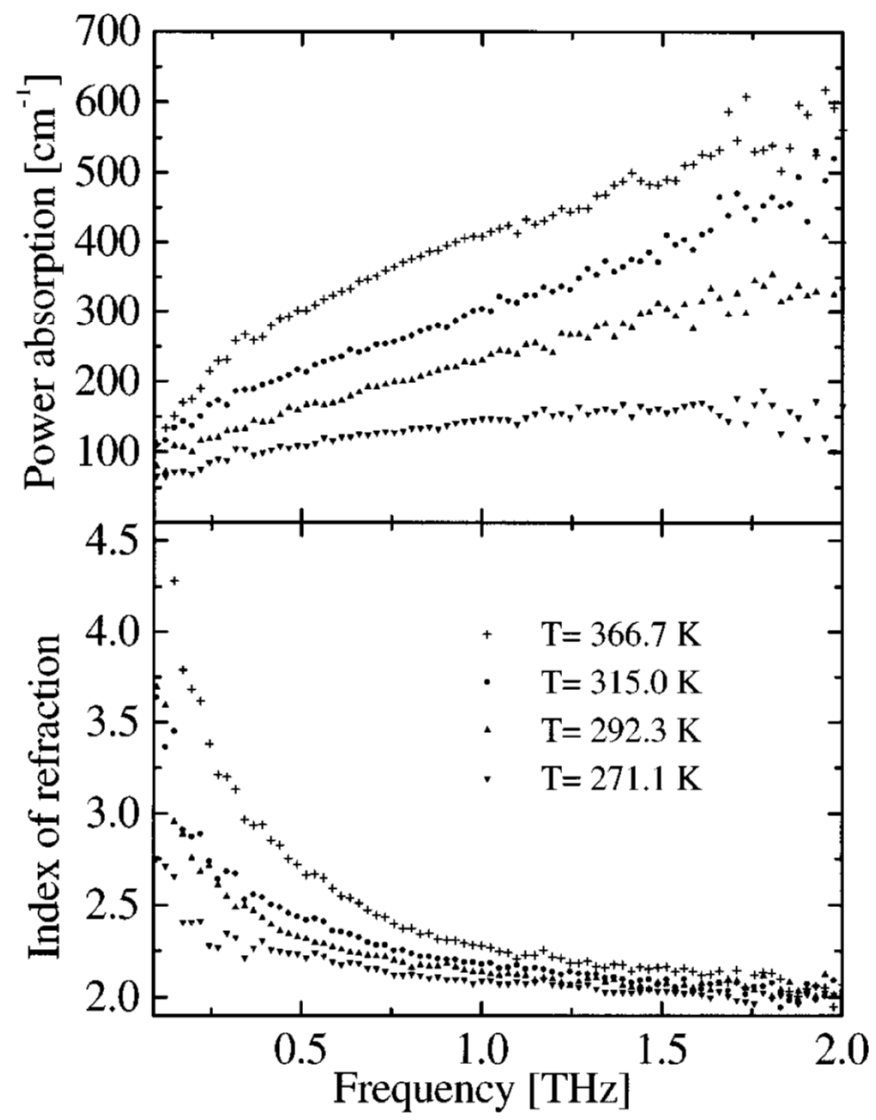

Figure 1.9: The refractive index and power absorption of liquid water shown as a function of frequencies at four different temperatures. (Reprinted with permission from [4], copyright 1997, AIP) 
excitation liquid water can provide more electrons and ions in the same unit volume. Unlike PCA and nonlinear crystals that suffered from permanent damage under the excitation of intense laser pulses [21, 52, 53], liquid water can quickly replenish itself due to its fluidity. Hence, liquid water has the potential to be developed as a $\mathrm{THz}$ source to meet desired applications. In addition, $\mathrm{THz}$ waves generated from liquid water will be closely related to laser-induced ionization process in water. Therefore, Terahertz Aqueous Photonics could provide more in-depth information on laser-water interaction. 


\section{Chapter 2}

\section{Terahertz Wave Generation from a Wa- ter Film}

The focus of this chapter is the demonstration of $\mathrm{THz}$ wave generation from liquid water. A thin water film is introduced as the source for $\mathrm{THz}$ radiation. The experimental demonstration of broadband $\mathrm{THz}$ waves emitted from the water film is shown. Dependence of the $\mathrm{THz}$ radiation upon optical polarization, pulse energy, pulse duration, and the water film's thickness is individually investigated as well. Laser-induced plasma formation associated with a ponderomotive force-induced dipole approximation radiation model is

proposed to explain the generation process. Corresponding simulations and experimental observations are also presented.

\subsection{Free-Flowing Thin Water Film}

Bulk liquid water is a strong absorber in the $\mathrm{THz}$ frequency range, making liquid water has historically been sworn off as a $\mathrm{THz}$ source. To mitigate 
the considerable loss of $\mathrm{THz}$ waves, the water with much less than $1 \mathrm{~mm}$ thickness is an intuitive choice to study $\mathrm{THz}$ wave generation.
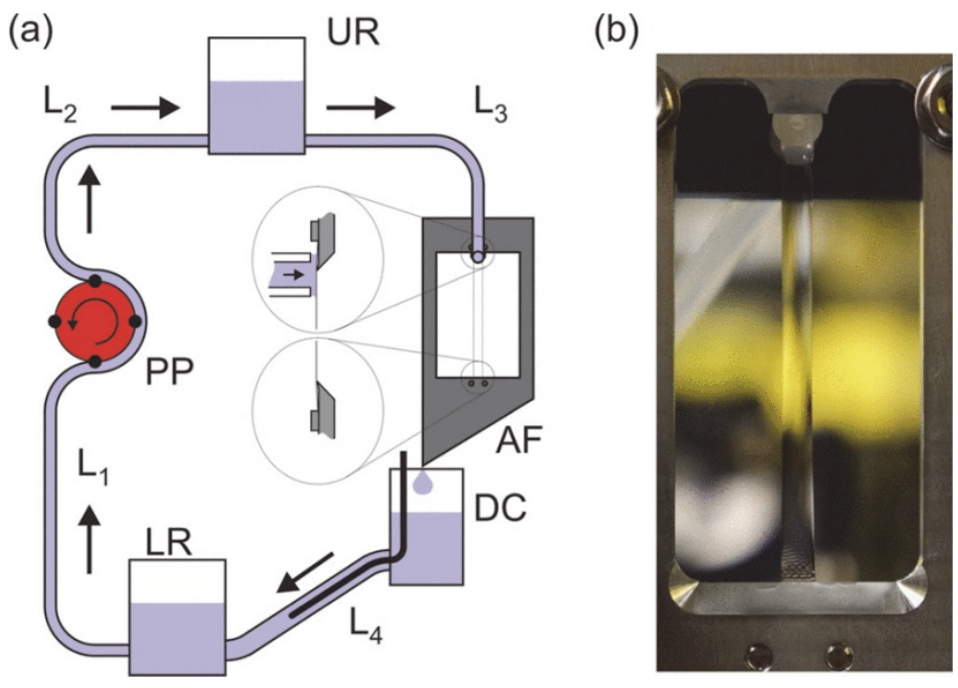

Figure 2.1: (a) Schematic diagram of a thin film flow system. UR, LR: upper, lower reservoir, AF: aluminum frame, DC: drop collector, PP: peristaltic pump. Tubes of lengths $\mathrm{L}_{1}, \mathrm{~L}_{2}, \mathrm{~L}_{3}$, and $\mathrm{L}_{4}$ connect the circuit. (b) Photograph of the water film. (Reprinted with permission from [5], copyright 2014, IEEE)

Recently, gravity-driven, free-flowing water films have been efficaciously used owing to their simple design and almost unmatched ability to generate a thin, continuous, and stable film of liquid water in free space [5]. Figure 2.1(a) shows the schematic diagram of the thin water film system in [5]. The gravity-assisted flow along two $18 \mu \mathrm{m}$ diameter metal wires forms the water film as shown in Figure 2.1(b). The thickness of the water film is adjusted by the height difference between the upper reservoir (UR) and the top of the wires mounted in an aluminum frame $(\mathrm{AF})$. The system is circulated by a 
peristaltic pump (PP).

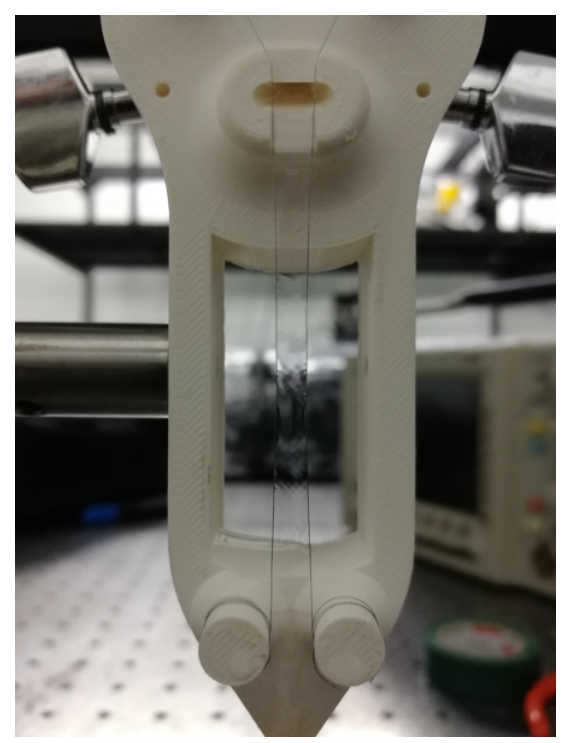

Figure 2.2: Photo of the water film used in the experiment. Two aluminum wires are separated by $4 \mathrm{~mm}$. The thickness of the water film is controlled by the water flow rate.

By using the concept of water flowing along wires, a similar water film system was built. These wires have a diameter of $170 \mu \mathrm{m}$, and are separated by $4 \mathrm{~mm}$. Under the effect of water's surface tension, the water goes along the wires and forms a water film as shown in Figure 2.2. Unlike the case in [5], the thickness of this water film was adjusted by throttling the water flow rate. An optical second-harmonic intensity autocorrelator was used to measure and calibrate the thickness of the water film. The corresponding result is plotted in Figure 2.3, which shows a linear relationship between the thickness of the water film and the flow rate. In this system, the thickness 
of the water film can be varied from $50 \mu \mathrm{m}$ to $330 \mu \mathrm{m}$.

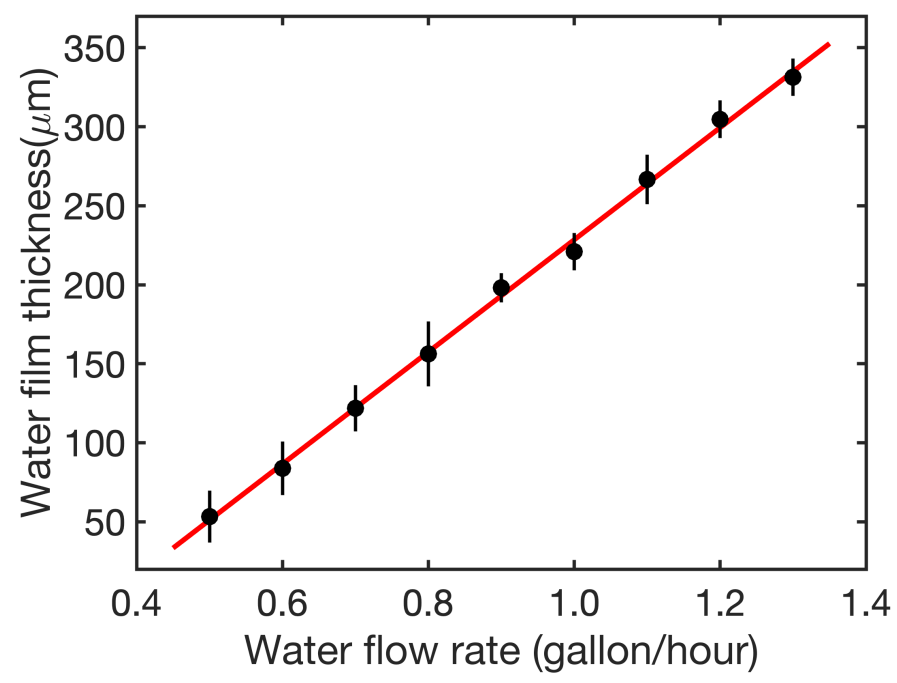

Figure 2.3: Thickness of the water film versus the water flow rate.

\subsection{Experimental Set-up}

Figure 2.4 schematically shows the experimental set-up for the generation of THz waves. An amplifier laser (Ti: sapphire) with $800 \mathrm{~nm}$ central wavelength and $1 \mathrm{kHz}$ repetition rate was used. An optical polarizer was placed to assure the p-polarized optical beam. The following half-wave plate (HWP) further adjusted the polarization of the optical beam. The incident angle of the laser beam on the water film was tilted to $25^{\circ}$ from the normal to reduce the water sputtering onto the surface of optics. The thickness of the water film was set to be $177 \mu \mathrm{m}$. The laser beam was focused into the water film by 
a 1-inch effective focal length parabolic mirror, forming a plasma inside the water film. Filters were placed to block the remaining laser beam as well as any white light simultaneously generated from the water film in addition to the $\mathrm{THz}$ radiation. A tungsten wire-grid polarizer was applied as the $\mathrm{THz}$ polarizer. Standard electro-optic sampling (EOS) [54] with a $3 \mathrm{~mm}$ thick $\langle 110\rangle$-cut ZnTe was used to detect the THz electric field. The flow velocity of the water is about $1.3 \mathrm{~m} / \mathrm{s}$, meaning that the water film flows about $1.3 \mathrm{~mm}$ between two laser pulses. This distance is much greater than the diameter of the focal spot of the laser beam, which indicates that each $\mathrm{THz}$ pulse will not be affected by previous interaction between the water and laser pulses.

\subsection{Terahertz Radiation from a Water Film}

By using the above experimental set-up, $\mathrm{THz}$ wave generation from a water film is achieved. The THz waveform is shown as curve B in Figure 2.5(a). To confirm that the $\mathrm{THz}$ radiation is mainly emitted from the water film rather than the air plasma, the water film is translated along the direction of laser propagation. The schema of relative positions between the water film and the plasma is shown on the left-hand side of Figure 2.5(a). The corresponding THz waveforms are plotted on the converse side of Figure 2.5(a). For curve A, the focal point of the laser is behind the film: the laser beam passes through the water and is focused to generate $\mathrm{THz}$ waves from an air plasma. For curve B, the focal point is near the center of the water film: a plasma is 


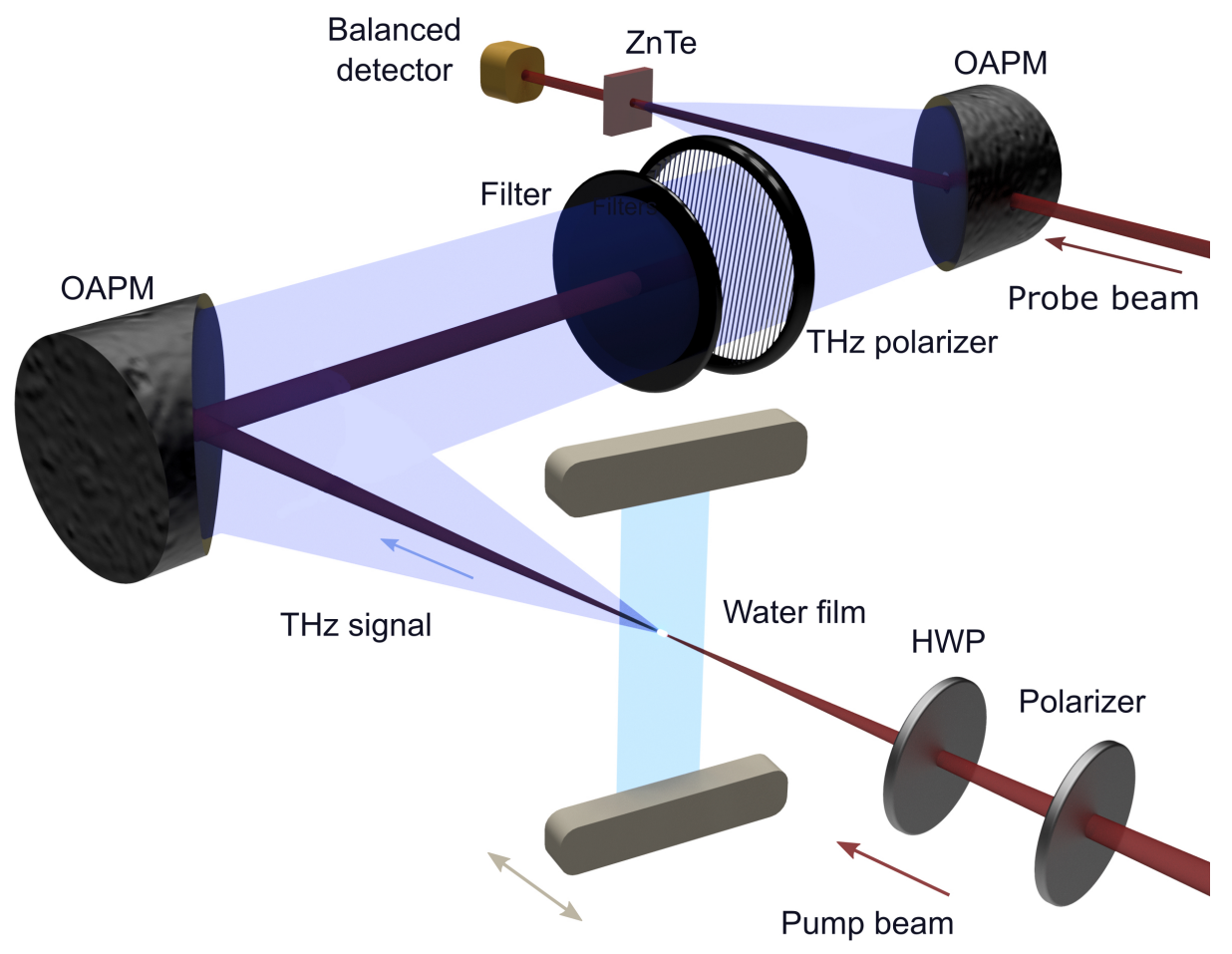

Figure 2.4: Experimental set-up for $\mathrm{THz}$ wave generation from a water film. Broadband $\mathrm{THz}$ wave is generated by tightly focusing the laser beam into a gravity-driven wire-guided free-flowing water film. The water film can be moved in the laser propagation direction by a mechanical translation stage. OAPM, off-axis parabolic mirror. HWP, half-wave plate. 
formed inside the water film, and the $\mathrm{THz}$ field emitted from liquid water is measured. For curve C, the laser beam is focused and forms an air plasma before the water film. Little $\mathrm{THz}$ radiation is detected due to the strong absorption of the water film. It is noticeable that the THz signals from air plasma will be clearly observed if the thickness of the water film is reduced to $100 \mu \mathrm{m}$ or less. Curve $\mathrm{D}$ is shown as a reference: no water film is present and only the $\mathrm{THz}$ wave generated from air plasma is detected.

By scanning the water film along the optical axis, THz radiation from different sources can be clearly differentiated. The timing distinctions of the waveforms in Figure 2.5(a) are indicative of different generation sources. A time delay is observed from the $\mathrm{THz}$ waveform from liquid water compared with other generations. Figure 2.5(b) shows the measurements of THz waveforms as the water film is tracked along the direction of laser propagation marking a relative position across $-60 \mu \mathrm{m}$ to $+60 \mu \mathrm{m}$. The measurement shows that the emitted $\mathrm{THz}$ waves are significantly sensitive to the relative position between the water film and the focus. The THz radiation can be detected only within a roughly $60 \mu \mathrm{m}$ scanning range of the water film. It should be mentioned that no $\mathrm{THz}$ radiation is detectable when only part of the plasma is located outside the range of the water film. The plasma located on the interface does not give a spurious $\mathrm{THz}$ signal. 
(a)

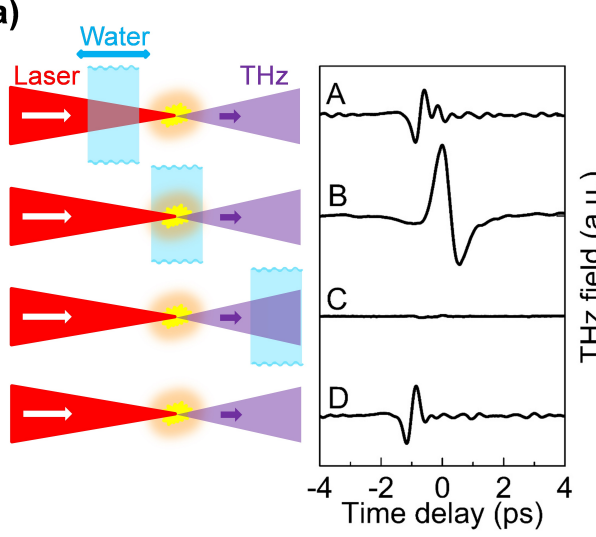

(b)

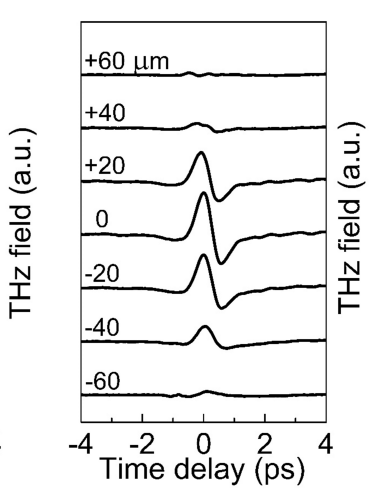

(c)

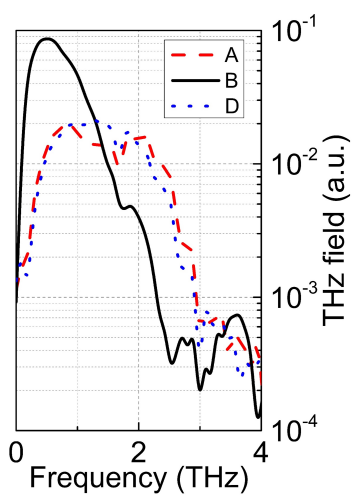

Figure 2.5: Measurements of the $\mathrm{THz}$ fields when the water film is translated along the direction of laser propagation. (a) $\mathrm{THz}$ waveforms are plotted from curve $\mathrm{A}$ to curve $\mathrm{C}$ when the water film is before, near, and after the focus, respectively; Curve $\mathrm{B}$ shows the $\mathrm{THz}$ waveform generated from liquid water; Curve D is the reference with no water film. Yellow spark and bluish pane represent the plasma and the water film respectively. $\mathrm{THz}$ emission angle shown in the figure is not meant to be indicative of actual $\mathrm{THz}$ emission pattern. (b) $\mathrm{THz}$ waveforms when the water film is moved near the focal point. The 0 position is set to the place with the strongest $\mathrm{THz}$ field. Relative positions are listed with the corresponding waveforms. The negative sign means the water film is located after the focal point. The positive sign indicates the opposite case. (c) Comparison between the $\mathrm{THz}$ field from water and that from air plasma in the frequency domain. The dashed, solid, and dotted spectra correspond to curve A, curve B, and curve $\mathrm{D}$ in (a), respectively. The laser pulse is temporally stretched to $550 \mathrm{fs}$ for these measurements. 


\subsection{Comparison between Terahertz Radiation from Water and Air}

Ambient air is one of the most extensively used $\mathrm{THz}$ sources. It is necessary to compare the $\mathrm{THz}$ radiation generated from water and air in the same experimental condition. Actually, curve B and curve D in Figure 2.5 offer a reasonable comparison. To make it clear, only these two signals are plotted in Figure 2.6. Figure 2.6 (a) depicts that the $\mathrm{THz}$ field from the water film is 1.8-times stronger than that from the air. The corresponding comparison in the frequency domain is shown in Figure 2.6 (b). Note that the feature around $1.7 \mathrm{THz}$ is caused by the water vapor absorption. The measured $\mathrm{THz}$ radiation from the water has more low-frequency components and less high-frequency components with a peak at $0.5 \mathrm{THz}$. In addition, the bandwidth is narrower than the signal from air plasma. Their difference in the frequency domain may result from the fact that liquid water absorbs more high-frequency components than low-frequency components in the THz frequency region (see Figure 1.9] [5]. It should be mentioned that the result in Figure 2.6 only shows the comparison in this experimental condition. The signal from liquid water will be rather stronger if other optimal conditions (the incident angle of the laser beam on the water film, the thickness of the water film, the detection angle, etc.) are satisfied, which will be discussed in later sections. 

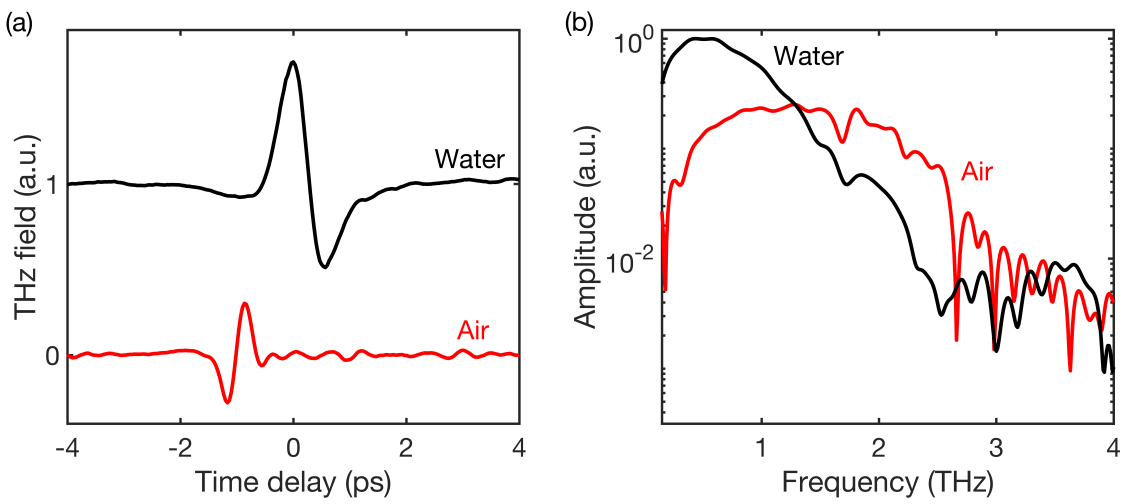

Figure 2.6: Comparison of $\mathrm{THz}$ waves generated from water and air in the (a) time domain and (b) frequency domain.

\subsection{Effect of Optical Polarization and Pulse En- ergy}

Polarization and pulse energy of the optical beam are two important parameters that may affect $\mathrm{THz}$ wave generation from liquid water.

The relationship between the $\mathrm{THz}$ radiation and the optical polarization is exposed by measuring the $\mathrm{THz}$ fields generated from the optical excitation beams with different polarizations. Since the water-air interface reflects more s-polarized $\mathrm{THz}$ waves according to the Fresnel equations, the p-polarized component of the $\mathrm{THz}$ field is studied here. A wire-grid $\mathrm{THz}$ polarizer was applied for the measurement. The polarization of the optical beam was controlled by the HWP in the optical path (see Figure 2.4). The corresponding result is shown in Figure 2.7. $0^{\circ}$ refers to the p-polarized optical beam and $90^{\circ}$ refers to the optical beam with s-polarization. It is shown that strong $\mathrm{THz}$ 


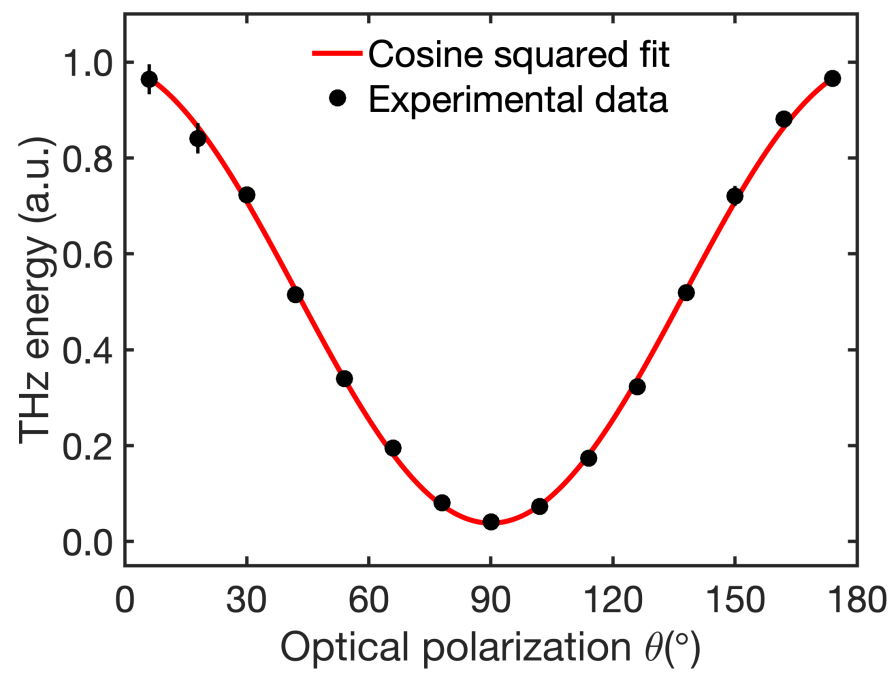

Figure 2.7: The energy of p-polarized $\mathrm{THz}$ field from liquid water with different linearly optical polarization. $0^{\circ}$ and $90^{\circ}$ refer to p-polarized and spolarized optical beam respectively.

radiation is achieved with a p-polarized optical beam, while an s-polarized optical beam offers a sparse contribution. One possible explanation is that the polarization of the $\mathrm{THz}$ field is dependent on the optical polarization, as the measured p-polarized component of the $\mathrm{THz}$ field has a cosine squared relationship with the angle of the optical polarization. Also, the different Fresnel loss of the optical beam caused by the air-water interface with different polarization will change the laser intensity inside the water film, thus, lead to the variation of $\mathrm{THz}$ radiation. A similar phenomenon was reported in [55] wherein a particle-in-cell model [56] was applied to simulate the observation. This result goes against the case of $\mathrm{THz}$ wave generation from one-color laser-induced air plasma. It is well known that the $\mathrm{THz}$ radiation 
from air plasma with one-color optical excitation does not depend upon the polarization of the optical beam [23, 24], which means that the THz energy keeps constant with various optical polarizations.

Furthermore, the linear energy dependence observed in Figure 2.8 is different from the quadratic relation in the case of $\mathrm{THz}$ wave generation from the one-color laser-induced air plasma [57]. Figure 2.8 also shows a laser excitation threshold at about $160 \mu \mathrm{J}$ for the detectable $\mathrm{THz}$ field from liquid water under this experimental condition. The $177 \mu \mathrm{m}$ thick water film will be broken if the energy of the excitation pulse is over $420 \mu \mathrm{J}$. This rupture may be caused by the occurrence of shock waves, plasma expansion, and water ejection when high-intensity laser pulses are focused into liquid water [58, 59, 60, 61, 62]. These effects weaken the stability of the water film as laser energy is increased.

\subsection{Effect of Water Film's Thickness}

The wire-guided free-flowing water film's thickness can be easily controlled by throttling the flow rate, which facilitates the study of how the water film's thickness impacts on the $\mathrm{THz}$ radiation. $\mathrm{THz}$ waveforms generated from water films with different thicknesses are plotted in Figure 2.9.

To make sure that the water film was not broken under the excitation of intense laser pulses, the thickness of the water film was set to be greater than $130 \mu \mathrm{m}$ in this measurement. Figure 2.9 shows that the peak of the $\mathrm{THz}$ 


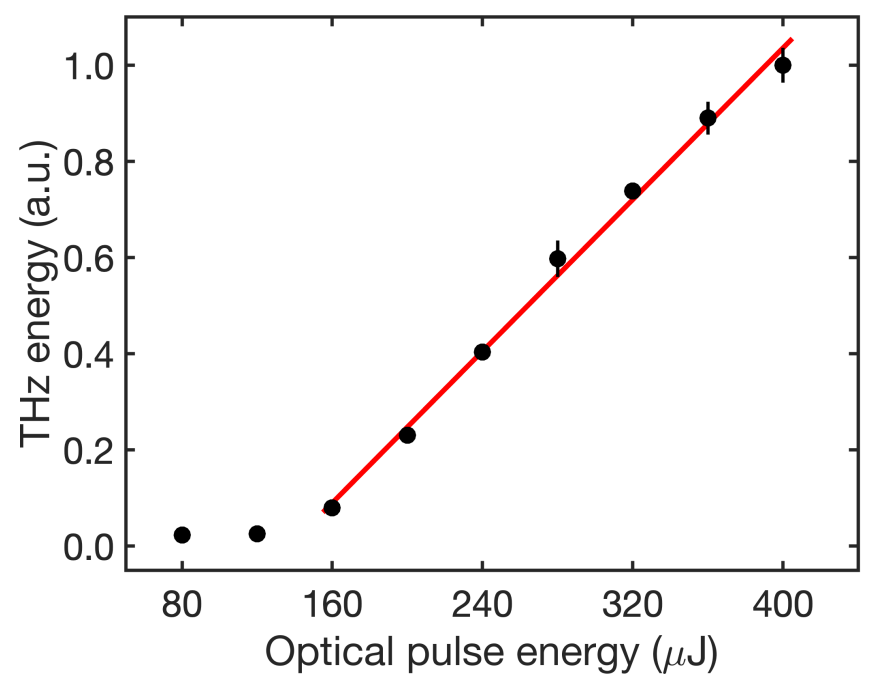

Figure 2.8: Normalized $\mathrm{THz}$ energy from liquid water as a function of incident optical pulse energy. The water film will be broken if the energy of the excitation pulse is over $420 \mu \mathrm{J}$.

waveform linearly shifts in time with various thicknesses. This time shift $\Delta t$ comes from the path difference of $\mathrm{THz}$ waves in water with difference thickness $\Delta d: \Delta t=n_{\mathrm{THz}} \Delta d / c$. The central frequency of the THz radiation is around $0.5 \mathrm{THz}$ in this case (see Figure 2.5(c) and Figure 2.6). From the linear fitting (red dash line), the refractive index of water at $0.5 \mathrm{THz}$ can be calculated as $n_{\mathrm{THz}}=2.29$ if increasing the thickness of the water film is assumed to go symmetrically with respect to the center of the film. Furthermore, the attenuation of the THz field's amplitude with the increased thickness is observed in Figure 2.9. The attenuation of amplitude $\Delta A$ results from the increasing absorption of a thicker water film as $\Delta A=\exp (-\alpha \Delta d)$. From the exponential fitting (red dash curve), the absorption coefficient of 
water at $0.5 \mathrm{THz}$ is calculated as $\alpha=146.2 \mathrm{~cm}^{-1}$. The calculated refractive index and absorption coefficient are very close to the values found in previous work: $n_{\mathrm{THz}}=2.27$ and $\alpha=150 \mathrm{~cm}^{-1}[5]$. It is noteworthy that only the thickness of the water film was changed, and no further optimizations were applied in this measurement. In Chapter 4, how the thickness impacts on the optimal conditions for $\mathrm{THz}$ radiation will be discussed.

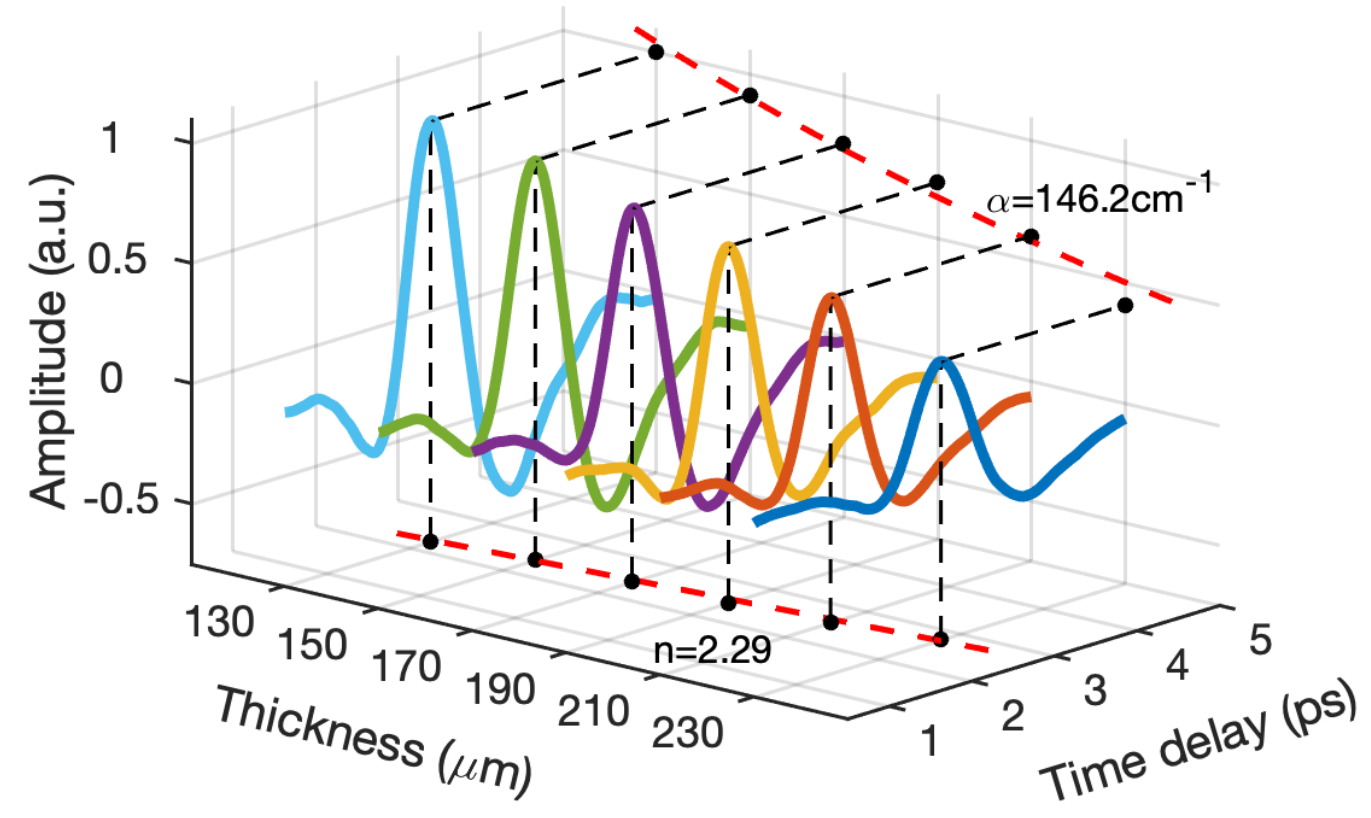

Figure 2.9: $\mathrm{THz}$ wave generation from water films with different thicknesses. The refractive index of water at $0.5 \mathrm{THz}$ is calculated to be 2.29 from the time shift of the $\mathrm{THz}$ field. The absorption coefficient of water at $0.5 \mathrm{THz}$ is calculated to be $146.2 \mathrm{~cm}^{-1}$ from the attenuation of the THz field's amplitude. 


\subsection{Forced-Flowing Thin Water Film}

$\mathrm{THz}$ wave generation from a gravity-driven free-flowing water film has already been demonstrated in Section 2.3. However, it is observed that the film will be broken if the laser intensity is too high, which may impede the development of intense liquid sources for $\mathrm{THz}$ waves. To solve this problem, a nozzle jet was utilized to produce a forced-flowing water film to bear intense laser pulse. A liquid jet with a pressure of 30 psi was used to create a $5 \mathrm{~mm}$ wide, 120 $\mu \mathrm{m}$ thick water film, as shown in Figure 2.10.

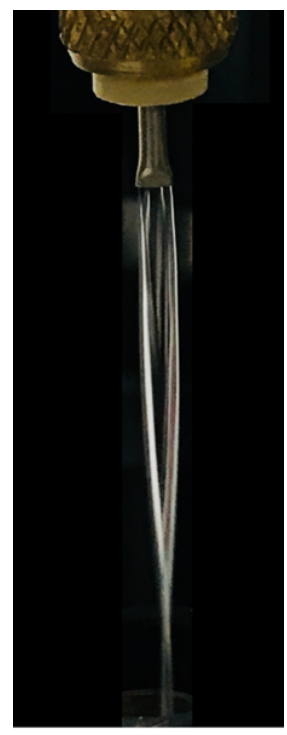

Figure 2.10: Photo of a $120 \mu \mathrm{m}$ thick water film formed by a water jet with a flat nozzle. The laser beam is focused into the center of the film where is flat and stable.

By using this forced-flowing water film, the laser pulse energy can be greatly increased without worrying about the occurrence of rupture, which 
assures that a plot of energy dependence with a large range can be seen. Similar to Figure 2.8, Figure 2.11 also shows that the $\mathrm{THz}$ energy linearly increases with the optical pulse energy (up to $2 \mathrm{~mJ}$ ). No saturation is observed within this range as well. This result is indicative of a way to realize intense $\mathrm{THz}$ radiation from water: using intense laser pulses for excitation.

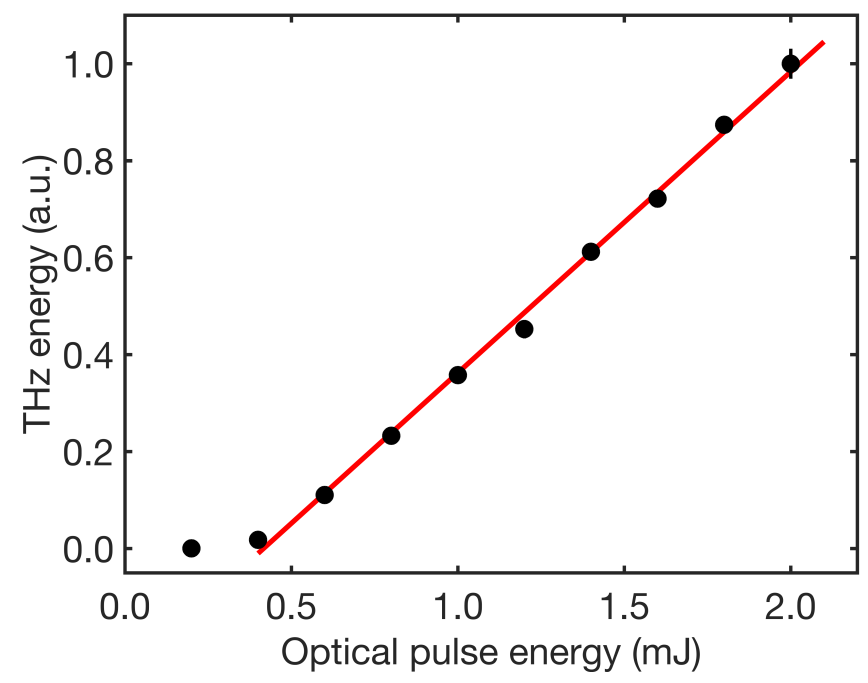

Figure 2.11: Normalized THz energy as a function of the optical pulse energy. The red line shows a linear fit.

\subsection{Mechanism of the Generation Process}

With these experimental observations, the physical mechanism of $\mathrm{THz}$ wave generation from water can be analyzed. Laser-induced plasma formation associated with a dipole radiation model is proposed to explain the generation process. 


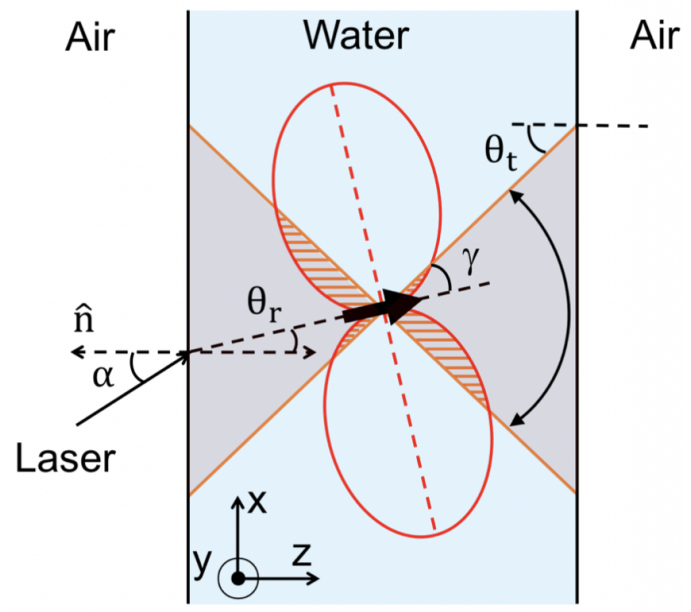

Figure 2.12: $2 \mathrm{D}$ cross-section of the $\mathrm{THz}$ wave generation process in a water film. Intense pulses ionize water at the focal point in the direction of the refracted laser beam. The angle of incidence on the air-water interface is $\alpha$. The black arrow shows the dipole orientation direction. Due to the total internal reflection at the water-air interface, $\mathrm{THz}$ emission at $0.5 \mathrm{THz}$ can be coupled out only when $-24.6^{\circ}<\theta_{\mathrm{t}}<+24.6^{\circ}$. 
Figure 2.12 is a cross-section diagram of the generation process in a water film. Intense laser pulses ionize water molecules through multi-photon ionization (MPI) [63, 64, 65, 66, 67], tunneling ionization (TI) 68, 69, and cascade ionization (avalanche ionization) [64, 65, 70, 71, 72, 73] in the focal volume. Plasmas (electrons and ions) are formed in the ionized area. The quasi-free electrons (will be called as free electrons in later sections for simplicity) in water [74] experience the ponderomotive force and move towards the areas of lower electron density due to the density-gradient distribution. Simultaneously, other ionized particles are relatively stationary due to their large masses. Since the electrons move slower than the envelope of the laser pulse, the density of the ionized carriers always keeps identical in the forward direction. As a result, electrons are accelerated backward and create a dipole oriented along the laser propagation direction [23, 24], which emits electromagnetic waves including $\mathrm{THz}$ frequencies. Reflection of the optical beam at the air-water interface and refraction of the $\mathrm{THz}$ beam at the waterair interface are considered. THz beams that experience multiple reflections between the interfaces are ignored due to the strong absorption of water.

\subsubsection{Laser-Induced Plasma Formation}

Electrons are produced through MPI/TI and cascade ionization at the focus. MPI/TI directly ionizes water molecules while cascade ionization desires the presence of free electrons for initiation. These free electrons come from the background electrons and MPI/TI. 

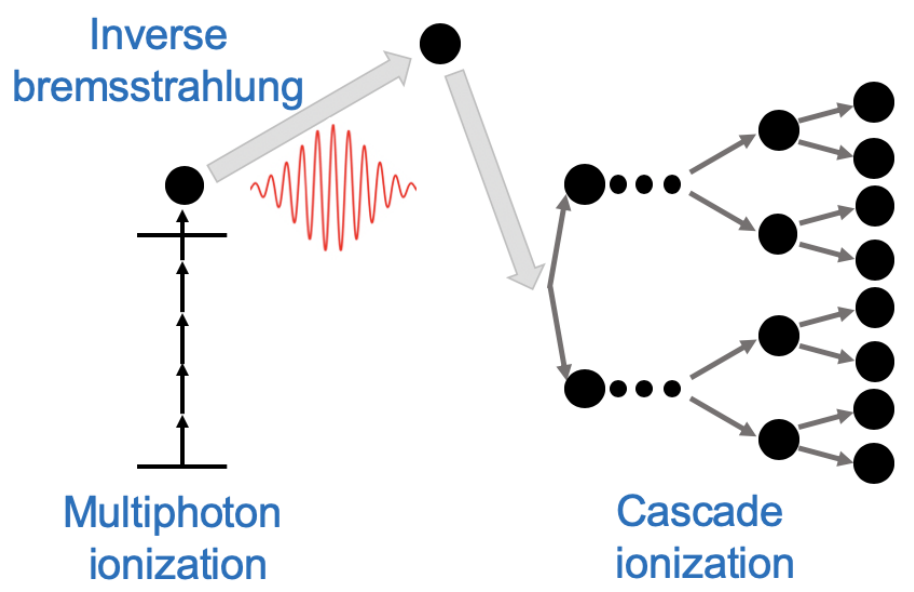

Figure 2.13: Visualization diagram of laser-induced plasma formation.

Free electrons absorb photons to gain energy from laser pulses through collision with surrounding atoms or molecules, which is known as inverse bremsstrahlung absorption. Water can be treated as an amorphous semiconductor with a bandgap of $E_{\mathrm{g}}=6.5 \mathrm{eV}$ [50, [51, 75, 76]. Once achieving the energy greater than the ionization energy $E_{\mathrm{g}}$, a free electron will be produced. This results in two free electrons of lower energy, which in tum absorb energy from the laser and ionize two more electrons, and so on. Thus, the process leads to a cascade of electrons. Figure 2.13 shows the process of plasma formation. It needs to be mentioned that TI occurs or dominates over MPI when the laser intensity is sufficiently high to decrease the potential barrier allowing electrons to escape. The Keldysh parameter $\gamma$ is widely used to 
indicate which process is dominant.

$$
\gamma=\sqrt{E_{\mathrm{g}} / U_{\mathrm{p}}}
$$

where $U_{\mathrm{p}}$ is the laser ponderomotive potential energy. $\gamma>1$ implies MPI while $\gamma<1$ indicates TI.

Assuming the laser pulse propagates in the $z$-direction, the electric field envelope $E(z, r, t)$ follows the equation [77]:

$$
\frac{\partial E}{\partial z}=\frac{i}{2 n k_{0}} \nabla_{T}^{2} E+i k_{0} n_{2}|E|^{2} E-\frac{i \beta_{2}}{2} \frac{\partial^{2} E}{\partial t^{2}}-\frac{\sigma}{2}(1+i \omega \tau) \rho E-\frac{\beta^{(K)}}{2}|E|^{2 K-2} E .
$$

The terms on the right-hand side represent transverse beam diffraction, nonlinear self-focusing, group velocity dispersion (GVD), plasma absorption and defocusing, and multiphoton absorption, respectively. Here, $n$ is the refractive index, $k_{0}$ is the wave vector, $n_{2}$ is the nonlinear coefficient, $\beta_{2}=\partial^{2} k / \partial \omega^{2}$ represents GVD, $\sigma=\left(k e^{2} \tau / m \omega \epsilon_{0}\right) /\left(1+\omega^{2} \tau^{2}\right)$ is the cross-section for inverse bremsstrahlung absorption [78], $\omega$ is the optical frequency, $\tau$ is the electron collision time, and $\beta^{(K)}$ is the nonlinear coefficient for $K$-photon absorption. The last term need be modified to $\frac{w\left(\rho_{\text {water }}-\rho\right) E_{\mathrm{g}}}{2 I} E$ when TI dominates [79]. $w$ is the ionization rate, $\rho_{\text {water }}$ is the water molecular density, and $I$ is the laser intensity. The electron density $\rho(z, r, t)$ satisfies the rate equation [74]:

$$
\frac{\partial \rho}{\partial t}=\left(\frac{\partial \rho}{\partial t}\right)_{\mathrm{mp}}+\eta_{\mathrm{cas}} \rho-\eta_{\mathrm{diff}} \rho-\eta_{\mathrm{rec}} \rho^{2}
$$


The first two terms on the right-hand side correspond to the generation of electrons from MPI and cascade ionization. The first term becomes $w\left(\rho_{\text {water }}-\right.$ $\rho$ ) in the case of TI. The other two terms in Equation 2.3 describe the loss of electrons from diffusion and recombination. The laser pulse is considered to have a Gaussian profile with its intensity as follows:

$$
I(t)=0.94 \frac{\varepsilon_{\mathrm{p}}}{\tau_{\mathrm{p}}} \exp \left[-4 \ln 2\left(\frac{t}{\tau_{\mathrm{p}}}\right)^{2}\right] /\left(0.5 \pi w_{0}^{2}\right)
$$

where $\varepsilon_{\mathrm{p}}$ is the laser pulse energy, $\tau_{\mathrm{p}}$ is the laser pulse duration, and $w_{0}$ is the beam waist at the focus.

In condensed media, Keldysh [68] derived an approximate expression for the multiphoton ionization rate as [50, 74]:

$$
\left(\frac{\partial \rho}{\partial t}\right)_{\mathrm{mp}} \approx \frac{2 \omega}{9 \pi}\left(\frac{m \omega^{2}}{2 \hbar \omega}\right)^{1.5}\left(\frac{e^{2}}{8 m E_{\mathrm{g}} \omega^{2} c \epsilon_{0} n} I\right)^{K}+\exp (2 K) \Phi\left(\sqrt{2 K-\frac{2 E_{\mathrm{g}}}{\hbar \omega}}\right)
$$

with $\Phi(x)=\exp \left(-x^{2}\right) \int_{0}^{x} y^{2} \mathrm{~d} y$.

In the case of $\mathrm{TI}$, the ionization rate can be calculated by AmmosovDelone-Krainov (ADK) model [69]:

$$
w(t)=4 \omega_{a} \frac{E_{a}}{|E|} \exp \left(-\frac{2 E_{a}}{3|E|}\right)
$$

The cascade ionization rate is given by [50, 74]:

$$
\eta_{\text {cas }}=\frac{\sigma}{n^{2} E_{\mathrm{g}}} I-\frac{m \omega^{2} \tau}{M\left(1+\omega^{2} \tau^{2}\right)}
$$


where $M$ is the mass of a water molecule. The first term on the right-hand side results from the inverse bremsstrahlung absorption [74, 77]. The second term relates to the energy transfer from the electrons to heavy molecules [74, 80].

\subsubsection{Dipole Radiation Model}

The ionized electrons are accelerated to form a dipole in the direction of the refracted laser beam that emits $\mathrm{THz}$ radiation as shown in Figure 2.12 . To confirm the applicability of the dipole radiation model, the following experiments were conducted. The laser pulses with horizontal polarization, $800 \mathrm{~nm}$ central wavelength, and $1 \mathrm{kHz}$ repetition rate were delivered from a Ti: sapphire amplified laser. It was focused into a $120 \mu \mathrm{m}$ thick water film by a 2 -inch effective focal length lens $(\mathrm{F} / 4)$ ionizing water molecules at the focus. A $2 \mathrm{~mm}$ thick $\langle 110\rangle$-cut ZnTe crystal configured for EOS [54] and a commercially available Golay cell were both used for the detection of $\mathrm{THz}$ waves. An illustration of the incident angle $\alpha$ for the laser beam and the angle of detection $\beta$ for the detector is shown in Figure 2.14. These two angles are both defined with respect to the laser propagation direction (z-axis) and can be changed by rotating the film and detector, respectively. $\hat{n}$ is the surface normal of the water film. The sign of the angle is negative/positive when it is measured clockwise/counter-clockwise from the $z$-axis. The optical pulse duration is tuned to be $300 \mathrm{fs}$ for maximizing the THz signal with the 120 $\mu \mathrm{m}$ thick water film. 


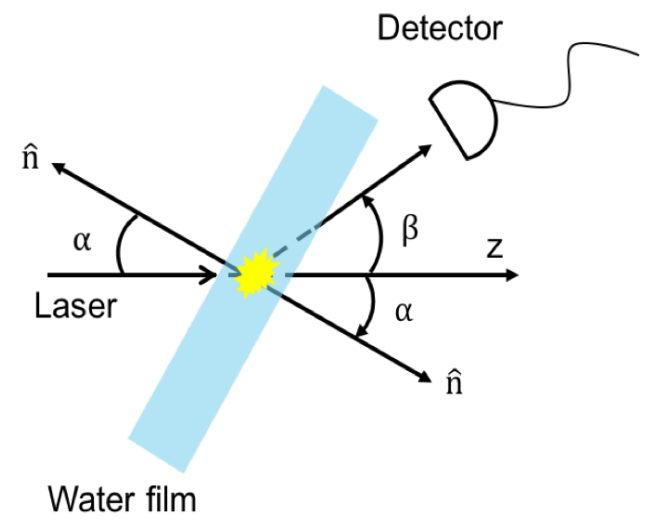

Figure 2.14: Illustration of incident angle $\alpha$ and angle of detection $\beta$. All angles are defined with respect to the $z$-axis.

Figure 2.15 plots the THz waveforms generated from the water film with two opposite angles of incidence $\left(\alpha= \pm 65^{\circ}\right)$ and detected in the laser propagation direction. The corresponding spectra are shown in the inset. The central wavelength and full width at the half maximum (FWHM) are both about $0.5 \mathrm{THz}$. Therefore, the parameters (index of refraction and absorption coefficient) at $0.5 \mathrm{THz}[5]$ are used in the following calculation and simulation. As show in Figure 2.15, the waveform keeps its amplitude but completely flips over when the angle of incidence changes its sign. This observation applies to all opposite angles of incidence. It can be inferred that the dipole orientation is along the propagation direction of the refracted laser beam, illustrated as a black arrow in Figure 2.15. The projected signal from the dipole reverses in direction when the angle of incidence changes the sign, which accounts for the flipped waveforms. 


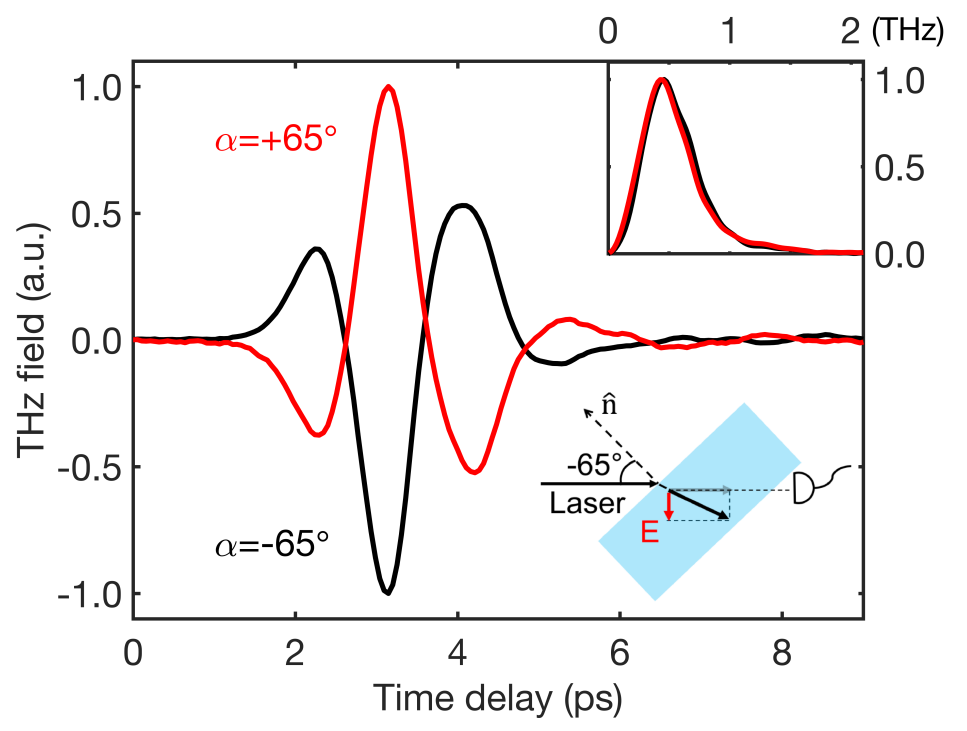

Figure 2.15: $\mathrm{THz}$ waveforms generated from a water film with opposite angles of incidence $\left(\alpha= \pm 65^{\circ}\right)$. The corresponding spectra and dipole approximation illustration are shown in the insets.

\subsection{Radiation Pattern}

In the last section, a dipole model was proposed to explain the mechanism of $\mathrm{THz}$ wave generation from liquid water. Similar to the case in air, a spatial net charge distribution created by the ponderomotive force acts like a Hertzian dipole and radiates $\mathrm{THz}$ waves [23, 24]. However, the existence of the interfaces makes the scenario more complicated. Specifically, the refraction of the laser beam induced by the first interface (air-water) changes the orientation of the dipole while the total internal reflection and refraction of the $\mathrm{THz}$ beam caused by the second interface (water-air) significantly affect the radiation angle of $\mathrm{THz}$ waves. The model also predicts that backward 
$\mathrm{THz}$ radiation should be present even though only the forward radiation has been paid attention in the previous study. Thus, simulations and measurements of the $\mathrm{THz}$ radiation pattern can be very useful to further verify the dipole radiation model.

At the air-water interface, refractive angle $\theta_{\mathrm{r}}(\alpha)$ and transmittance $T_{1}(\alpha)$ for a given $\alpha$ is obtained according to Snell's law and the Fresnel equations, which are determined by the refractive indices of the $800 \mathrm{~nm}$ optical beam in air and water. The maximum $\theta_{\mathrm{r}}(\alpha)$ is $48.8^{\circ}$ when $\alpha=90^{\circ}$. THz waves radiated by the dipole propagating in the water film are attenuated due to the absorption of water. If the thickness of the water film is $d$, the absorption in the different direction $\theta_{\mathrm{t}}(\beta)$ from the source can be described as $\exp [-$ $\left.\alpha_{\mathrm{THz}} d / 2 \cos \theta_{\mathrm{t}}(\beta)\right]$, where $\alpha_{\mathrm{THz}}$ is the power absorption coefficient of water. Multiple reflections of $\mathrm{THz}$ waves are neglected in the calculation due to the strong absorption of water. Additionally, the dipole radiation energy is proportional to $\sin ^{2}(\gamma)$, where $\gamma(\alpha, \beta)=\theta_{\mathrm{t}}(\beta)-\theta_{\mathrm{r}}(\alpha)$ is the angle measured with respect to the dipole direction. Finally, the $\mathrm{THz}$ waves are detected after passing through the water-air interface with a transmittance $T_{2}(\beta)$. To sum up, the angular dependence of $\mathrm{THz}$ energy on $\alpha$ and $\beta$ is described as:

$$
I_{\mathrm{THz}}(\alpha, \beta) \propto T_{1}(\alpha) T_{2}(\beta) \sin ^{2}(\gamma)(\alpha, \beta) \exp \left(-\frac{\alpha d}{2 \cos \theta_{t}(\beta)}\right) .
$$

Figure 2.16 plots the simulation result of the normalized $\mathrm{THz}$ energy $I_{\mathrm{THz}}(\alpha, \beta)$. A micro-plasma is created in the water film with the tightly 


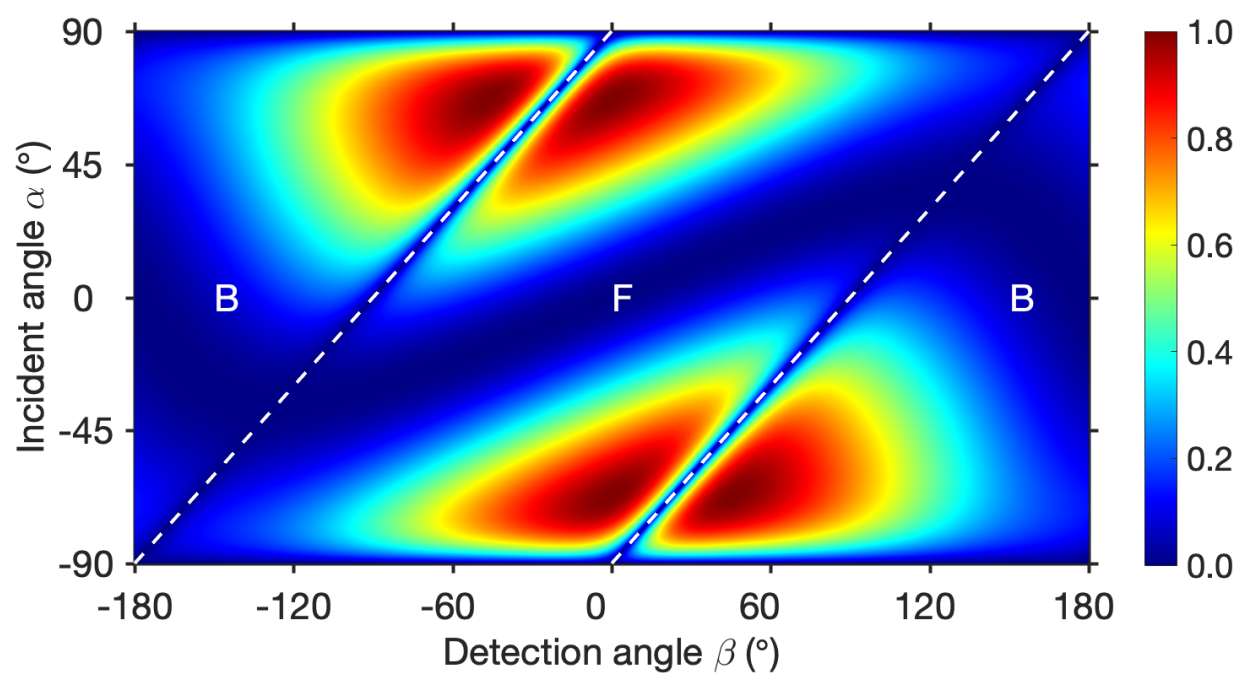

Figure 2.16: Simulation result of normalized THz energy $I_{\mathrm{THz}}(\alpha, \beta)$ using the dipole radiation model. The dashed lines indicate the cases of $|\alpha-\beta|=90^{\circ}$, which means the detector is located in the plane of the water film. These dash lines separate the plots into three parts, labeled as "B", "F", and "B". "B" and " $\mathrm{F}$ " indicate backward and forward propagating $\mathrm{THz}$ signal, respectively. 
focused geometry [57]. This plasma can be considered as a point source emitting $\mathrm{THz}$ waves in all directions. Thus, besides the forward (F) propagating signal, the signal propagating in the backward (B) direction is also expected. These two parts are separated by the dashed line in the plot and labeled separately. Due to the symmetric geometry of the model, the energy distribution pattern for forward and backward propagating $\mathrm{THz}$ signals are the same when the plasma is located at the center of the water film. The dashed lines also indicate the case of $|\alpha-\beta|=90^{\circ}$, which means that the detector is put in the plane of the water film. When $|\alpha-\beta|>90^{\circ}$, the $\mathrm{THz}$ waves propagate in the backward direction.

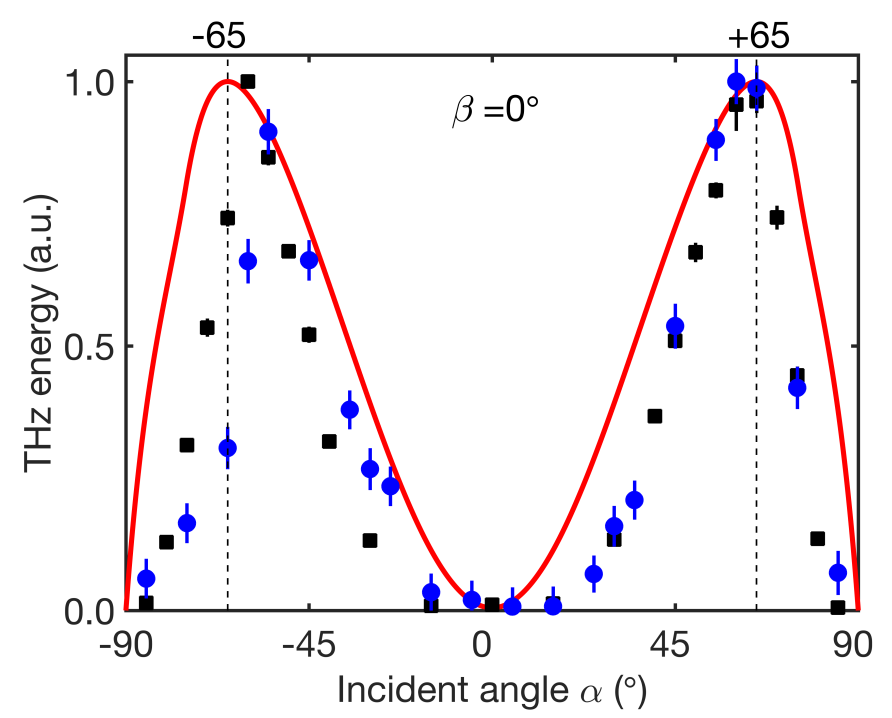

Figure 2.17: THz energy versus the angle of incidence $\alpha$ with $\beta=0^{\circ}$. The black squares are the data measured by EOS and the blue dots are measured by a Golay cell. Only forward propagating signals can be detected for $\beta=$ $0^{\circ}$. 
To verify the simulation result, the $\mathrm{THz}$ signal versus $\alpha$ was measured when $\beta$ was fixed at $0^{\circ}$ or $55^{\circ}$, both EOS and a Golay cell were used for the detection in the measurement. The corresponding results are plotted in Figure 2.17. The red solid line shows the simulation result. The EOS result (black squares) is obtained from the temporal integration of the whole $\mathrm{THz}$ waveform. The result from the Golay cell is plotted as the blue dots. As shown in the plot, the optimal incident angle of the laser beam is $65^{\circ}$, which results from the dipole's orientation. The coincidence of the experimental data and the simulation exhibits the validity of the dipole radiation model. Note that only the forward $\mathrm{THz}$ waves can be measured when $\beta=0^{\circ}$. In addition, the calculation with $\alpha=65^{\circ}$ indicates that $80 \%$ of the THz energy dissipates due to the total internal reflection at the water-air interface and the strong absorption of water.

Even though EOS generally offers a better signal-to-noise ratio, its optical alignment for the detection of radiation pattern is complicated. By contrast, a Golay cell is capable of measuring radiation pattern including both forward and backward directions easily. Thus, the Golay cell is used for the case of $\beta$ $=55^{\circ}$ (Figure 2.18). In this case, the signal comes from the backward radiation $\mathrm{THz}$ waves is observed when $90^{\circ}<\alpha<-35^{\circ}$. The detectable backward propagating $\mathrm{THz}$ signal supports the dipole radiation model. Compared to the simulation result (solid line), stronger signals are measured in the forward direction. This may be a consequence of plasma deviation from the center of the film. 


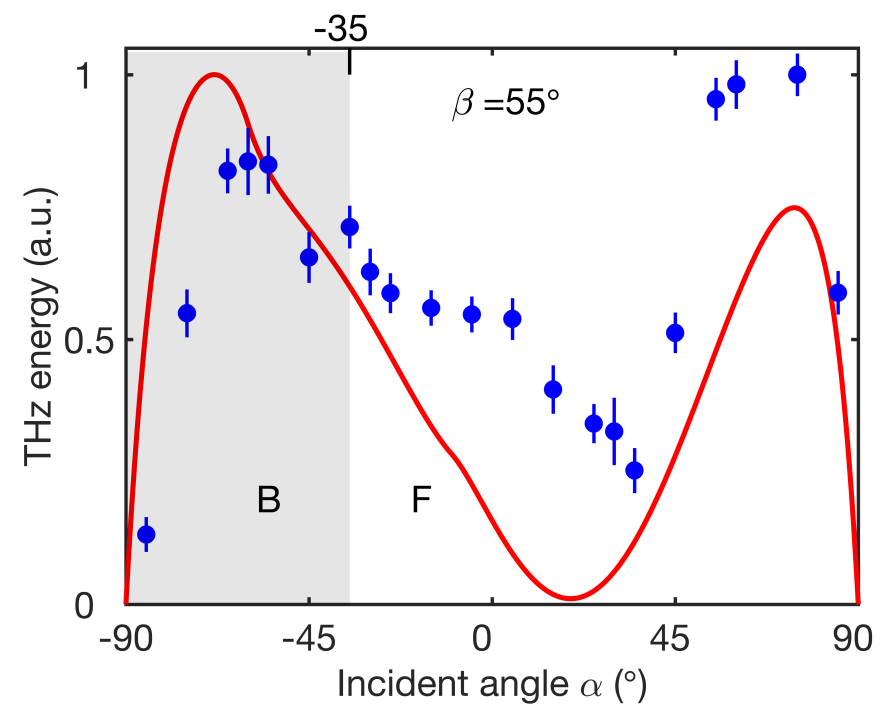

Figure 2.18: $\mathrm{THz}$ energy versus the angle of incidence $\alpha$ with $\beta=55^{\circ}$. The blue dots are measured by a Golay cell. Backward propagating signals are detected for $-90^{\circ}<\alpha<-35^{\circ}$.

\subsection{Effect of Optical Pulse Duration}

To study the dependence of the $\mathrm{THz}$ radiation on the optical pulse duration, the laser pulse was stretched in time to achieve different pulse duration. Figure 2.19 shows the normalized $\mathrm{THz}$ energy from water and air plasma versus the optical pulse duration. The $\mathrm{THz}$ energy from water or air plasma is normalized to the corresponding maximum respectively.

The optical pulse duration is at its minimum of 58 fs when no chirp is applied. The left-hand side of Figure 2.19 shows the case of negative chirps, where the low-frequency component of the pulse lags the high-frequency component. Positive chirps indicate the opposite and the corresponding measure- 


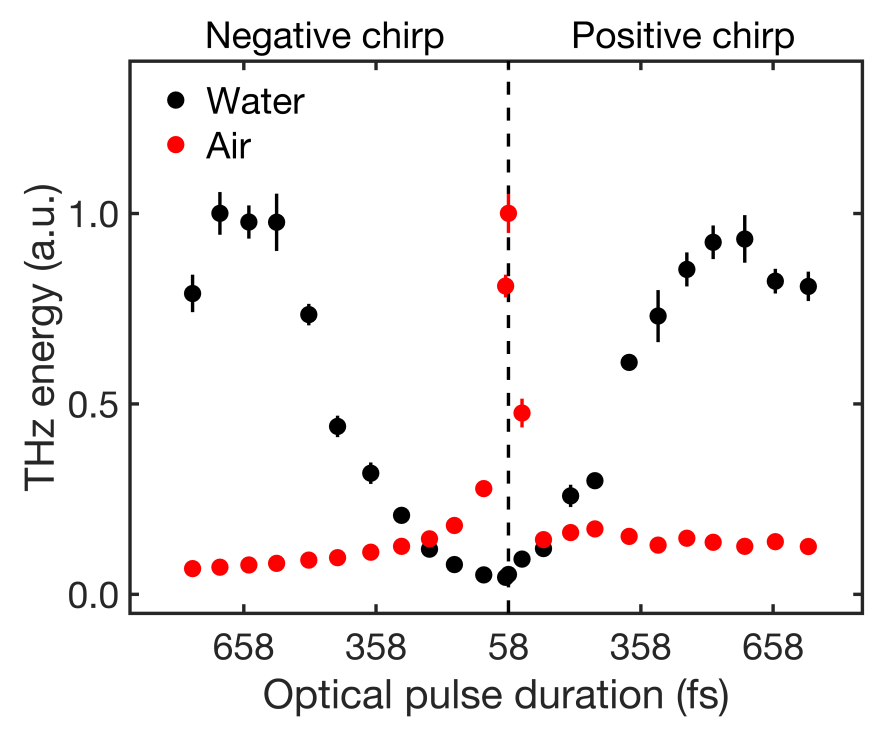

Figure 2.19: Normalized THz energy from liquid water and air plasma with different pulse duration of the laser beam. Black squares represent the $\mathrm{THz}$ energy from liquid water and red dots represent the case of air plasma. The optical pulse duration is at its minimum of 58 fs when no frequency chirp is applied. On the left-hand side of the figure, negative chirps are applied to increase the optical pulse duration while the case of positive chirps is shown on the right-hand side of the figure. The energy of the laser pulse is $0.4 \mathrm{~mJ}$ for these measurements. 
ments are shown on the right-hand side of Figure 2.19 .

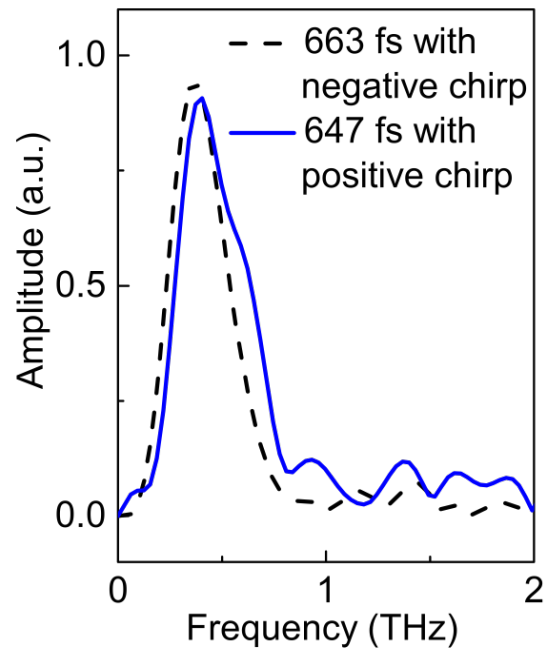

Figure 2.20: Spectra of $\mathrm{THz}$ radiation generated from the water film with a negative chirped $663 \mathrm{fs}$ pulse and that with a positive chirped $647 \mathrm{fs}$ pulse.

Unlike the $\mathrm{THz}$ radiation from air plasma, where the signal is maximized at a minimum pulse duration with no additional chirp, liquid water generates a maximum field at a longer pulse duration. Furthermore, by comparing the left part and the right part of Figure 2.19, it is shown that the frequency chirp of the optical beam is not a dominant factor compared with the contribution from the pulse duration. This can also be supported by Figure 2.20, which plots the similar spectra of $\mathrm{THz}$ radiation by using the same optical pulse duration but opposite chirps. For simplicity, only positive chirps will be applied in future investigation. 
The above observations may result from the dependence of plasma formation in water upon the optical pulse duration. To confirm the assumption, further experiments and simulations are indispensable. More in-depth discussions will be shown in Chapter 4.

\subsection{Summary}

In this chapter, the use of a liquid water film as a broadband $\mathrm{THz}$ wave source is demonstrated. It is also observed that liquid water generates a stronger $\mathrm{THz}$ electric field than air does under the same experimental condition. In addition, the $\mathrm{THz}$ field exhibits the sensitivity to the relative position between the laser focal point and the water film. The mechanism of the generation process is understood from the process of laser-induced plasma formation associated with a dipole radiation model.

The ponderomotive force-induced dipole model is supported by the observation that the $\mathrm{THz}$ waveforms completely flip over when the angle of incidence changes the sign. For further verification, the $\mathrm{THz}$ radiation pattern from the water film is experimentally measured. Both forward radiation and backward radiation are detected. The measurements match with the simulations that predicted by the dipole radiation model.

One important observation in water is that a longer optical pulse duration is required to obtain the strongest $\mathrm{THz}$ signal, which differs a lot from the case of air plasma. Further investigation into the preference for the long 
pulse duration will be discussed in Chapter 4 


\section{Chapter 3}

\section{Terahertz Radiation from Liquid Water under Two-Color Excitation Scheme}

Broadband $\mathrm{THz}$ wave generation from liquid water was demonstrated in the last chapter. In the following four chapters, various methods for boosting the $\mathrm{THz}$ radiation from liquid water will be successively discussed. The approach of using the two-color excitation scheme is the main topic in this chapter. First of all, a historical review of $\mathrm{THz}$ wave generation from gases under the two-color excitation scheme is briefly presented. Then it is experimentally demonstrated that liquid water excited by intense two-color laser pulses also emits $\mathrm{THz}$ waves. Compared with the one-color excitation, one-order of magnitude enhanced $\mathrm{THz}$ electric field is observed by using asymmetric optical excitation with the same total excitation pulse energy and focusing geometry. Modulation of the $\mathrm{THz}$ field is achieved via the coherent control approach. The modulated and unmodulated $\mathrm{THz}$ energy have respectively quadratic and linear dependence on the optical pulse energy. 


\subsection{Glory of Two-Color Excitation Scheme in Gases}

$\mathrm{THz}$ wave generation through the tunneling ionization process in gases induced by two-color (fundamental frequency $\omega$ and its second-harmonic $2 \omega$ ) femtosecond laser pulses [25, 81] is a significant milestone in the THz community due to its impressive intensity, remarkably broad bandwidth, and applications in nonlinear interactions and THz spectroscopy [82, 83, 84]. There is no exaggeration to say that THz wave generation under the two-color excitation scheme has been always one of the most popular topics since it was reported by Cook and Hochstrasser in 2000 [25]. A typical schematic diagram is shown in Figure 3.1. An intense femtosecond laser pulse $\omega$ and its second harmonic $2 \omega$ are focused into the air to generate air plasma for the emission of THz waves. Commonly, the $2 \omega$ laser pulse is generated by applying a type-I $\beta$-barium borate (BBO) crystal to the $\omega$ laser pulse. The output THz radiation is controlled by the phase delay between $\omega$ pulse and $2 \omega$ pulse. Compared with using only $\omega$ pulses for the excitation, the mixing of $\omega$ and $2 \omega$

pulses has shown to provide enhancement of $\mathrm{THz}$ wave generation efficiency by several orders of magnitude [25].

The generation mechanism of the two-color excitation scheme was phenomenologically assigned to a four-wave-mixing model as:

$$
E_{\mathrm{THz}}(t) \propto \chi^{(3)} E_{2 \omega}(t) E_{\omega}^{*}(t) E_{\omega}^{*}(t) \cos \phi,
$$




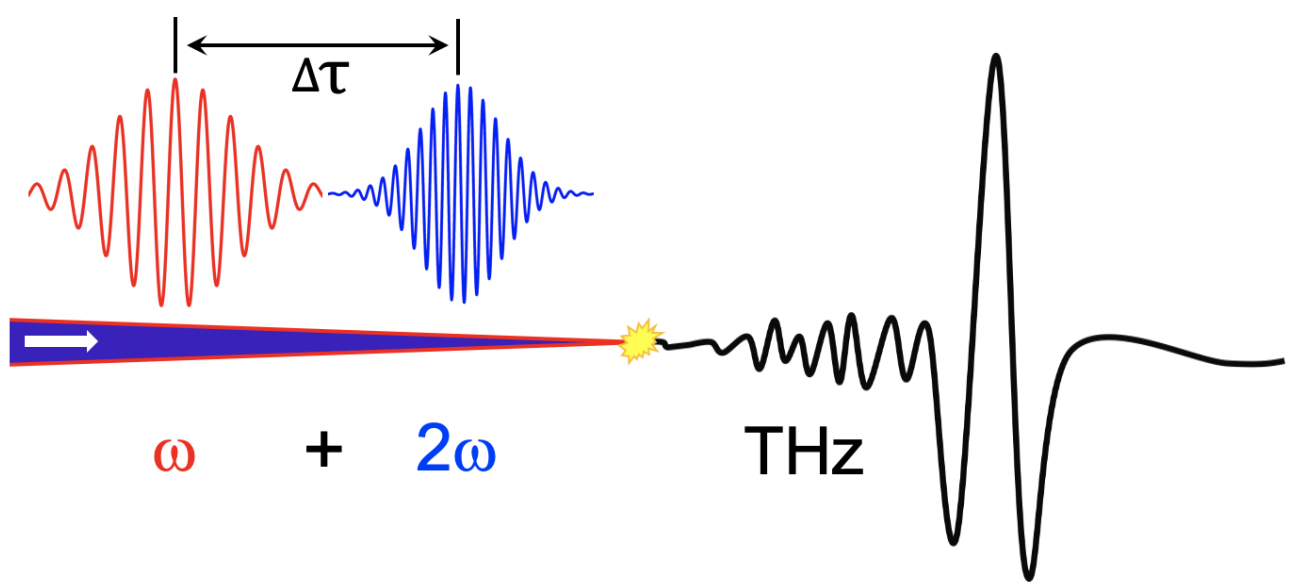

Figure 3.1: Schematic diagram of $\mathrm{THz}$ wave generation from a two-color laser pulses induced air plasma. An intense femtosecond laser beam $\omega$ and its second harmonic $2 \omega$ are focused to generate plasma in the air. In the most common way, a $\beta$-BBO crystal is applied for the generation of $2 \omega$ pulse. The output $\mathrm{THz}$ waves are determined by the phase delay between $\omega$ pulse and $2 \omega$ pulse. 
where $E$ is the field amplitude of the frequency components specified by the subscript, $\chi^{(3)}$ is the effective component of the third-order nonlinear susceptibility tensor of the gas, and $\phi$ is the relative phase between $\omega$ pulse and $2 \omega$ pulse. The relationship in Equation 3.1 was experimentally demonstrated from an energy dependence measurement by Xie et al. in 2006 [6]. Figure 3.2 shows the result: the $\mathrm{THz}$ amplitude is linearly increased with the intensity of $\omega$ pulse and is proportional to the square root of the intensity of $2 \omega$ pulse. Even though the four-wave-mixing model successfully offers an intuitive and straightforward description, it fails to explain some observations in experiments. For example, the occurrence of a clear threshold in Figure 3.2 cannot be predicted by the model. Instead, the threshold should be associated with the photoionization process happened in the air. Additionally, the four-wave-mixing model is not indicative of the enhancement of THz radiation that caused by an applied asymmetric laser field.

In 2007, Kim et al. proposed a transient photocurrent model [27] that could solve the problems mentioned above. In this model, a net photocurrent produced by asymmetric optical fields through the photoionization radiates $\mathrm{THz}$ waves. Compared with $\mathrm{THz}$ wave generation from gas plasmas induced by one-color laser pulses, the asymmetric electron motion introduced by the two-color laser fields leads to a net dipole moment and hence, much stronger THz emission.

In 2009, Karpowicz and Zhang developed a full quantum model [30] to depict a complete physical picture of the generation process by numerically 

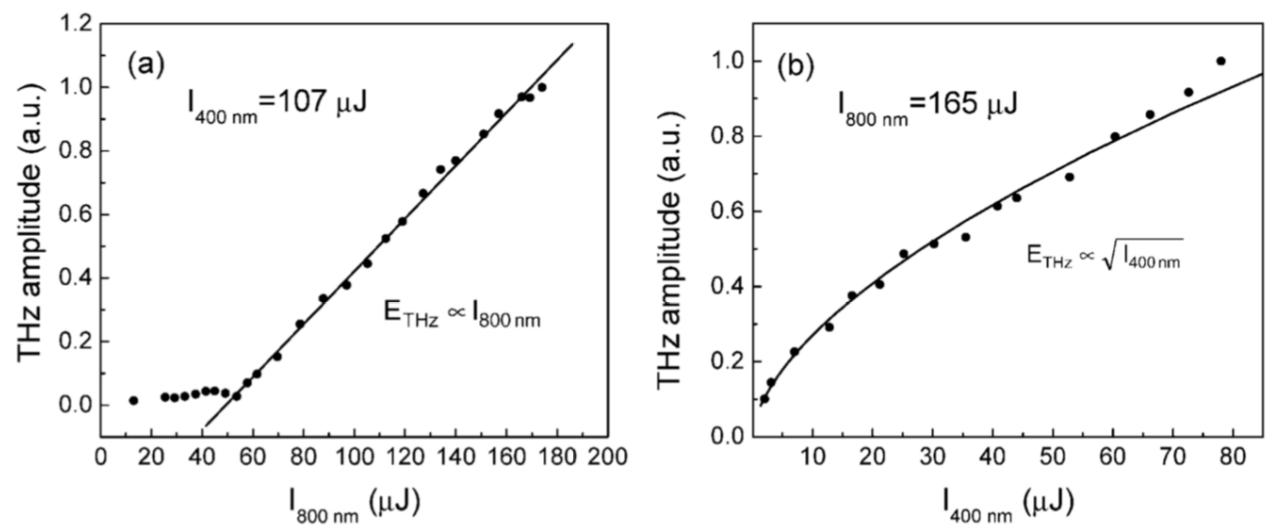

Figure 3.2: Dependence of $\mathrm{THz}$ amplitude on the power of the (a) $\omega$ and (b) $2 \omega$ beams in $\mathrm{THz}$ wave generation by two-color laser beams. (Reprinted with permission from [6], copyright 2006 American Physical Society)

solving the time-dependent Schrödinger equation. The model accurately describes the formation and acceleration of the relevant wave packets.

Compared with other $\mathrm{THz}$ wave generation techniques, the two-color excitation scheme in gases provides the $\mathrm{THz}$ radiation with intense electrical fields $(>8 \mathrm{MV} / \mathrm{cm})$ as well as broad spectral information $(>100 \mathrm{THz})$ [85, 86, 87].

In addition, the two-color laser pulses enable the coherent control of $\mathrm{THz}$ waves (see Figure 3.3 [7]. A femtosecond $\omega$ pulse generates a $2 \omega$ pulse while passing through a $\beta$-BBO crystal. The perpendicular polarized $\omega$ and $2 \omega$ pulses pass through an $\alpha$-BBO with its slow axis aligned with the polarization of the $\omega$ beam and the fast axis aligned with the polarization of the $2 \omega$ beam. Thus, the $2 \omega$ pulse leads the $\omega$ pulse after passing through the $\alpha$-BBO crystal [7], as shown in Figure 3.3(a). A fused silica wedge pair is used to finely 
control the phase delay between the $\omega$ and $2 \omega$ pulses. Finally, a tunable dual-band wave plate is used to control the polarization of the $\omega$ beam and the $2 \omega$ beam.

In Figure 3.3(b), modulation of $\mathrm{THz}$ waves generated from air plasmas has been achieved by changing the relative phase $\phi$ between $\omega$ and $2 \omega$ pulses via the phase compensator. The dependence of $\mathrm{THz}$ yield upon the relative phase $\phi$ in the case of gas plasmas was also investigated in $[7,27,28,29,30,88$. Moreover, the two-color excitation scheme was applied to thin metal films to achieve coherent control [89].

It is worth underlining that Kumar et al. also reported $\mathrm{THz}$ wave generation from liquids by focusing femtosecond laser pulses into a cuvette filled with target liquids [90]. Their observations also indicate that the laserinduced plasma formation in liquid materials plays a critical role in the $\mathrm{THz}$ wave generation process. The electrons ionized from water molecules are regarded as quasi-free electrons [74], and therefore the THz wave generation process in water resembles the process in air. Thus, the two-color excitation scheme should also work in liquid water. Stronger THz waves and corresponding modulation are expected by using the asymmetric excitation scheme in water as well. Manipulation of strong THz emission would have widespread applications in different research fields, such as THz nonlinear optics [91, 92] and electron acceleration [93, 94]. In the following sections, the $\mathrm{THz}$ wave generation from a thin water film under two-color laser excitation will be discussed. 

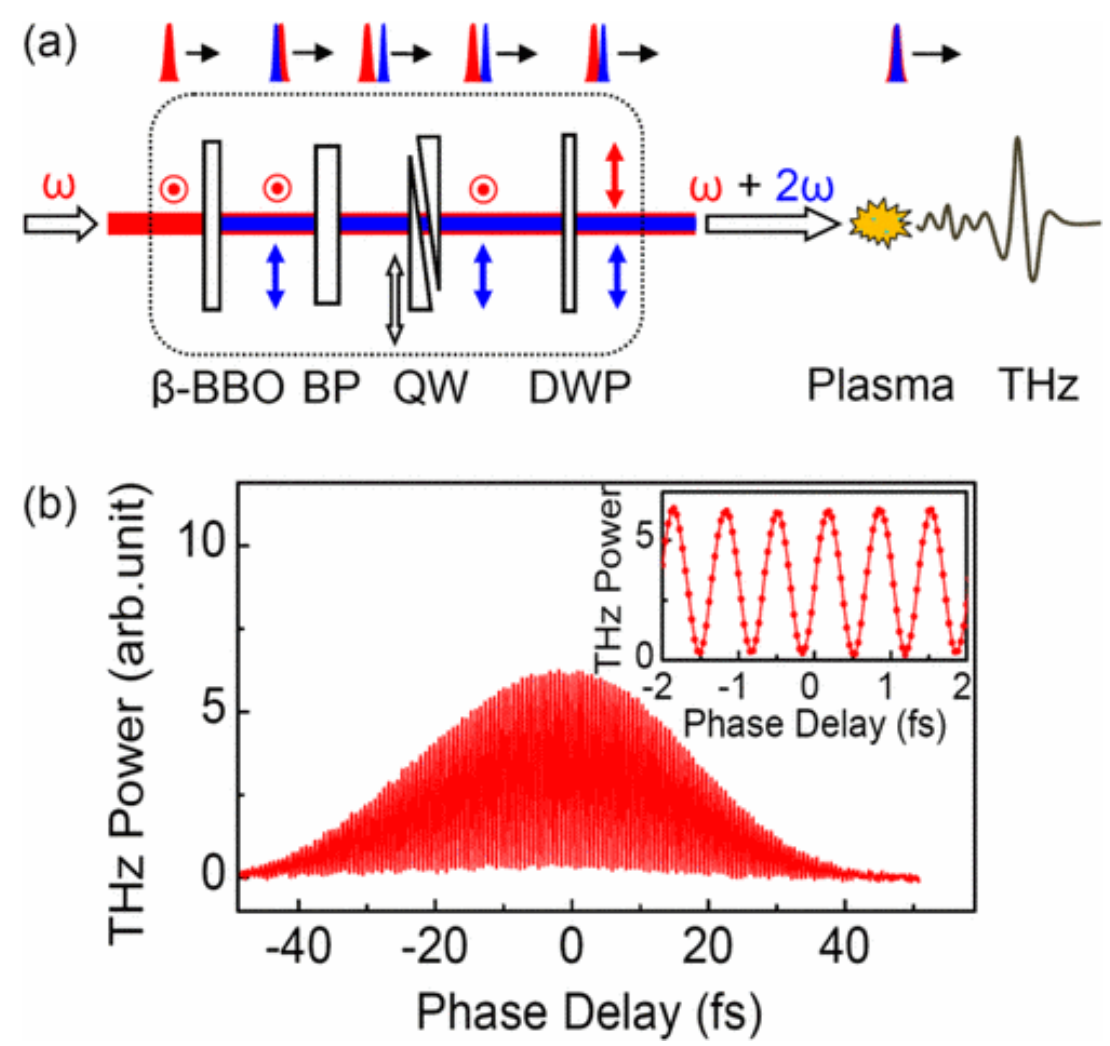

Figure 3.3: Schematic illustration of the experimental setup. Inside the dashed line is the inline phase compensator. $\beta$-BBO, beta barium borate crystal; BP, birefringent plate $(\alpha$-BBO); QW, quartz wedges; DWP, dualwavelength wave plate; the upper and lower arrows near the laser beams indicate the polarization of the $\omega$ and $2 \omega$ beams, respectively. (b) A typical phase curve obtained by changing relative phase between the $\omega$ and $2 \omega$ pulses through the change of the insertion of one of the wedges while monitoring the $\mathrm{THz}$ average power with a pyroelectric detector when the $\omega$ and $2 \omega$ pulses are linearly polarized and parallel to each other; the inset shows a zoomed-in portion of the phase curve. (Reprinted with permission from [7], copyright 2009 American Physical Society) 


\subsection{Experimental Setup of the Two-Color Ex- citation Scheme}

The schematic diagram of the experimental setup is shown in Figure 3.4. A femtosecond amplified Ti: sapphire laser with a central wavelength of 800 $\mathrm{nm}$ and a repetition rate of $1 \mathrm{kHz}$ was used. Unless otherwise stated, the laser pulse duration used in this experiment was 58 fs. A $\beta$-BBO crystal was used for the generation of $2 \omega$ pulses through frequency-doubling, and an in-line phase compensator was applied to accurately control the relative phase between $\omega$ and $2 \omega$ pulses by changing the mechanical insertion of one of the fused silica wedges [7]. A phase compensator was composed of an $\alpha$-BBO crystal, a pair of wedges, and a dual-wavelength wave plate (DWP). The energy of $2 \omega$ pulses was about $10 \%$ of the entire excitation laser energy. Both $\omega$ and $2 \omega$ pulses were vertically polarized after they pass through the phase compensator. Subsequently, $\omega$ and $2 \omega$ laser pulses were co-focused into a $120 \mu \mathrm{m}$ thick water film by a 1-inch effective focal length parabolic mirror to generate $\mathrm{THz}$ waves. The focal point of the laser beam was set to be close to the center of the water film. A liquid jet was employed to obtain the water film. A high-resistivity silicon wafer was used as a filter to block the residual laser beams while allowing the $\mathrm{THz}$ waves to pass through. The $\mathrm{THz}$ electric field was detected by a $3 \mathrm{~mm}$ thick $\langle 110\rangle$-cut ZnTe crystal through EOS [54. Also, the corresponding $\mathrm{THz}$ energy was measured by a commercially available Golay cell with a combination of different filters that eventually 
blocked all the high-frequency components. The angle of incidence on the water film was optimized to be $61^{\circ}$.

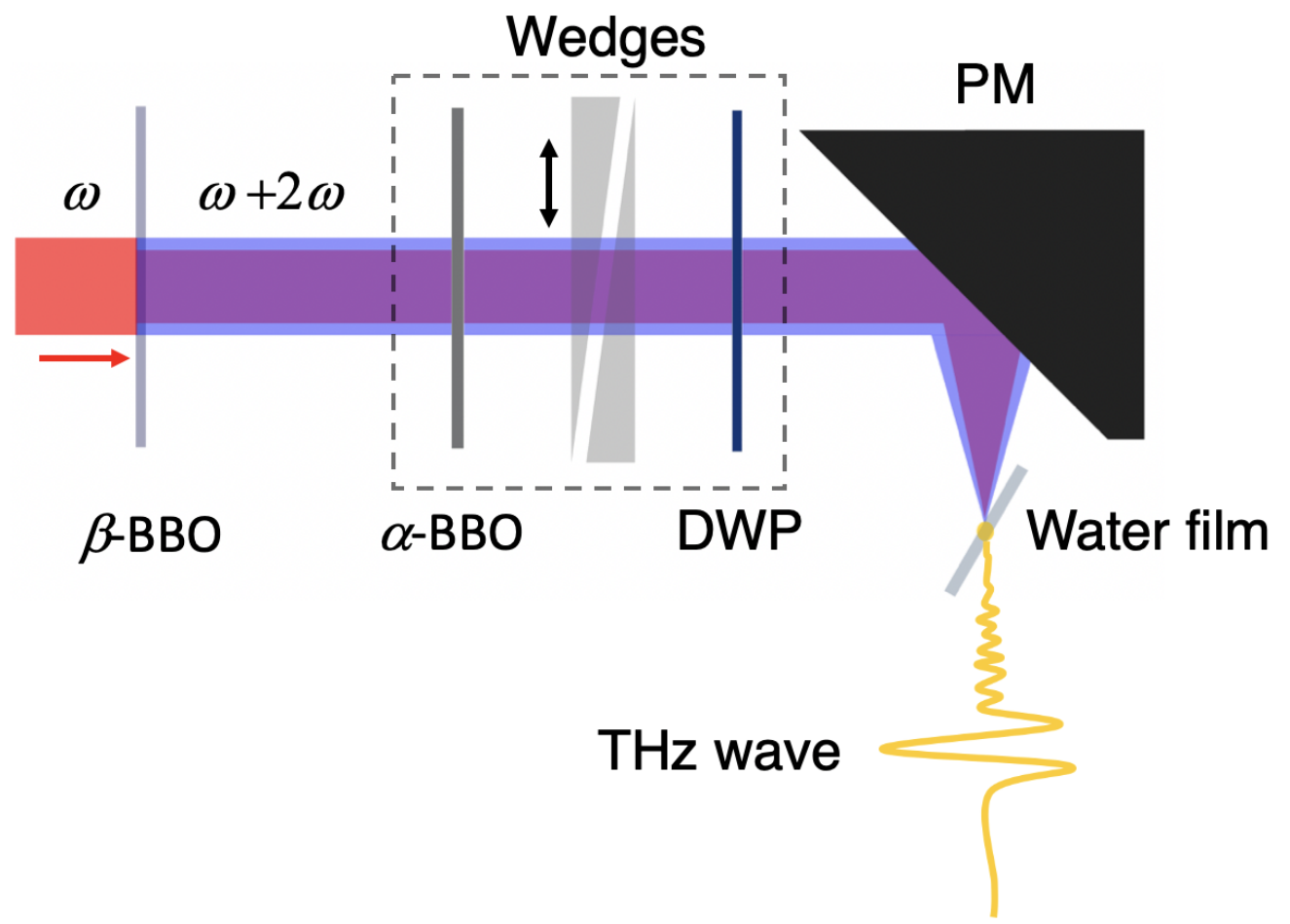

Figure 3.4: Schematic diagram of the experimental setup. A phase compensator composed of an $\alpha$-BBO crystal, a pair of wedges, and a dual-wavelength wave plate (DWP) is applied to control the relative phase between $\omega$ and $2 \omega$ pulses. PM, parabolic mirror with an effective focal length of 1-inch. 


\subsection{Comparison between Terahertz Radiation from One-Color and Two-Color Excitation Scheme}

By using the above experimental setup, $\mathrm{THz}$ emission from liquid water under the two-color excitation scheme is achieved. Remarkably, the $\mathrm{THz}$ electric field generated from the two-color excitation scheme is about 10-times stronger than that from the one-color excitation scheme at the laser pulse duration of $58 \mathrm{fs}$, as shown in Figure 3.5(a). The one-order of magnitude increased $\mathrm{THz}$ electric field is indicative of the two-orders of magnitude enhanced THz energy. The corresponding spectra are shown in Figure 3.5(b). It is noteworthy that the enhancement of the $\mathrm{THz}$ electric field with the asymmetric excitation scheme in water may not be as high as that in air. This could arise from the fact that a short laser pulse duration works well for the case in air, but a longer pulse duration is favored in the ionization process in liquid water, where cascade ionization dominates [50, 74, 95]. More details will be unveiled in Chapter 4 .

Comparatively, experimental results in the case of a longer laser pulse duration (300 fs), which is obtained by chirping the original $58 \mathrm{fs}$ pulse, are shown in Figure 3.5(c) and Figure 3.5(d). The scales of the vertical axis in Figure 3.5(a) and Figure 3.5(c) are the same. Compared to the one-color case, the two-color excitation scheme provides $11 \%$ enhanced $\mathrm{THz}$ electric field when the pulse duration is 300 fs. Such a reduced enhancement rate 

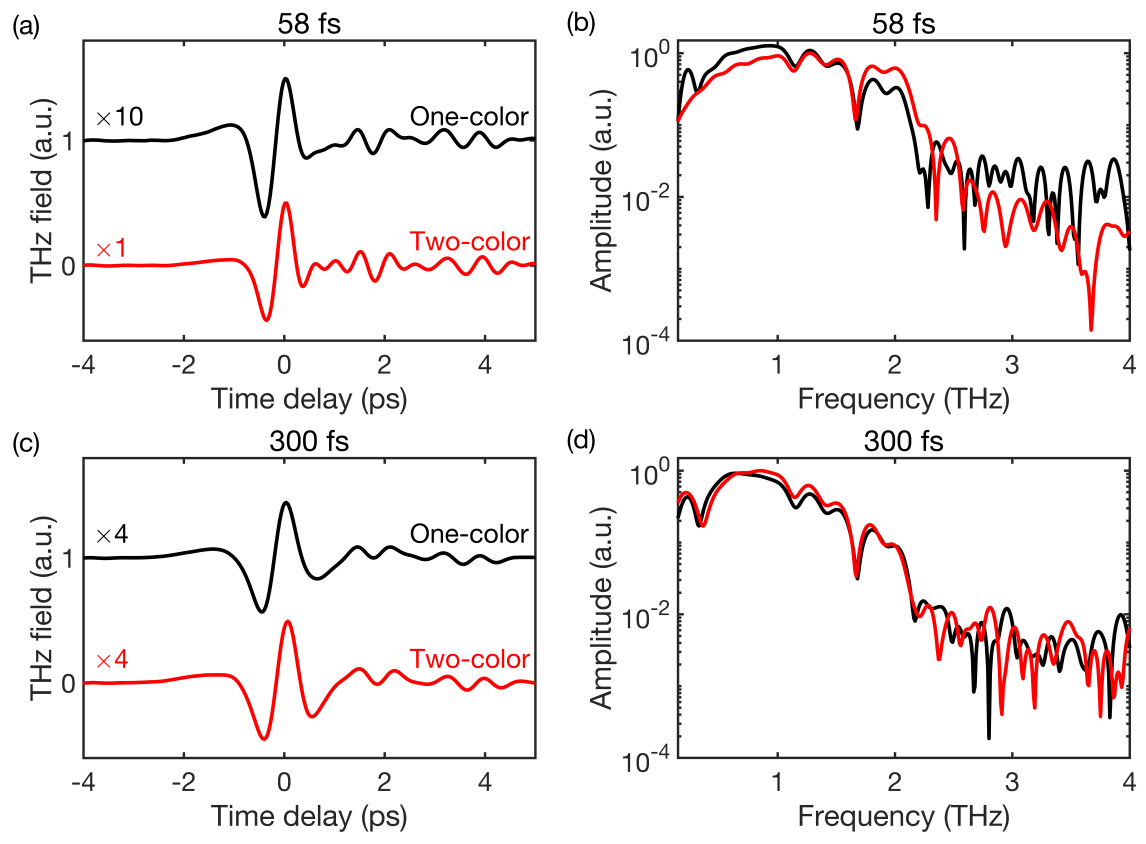

Figure 3.5: Comparison of $\mathrm{THz}$ waves generated from a $120 \mu \mathrm{m}$ thick water film with one-color and two-color excitation schemes. (a), (b) Comparison in the case of a short optical pulse duration (58 fs) in the time domain and frequency domain, respectively. (c), (d) Comparison in the case of a long optical pulse duration (300 fs) in the time domain and frequency domain, respectively. Unified normalization ratios are labeled. 
may be caused by multiple effects. For example, the $\omega$ and $2 \omega$ pulses may have uneven chirps, which reduces the asymmetry of the ionized electron motion and finally decreases the overall generation efficiency of $\mathrm{THz}$ waves. The lower enhancement with a longer pulse duration may also result from the significant drop in second-harmonic generation efficiency as the pulse duration increases. In the experiment, the energy of $2 \omega$ pulse decreases by more than $60 \%$ when the pulse duration increases from 58 fs to 300 fs.

\subsection{Modulation of $\mathrm{THz}$ Fields}

Phenomenologically, the transient photocurrent model can be used to explain the generation process in water and would predict the modulation of $\mathrm{THz}$ fields generated from a water film as well, which is experimentally confirmed, as shown in Figure 3.6. Specifically, Figure 3.6(a) shows that the polarity of the $\mathrm{THz}$ electric field is completely flipped over by changing the relative phase $\phi$ by $\pi$. The inset of Figure 3.6 (a) plots the THz field as a function of optical phase delay between $\omega$ and $2 \omega$ pulses, which indicates that the polarity of the THz electric field is gradually changed with the optical phase delay.

Moreover, an overall phase scan for $\mathrm{THz}$ wave emission from the water film is obtained by gradually adjusting the phase between $\omega$ and $2 \omega$ pulses at an attosecond-level accuracy while monitoring the THz energy with a Golay cell, as shown in Figure 3.6(b). The noise floor is also shown in the figure. 

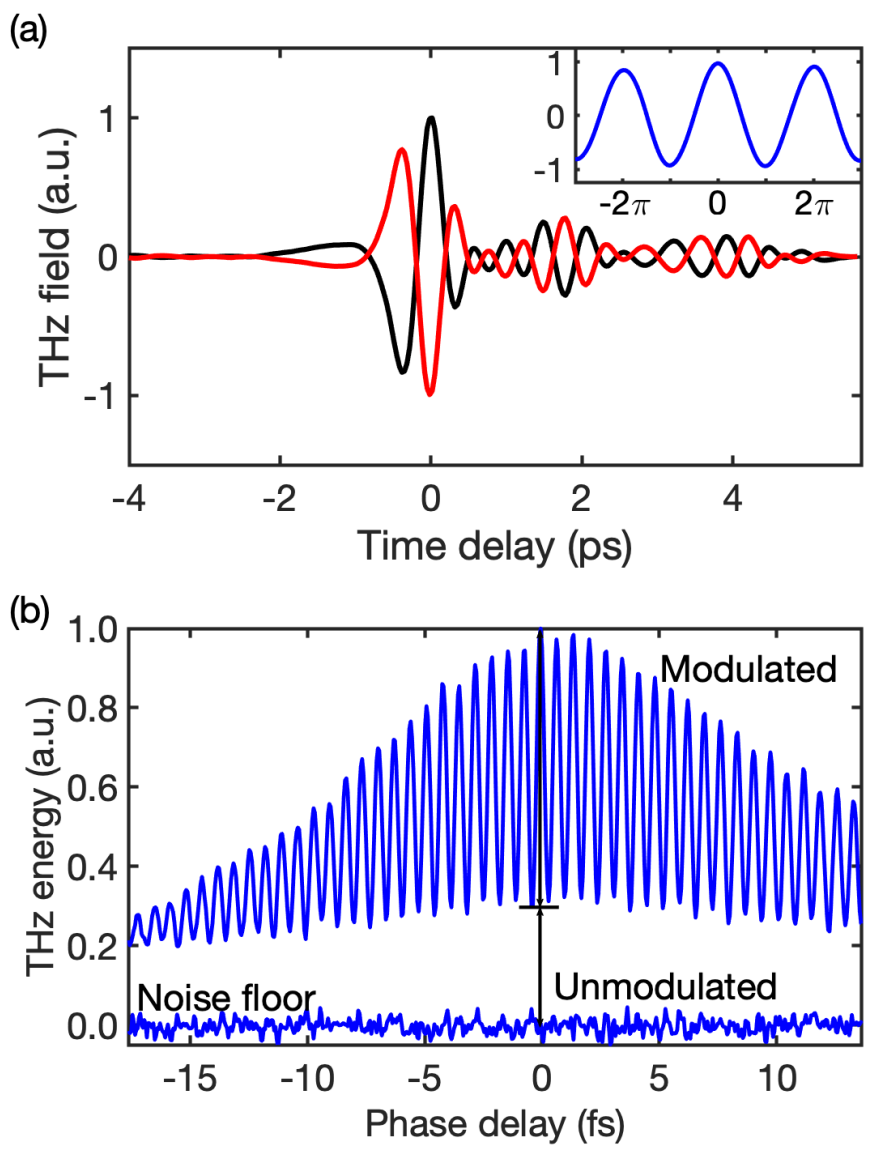

Figure 3.6: Modulation of $\mathrm{THz}$ wave generation from a water film. (a) Comparison of $\mathrm{THz}$ waveforms obtained when the relative phase between $\omega$ and $2 \omega$ pulses is changed by $\pi$ through the change of the insertion of one of the wedges in the phase compensator. Inset, $\mathrm{THz}$ electric field as a function of the phase delay between $\omega$ and $2 \omega$ pulses. (b) An overall phase scan for $\mathrm{THz}$ wave radiation from the water film obtained by gradually changing the phase between $\omega$ and $2 \omega$ pulses while monitoring the THz energy by using a Golay cell. The range of the phase delay is limited by the full length of the wedge. 
The modulated portion shows the phase modulation while the unmodulated portion remains blank at the bottom of the figure. By comparing the energy levels in the figure, the modulated and unmodulated components are estimated to be $70 \%$ and $30 \%$, respectively. It needs to be mentioned that similar modulation can be achieved with a longer optical pulse duration (300 fs) as well.

The modulated and unmodulated $\mathrm{THz}$ waves are related to different generation processes in the plasma. For the further study, the corresponding THz energy varies with the excitation laser pulse energy was measured. Figure 3.7 plots the THz energy as a function of the total excitation pulse ( $\omega$ and $2 \omega$ ) energy. The unmodulated $\mathrm{THz}$ energy (red circles) shows a linear dependence on the laser pulse energy. For the modulated THz energy (blue dots), the modulation does not appear until the excitation pulse energy is beyond $200 \mu \mathrm{J}$. Subsequently, the measurement matches a quadratic fitting above the threshold. In addition, the energy dependence measured from EOS (blue squares) is coincident with the modulated result from the Golay cell.

Similar to the case in air, the modulated THz energy mainly comes from electron acceleration [27, 28, 29, 30] in the transient photocurrent model, while in the full quantum model, the modulated $\mathrm{THz}$ radiation may also result from the buildup of bremsstrahlung from electron-atom collisions [30]. In contrast, the unmodulated $\mathrm{THz}$ energy may arise from multiple physical processes. For instance, a spatial net charge distribution created by the ponderomotive force radiates $\mathrm{THz}$ waves [23]. Since no threshold is observed for 


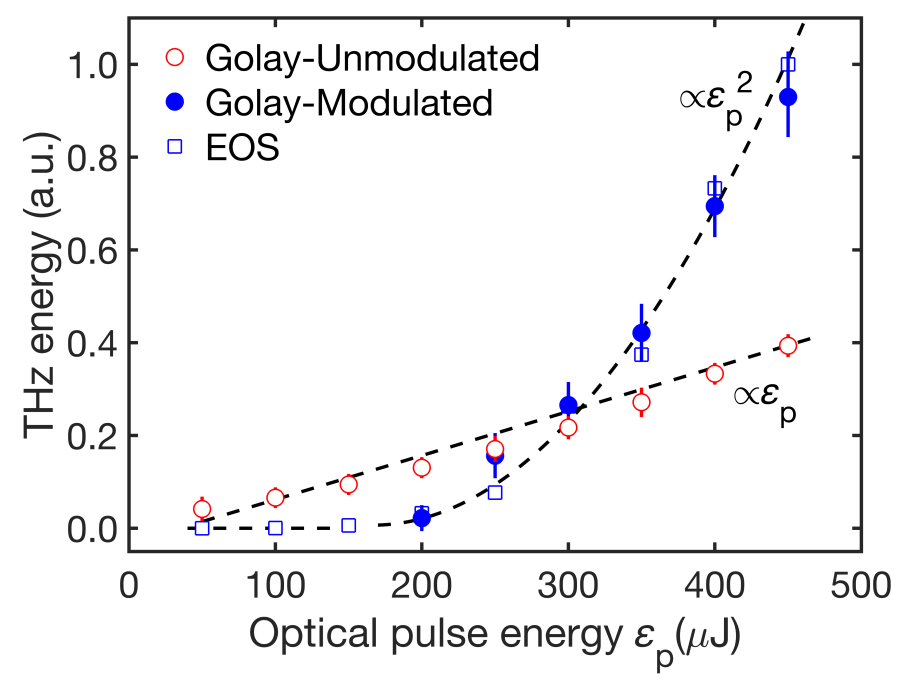

Figure 3.7: Normalized $\mathrm{THz}$ energy from liquid water as a function of the total excitation optical pulse $(\omega$ and $2 \omega$ ) energy. Blue squares, THz energy calculated from the temporal integral of the $\mathrm{THz}$ waveform measured by EOS. Blue dots, modulated THz energy measured by the Golay cell. Red circles, unmodulated THz energy measured by the Golay cell. The maximum pulse energy is limited by the available laser pulse energy in the experiment. 
the unmodulated portion, the $\mathrm{THz}$ wave emission can be attributed to part of the broadband radiation from the combination of thermal bremsstrahlung from electrons and electron-ion recombination [51]. Moreover, the energy dependence in Figure 3.7 indicates that the ratio of the modulated $\mathrm{THz}$ energy to unmodulated THz energy increases with the laser pulse energy. The unmodulated component is stronger with weak excitation pulses while the modulated component will dominate if intense laser pulses are used.

In consideration of the fact that the liquid source can quickly replenish itself due to its fluidity, the $\mathrm{THz}$ wave emission can be dramatically scaled up by increasing the excitation laser energy, which reveals liquid water's potentiality to emit intense THz waves. Although no saturation occurs in Figure 3.7 when the laser pulse energy is up to $450 \mu \mathrm{J}$, it is worth mentioning that the saturation should be observed when the excitation energy is sufficiently high. The measured THz electric field strength is estimated as $1.1 \mathrm{kV} / \mathrm{cm}$ when the excitation laser pulse energy is $450 \mu \mathrm{J}$. Realistically, the measured value is much weaker than the generated $\mathrm{THz}$ radiation due to the absorption of the water film itself and total internal reflection on the water-air boundary, etc. 


\subsection{Discussion about Contributions from Wa- ter and Air}

When the optical beam is focused into the water film, it will also possibly create air plasmas located close to the air-water interface and water-air interface. Thus, discussions are necessary to address the contributions from those air plasmas. Actually, the experiment in Section 2.3 has already been carried out to ensure that $\mathrm{THz}$ waves generated with this focusing geometry are primarily from the plasmas located inside the water film. In this experiment of two-color excitation, the focusing geometry is the same as that used in Section 2.3. The maximum excitation laser pulse energy $450 \mu \mathrm{J}$ is similar to that $(400 \mu \mathrm{J})$ used in Section 2.3 . Moreover, the larger angle of incidence onto the water film increases the laser path length within the water film, which also helps to hold the majority of the plasmas inside the water film.

To further compare the $\mathrm{THz}$ wave emission from the water film (at the focus) and that from the air plasma (at the interface) in the experiment, the ratios of the corresponding THz electric field strength $E_{a}\left(z_{i}\right) / E_{w}\left(z_{0}\right)$ and $\mathrm{THz}$ power $P_{a}\left(z_{i}\right) / P_{w}\left(z_{0}\right)$ can be estimated. The subscript $w$ represents water and $a$ represents air. $z_{0}$ and $z_{i}$ are the locations at the focus and the interface, respectively. It is assumed that no water is present when we estimate the values of $E_{a}$ and $P_{a}$. The influence of the water film will be discussed later. Both the laser beam and the generated $\mathrm{THz}$ beam are assumed to have a Gaussian profile for this approximation. 
Under the experimental condition, the laser intensity in the air at the focus is assumed to be $I\left(z_{0}\right)=1 \times 10^{15} \mathrm{~W} / \mathrm{cm}^{2}$ [96, 97]. The laser intensity $I\left(z_{i}\right)$ at the interface is calculated through $I\left(z_{i}\right)=I\left(z_{0}\right)\left[w\left(z_{0}\right) / w\left(z_{i}\right)\right]^{2}$, where $w\left(z_{0}\right)$ and $w\left(z_{i}\right)$ are the radii of the laser beam at the focus and the interface, respectively. Thus, the ratio of generated $\mathrm{THz}$ electric field strength from the air at the focus to that at the interface $E_{a}\left(z_{i}\right) / E_{a}\left(z_{0}\right)$ is calculated by the transient photocurrent model [27, 28, 29]. By experimentally measuring $E_{a}\left(z_{0}\right)$ and $E_{w}\left(z_{0}\right)$, the ratio $E_{a}\left(z_{i}\right) / E_{w}\left(z_{0}\right)=E_{a}\left(z_{i}\right) / E_{a}\left(z_{0}\right) \times$ $E_{a}\left(z_{0}\right) / E_{w}\left(z_{0}\right)=0.05 \%$ is obtained. Then, the ratio of the $\mathrm{THz}$ intensity $I_{a}\left(z_{i}\right) / I_{w}\left(z_{0}\right)$ is calculated from $E_{a}\left(z_{i}\right) / E_{w}\left(z_{0}\right)$. Finally, the ratio of the $\mathrm{THz}$ power $P_{a}\left(z_{i}\right) / P_{w}\left(z_{0}\right)=0.02 \%$ can be obtained by integrating the corresponding $\mathrm{THz}$ intensity over its cross-section.

Consequently, the $\mathrm{THz}$ wave radiation from the air at the interface is negligible, in comparison to the contribution from the water film. In fact, the measurable THz field from the air plasmas at the interfaces should be even smaller. Specifically, the water film will either absorb $83 \%$ of the THz energy from the plasma located close to the first interface (air-water) or decrease the laser intensity by affecting the focusing geometry for the plasma near the second interface (water-air). Based on the above discussion, $\mathrm{THz}$ waves generated in the experiment are mainly attributed to the plasma within the water film rather than the air plasma. 


\subsection{Summary}

In this chapter, $\mathrm{THz}$ emission from a water film under the two-color excitation scheme is investigated. The generated $\mathrm{THz}$ field is about one-order of magnitude stronger than that with the one-color excitation scheme when short laser pulses are used. The enhancement of $\mathrm{THz}$ radiation with the asymmetric excitation scheme in water is not as high as that in air. Additionally, the modulation of $\mathrm{THz}$ waves generated from liquid water with an asymmetric excitation scheme is achieved by adjusting the relative phase between $\omega$ and $2 \omega$ pulses. The polarity of the THz electric field is completely flipped over by changing the relative phase $\phi$ by $\pi$. The method also facilitates the observation of modulated and unmodulated $\mathrm{THz}$ waves with a Golay cell. By increasing the excitation laser pulse energy, the unmodulated component is linearly raised while the modulated component is quadratically enhanced above the threshold, which indicates that the $\mathrm{THz}$ wave emission can be dramatically scaled up by increasing the excitation optical pulse energy. 


\section{Chapter 4}

\section{Terahertz Wave Generation from a Wa- ter Line}

In this chapter, the use of water lines in place of water films as $\mathrm{THz}$ emitters to obtain stronger $\mathrm{THz}$ signals is proposed and demonstrated. Such a geometry of the emitter mitigates the total internal reflection at the flat water-air interface, and thus more $\mathrm{THz}$ radiation is coupled out. Owing to the availability of water lines with different diameters, optimal pulse duration and optimal water line's diameter are investigated.

\subsection{Issues of Using Water Films}

It was mentioned in Chapter 2 that a wire-guided free-flowing thin water film was developed to emit $\mathrm{THz}$ waves under the excitation of laser pulses. The observation shows that the film will be broken if high-energy laser pulses are focused onto it, therefore, the generation of intense $\mathrm{THz}$ radiation is restrained. To overcome this issue, a nozzle jet was used to produce a forced- 
flowing water film to bear intense laser pulses. Such an arrangement significantly increases the THz energy under intense optical excitation. However, a common problem occurred with the geometry of a film is that the majority of generated THz waves cannot be coupled out from the water film. Specifically, the $\mathrm{THz}$ signals are strongly absorbed by the millimeter-width water in the side-way directions. Aside from the absorption of water, the total internal reflection at the flat water-air interface will tremendously reduce the $\mathrm{THz}$ signals (see Figure 2.12). Furthermore, due to the limitation in the fixed dimension of nozzle jets, water films with various thicknesses in a large range are not easily attainable, which impedes the systematical investigation on the optimal conditions for $\mathrm{THz}$ wave generation from liquid water. In order to ameliorate these drawbacks associated with using water films, water lines are introduced as the THz source.

\subsection{Water Lines}

Figure 4.1 shows a series of syringe needles $\left(\right.$ BSTEAN $\left.^{\mathrm{TM}}\right)$ using to produce water lines. Inner diameters of the available syringe needles range from 0.06 $\mathrm{mm}$ to $1.65 \mathrm{~mm}$. The gauge number/inner diameter of a syringe needle is represented by its color. A Masterflex L/S Digital Pump from Cole-Parmer ${ }^{\circledR}$ was utilized to drive the water to form the water line flowing along the $y$ direction.

Figure 4.2 is a photo of the water line produced by a syringe needle of 


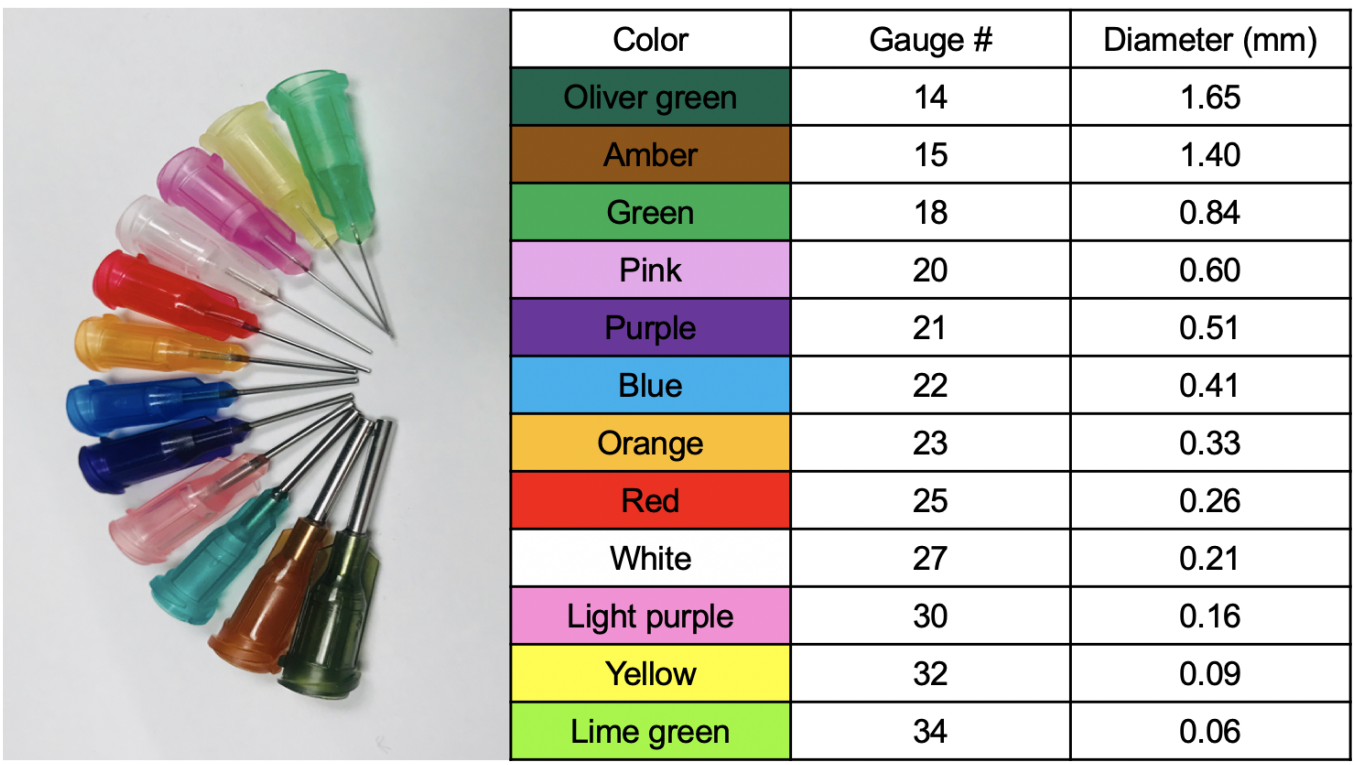

Figure 4.1: Photograph of syringe needles. The color of a syringe needle indicates its gauge number and inner diameter.

$260 \mu \mathrm{m}$ inner diameter. It is experimentally confirmed that the diameter of the top part of the water line is identical to the inner diameter of the syringe needle.

A femtosecond amplified Ti: sapphire laser with a central wavelength of $800 \mathrm{~nm}$ and a repetition rate of $1 \mathrm{kHz}$ was used for the excitation. The flow velocity of the water line was controlled to be $7 \mathrm{~m} / \mathrm{s}$. In this case, each laser pulse interacted with a fresh water spot. The horizontally polarized laser beam with $0.4 \mathrm{~mJ}$ pulse energy propagating along the $z$-direction was focused into the water line by a 2-inch effective focal length lens $(\mathrm{F} / 4)$ to generate $\mathrm{THz}$ waves. A high-resistivity silicon wafer acted as a filter to block the residual laser beam while allowing the $\mathrm{THz}$ beam passing through. The 


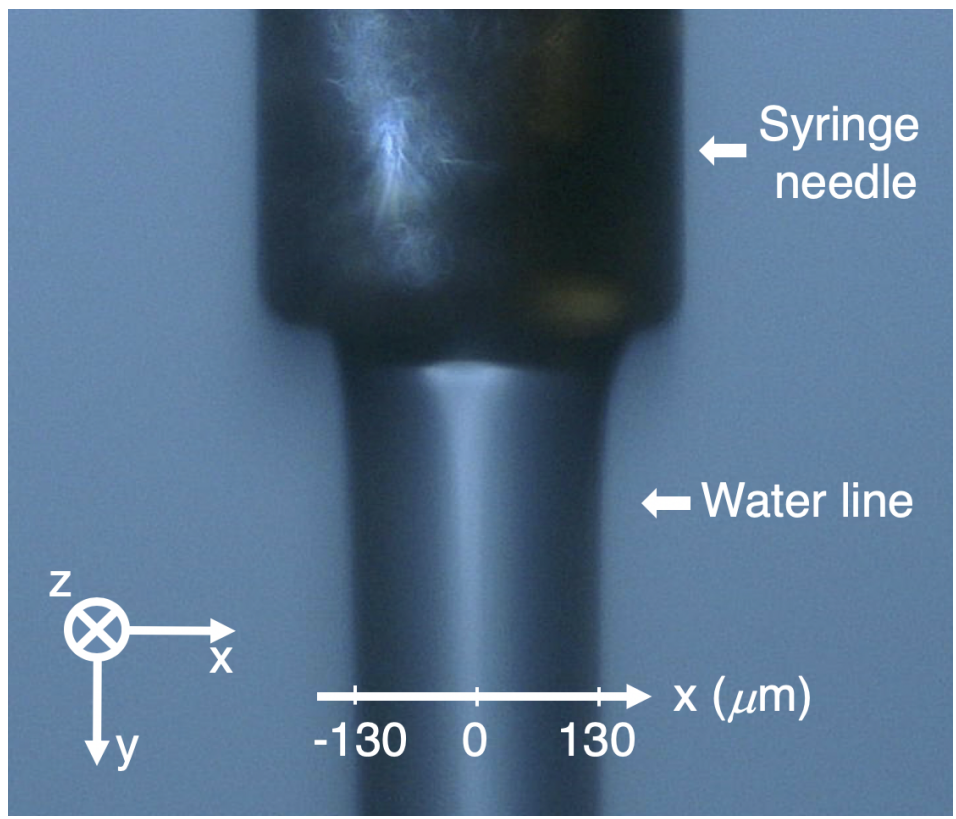

Figure 4.2: Photograph of the water line produced by a syringe needle with $260 \mu \mathrm{m}$ inner diameter. The diameter of the water line is $260 \mu \mathrm{m}$ as well. The flow velocity is about $7 \mathrm{~m} / \mathrm{s}$ along the $y$-direction. The laser beam propagates in the $z$-direction. The water line can be moved along the $x$-direction by a translation stage. 
THz electric field was measured by a $2 \mathrm{~mm}$ thick $\langle 110\rangle$-cut ZnTe crystal placed in the direction of laser propagation through EOS [54].

\section{3 $\mathrm{THz}$ Radiation from the Water Line}

Figure 4.3(a) shows the peak values of THz fields when the $260 \mu \mathrm{m}$ diameter water line is scanned along the $x$-direction across the laser focal point. A weak THz signal is detected at $x=0 \mu \mathrm{m}$, which is represented as the black dot in the middle. This coincides with the case of a water film with a normally incident laser beam (see Figure 2.17). By contrast, the THz signal becomes significant when the water line is shifted away from the zero position in the $x$-direction. The THz field is maximized at $x= \pm 90 \mu \mathrm{m}$, which is caused by the ponderomotive force-induced current with the symmetry broken around the interface [55]. The value of the $\mathrm{THz}$ peak field changes in sign from negative (red dots) to positive (blue dots) when the $x$ position varies from negative to positive. If two points are symmetric about 0 , their absolute values of $\mathrm{THz}$ peak field are identical.

The waveforms of the THz signals at $x= \pm 90 \mu \mathrm{m}$ are shown in Figure 4.3(b). Clearly, the THz waveforms flip over when their $x$ positions are mirrored with respect to the zero position. The results in Figure 4.3(a) and Figure 4.3(b) can also be understood from the dipole radiation model proposed in Section 2.8: mirrored $x$ position leads to an opposite projected direction of the dipole in water, resulting in an inverted $\mathrm{THz}$ waveform. 

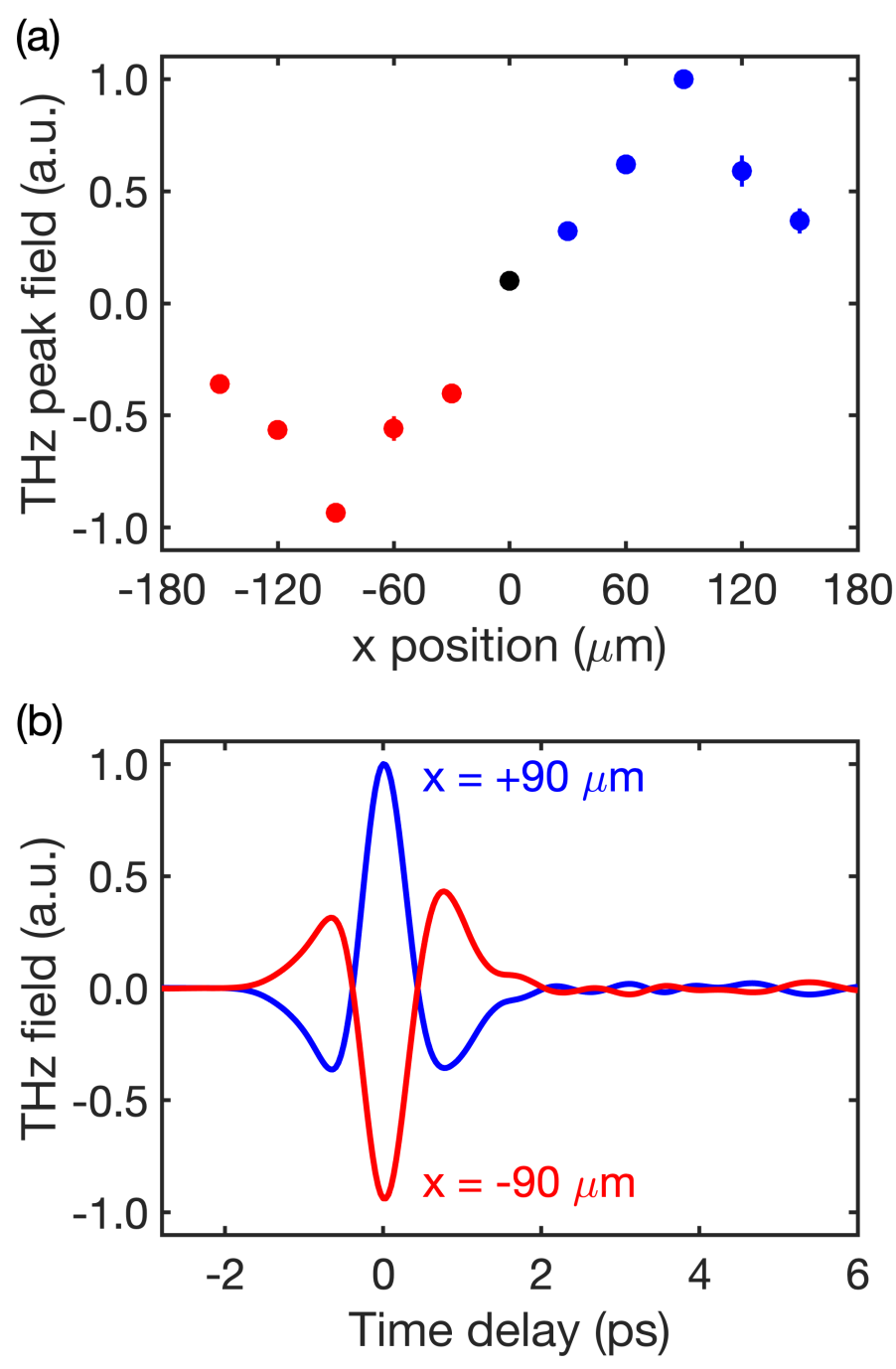

Figure 4.3: (a) THz peak fields with different $x$ positions when the $260 \mu \mathrm{m}$ diameter water line is crossing the laser focal point along the $x$-direction. (b) $\mathrm{THz}$ waveforms of $x= \pm 90 \mu \mathrm{m}$ in (a). 
For $x=0 \mu \mathrm{m}$, the dipole in water is oriented along the direction of laser propagation contributing to weak $\mathrm{THz}$ radiation in the $z$-direction. Thus, a tilted dipole realized by a shift of the water line in the $x$-direction is essential to obtain the strongest $\mathrm{THz}$ signal in the direction of laser propagation. Note that unlike the case of a water film, $\mathrm{THz}$ signals generated from a water line can also be coupled out and detected in the $x$-direction. This will increase the overall coupling of the $\mathrm{THz}$ radiation.

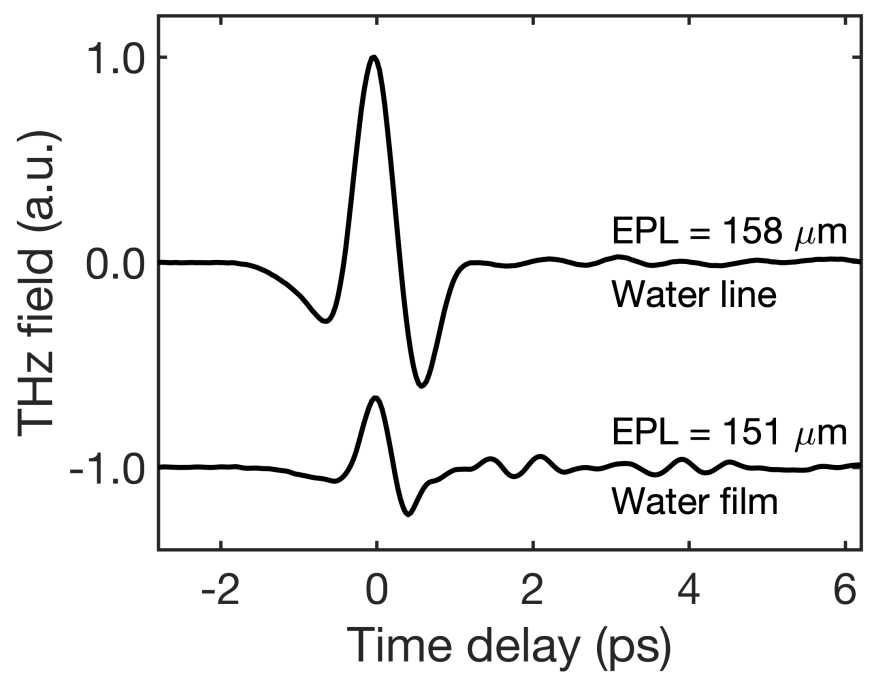

Figure 4.4: Comparison of $\mathrm{THz}$ fields generated from a $210 \mu \mathrm{m}$ water line and a $120 \mu \mathrm{m}$ water film. EPL, effective path length. A shift of the $210 \mu \mathrm{m}$ water line in the $x$-direction makes the EPL to be $158 \mu \mathrm{m}$. Oblique incidence on the $120 \mu \mathrm{m}$ water film leads to a $151 \mu \mathrm{m}$ EPL.

To make a reasonable comparison between the $\mathrm{THz}$ waves generated from a water line and a water film, a $210 \mu \mathrm{m}$ diameter water line and a $120 \mu \mathrm{m}$ thick water film were used to assure that their optical effective path lengths 
(EPLs) in water were similar. The incident angle on the water film is $60^{\circ}$, which leads to the $158 \mu \mathrm{m}$ EPL in the film. The shift of the water line in the $x$-direction causes the EPL to be $151 \mu \mathrm{m}$. Figure 4.4 plots the THz waveforms generated from the two cases. 2.8-times enhanced THz electric field (peak-valley) is obtained by using the water line to substitute the water film. Compared with the flat water-air interface of a film, the curved interface of a water line helps to reduce the total internal reflection of the $\mathrm{THz}$ waves, and therefore, more THz radiation is coupled out and detected.

\subsection{Preference for Sub-Picosecond Laser Pulses}

It was shown in Section 2.10 that optically induced $\mathrm{THz}$ wave generation from air plasma generally requires a short temporal laser pulse. In contrast, $\mathrm{THz}$ radiation from water prefers a longer pulse, wherein the mechanism remains unclear.

Additionally, it is noticeable that the thicknesses of the water film used in Section 2.2 and Section 2.7 are $178 \mu \mathrm{m}$ and $120 \mu \mathrm{m}$, respectively. The laser pulse durations are individually optimized as $550 \mathrm{fs}$ and $300 \mathrm{fs}$ in the above two cases. This phenomenon reveals speculation that optimal laser pulse duration depends on the EPL in water. The data from a series of different water thicknesses are indispensable to draw a solid conclusion. Benefited from the availability of a series of syringe needles with various sizes, water lines with diameters varied from tens of microns to a few millimeters are 
realized, which offer the opportunity for a thorough investigation into the influence of optical pulse duration on $\mathrm{THz}$ wave generation from liquid water.

To explore the dependence of $\mathrm{THz}$ radiation on the optical pulse duration, laser pulses were stretched in time to achieve various pulse durations $\tau_{\mathrm{p}}$. Corresponding $\mathrm{THz}$ energy from a $210 \mu \mathrm{m}$ diameter water line was recorded and shown as the black dot in Figure 4.5. Unlike the case of air, the optimization for $\mathrm{THz}$ radiation from liquid water prefers a sub-picosecond laser pulse (345 fs). In this dissertation, "sub-picosecond" is defined as 200-800 fs.

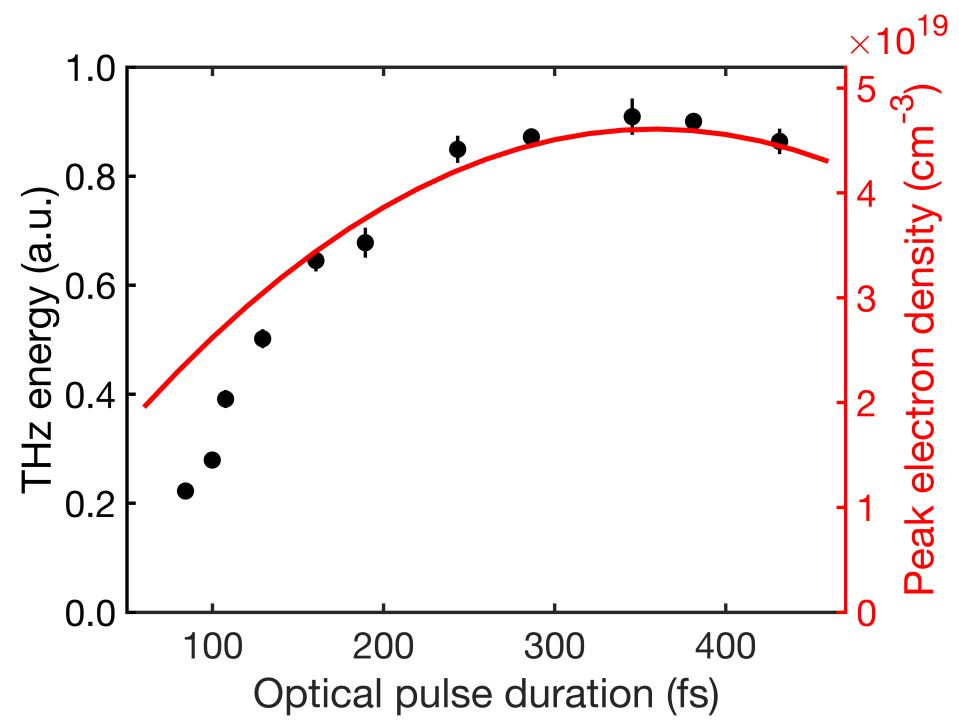

Figure 4.5: Effect of optical pulse duration on THz energy and peak electron density for a $210 \mu \mathrm{m}$ water line. The black dots are the experimental data for $\mathrm{THz}$ energy. The red curve is the simulation data for peak electron density.

The result of the optimal pulse duration can be understood from the interaction between the laser field and the water, as described by Equation 2.2 and Equation 2.3. Electrons are produced by intense laser pulses through 
$\mathrm{MPI} / \mathrm{TI}$ and cascade ionization at the focus. It is worth underlining that each cascade process needs an ionization time $K \tau$ [74] to perform. Thus, one seed electron cannot produce more than $2^{\tau_{\mathrm{p}} / K \tau}$ electrons through the cascade process, regardless of the laser intensity. Compared with the increase in the number of electrons due to MPI/TI, the exponential boost caused by cascade ionization makes it the dominating ionization process in water when $\tau_{\mathrm{p}}$ is above $40 \mathrm{fs}$ [74]. Therefore, a longer pulse duration that allows more cascades taking place benefits plasma formation through the exponential increase from cascade ionization. Even though the majority of electrons are provided by cascade ionization, MPI/TI is still quite important because it provides most of the electrons to initiate the cascade process, especially in pure water. Since MPI/TI highly depends on laser intensity and laser intensity is inversely proportional to $\tau_{\mathrm{p}}$ when the laser pulse energy $\varepsilon_{\mathrm{p}}$ is fixed (see Equation 2.4 , generated electron density will start to decrease if $\tau_{\mathrm{p}}$ raises too much. Considering the fact that higher electron density contributes to more $\mathrm{THz}$ radiation, the trade-off between the two effects can lead to the preference for sub-picosecond pulses.

Simulations that based on Equation 2.2 and Equation 2.3 are carried out to verify the theoretical analysis. The laser wavelength and pulse energy are set to match the values in the experiment (see Section 4.2). The laser intensity at the focus is calculated to be at the level of $10^{15} \mathrm{~W} / \mathrm{cm}^{2}$. In such a case, the Keldysh parameter $\gamma=0.2$ implies that TI dominates over MPI. In the experiment, a strong external focusing is used, where the SF 
and the geometrical focusing become indistinguishable [98]. Thus, the term of $\mathrm{SF}$ is not included in the simulation. The impact of GVD is assumed to be insignificant because the plasma length of our interest is short $(\sim 200$ $\mu \mathrm{m})$. A commonly used value of electron collision time $\tau=1 \mathrm{fs}$ is applied [74. Water is treated as an amorphous semiconductor with a bandgap of $E_{\mathrm{g}}=6.5 \mathrm{eV}$. Since femtosecond and sub-picosecond laser pulses are used in the experiment, contribution from diffusion is justifiably neglected [74, 77]. The TI ionization rate $w$ and the cascade ionization rate $\eta_{\text {cas }}$ are calculated by Equation 2.6 and Equation 2.7 respectively. The recombination rate $\eta_{\text {rec }}$ is taken to be $2 \times 10^{-9} \mathrm{~cm}^{3} / \mathrm{s}[74,99$. The molecular density of water is $3.34 \times 10^{22} \mathrm{~cm}^{-3}$ [50]. The initial electron density is chosen to be $\rho_{0}=$ $10^{10} \mathrm{~cm}^{-3}$, which will hardly impact on the evolution of electron density even if $\rho_{0}$ is changed over several orders of magnitude [77, 100]. By numerically solving [77] the coupled Equation 2.2 and Equation 2.3 with different values of pulse duration, the evolution of electron density $\rho(z, t)$ that corresponds to different $\tau_{\mathrm{p}}$ is obtained. Consequently, the peak electron density versus $\tau_{\mathrm{p}}$ is achieved and plotted as the red curve in Figure 4.5. As expected, a sub-picosecond pulse is a requisite for the highest peak electron density.

For further verification, the diameter of the water line $d$ is chosen as a variable to see how the optimal pulse duration changes with the water line diameter in the experiment and simulation. Experimentally, seven individual syringe needles with different inner diameters were used to produce water lines with diameters varying from tens of microns to half a millimeter. The 


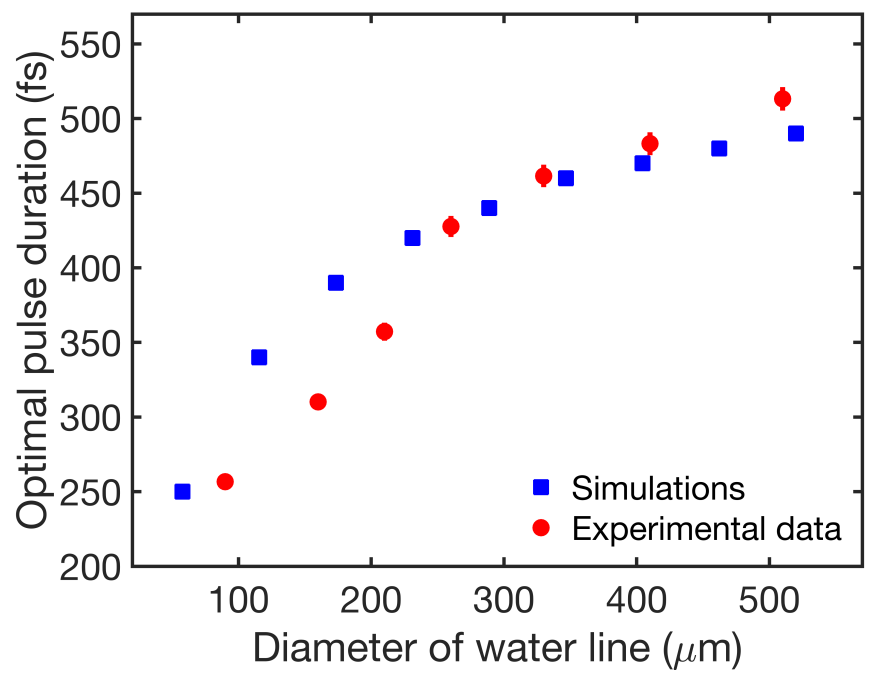

Figure 4.6: Optimal optical pulse duration versus the diameter of the water line. The blue squares are simulations of optimal pulse duration aiming for the highest electron density. The red dots are the experimental data of optimal pulse duration obtained with the strongest $\mathrm{THz}$ energy. 
optimal pulse duration for the THz energy from each water line was recorded and shown as the red dot in Figure 4.6. The optimal pulse duration gradually changes from 257 fs to 513 fs when $d$ increases from $90 \mu \mathrm{m}$ to $510 \mu \mathrm{m}$. In the simulation, the evolution of electron density that corresponds to different $\tau_{\mathrm{p}}$ is achieved. Then the peak electron density is integrated over $z$ within the whole range of the water line that is determined by the diameter $d$. Thus, the optimal pulse duration for the highest electron density that corresponds to the different diameter of the water line is obtained (see the blue squares) in Figure 4.6. To make the result clear to see, only a few points in a similar range of the experiment are shown. The coincidence of the experimental result and the simulation supports the model and the explanation.

The corresponding $\mathrm{THz}$ energy from each water line is plotted in Figure 4.7. The maximum $\mathrm{THz}$ energy is obtained with a $210 \mu \mathrm{m}$ diameter water line, which results from the trade-off between laser-water interaction length and absorption of water for $\mathrm{THz}$ waves. Specifically, a very thin water line does not have sufficient length for the interaction between laser pulses and water molecules while a very thick water line suffers from serious absorption. Neither case does offer strong $\mathrm{THz}$ radiation. Note that the $\mathrm{THz}$ energy slightly decreases rather than significantly drops when the diameter of the water line increases from $330 \mu \mathrm{m}$ to $510 \mu \mathrm{m}$. The reason is that during the optimization process for the maximal $\mathrm{THz}$ signal, the water line was moved toward the incoming laser beam along the $z$-direction to compensate for the absorption of water. Consequently, the laser focal point was close 
to the second interface (water-air interface) instead of being located at the center of the water line. No increment of the THz energy is observed when the water line's diameter raises from $330 \mu \mathrm{m}$ to $510 \mu \mathrm{m}$, which reveals that further extension of the interaction length no longer helps.

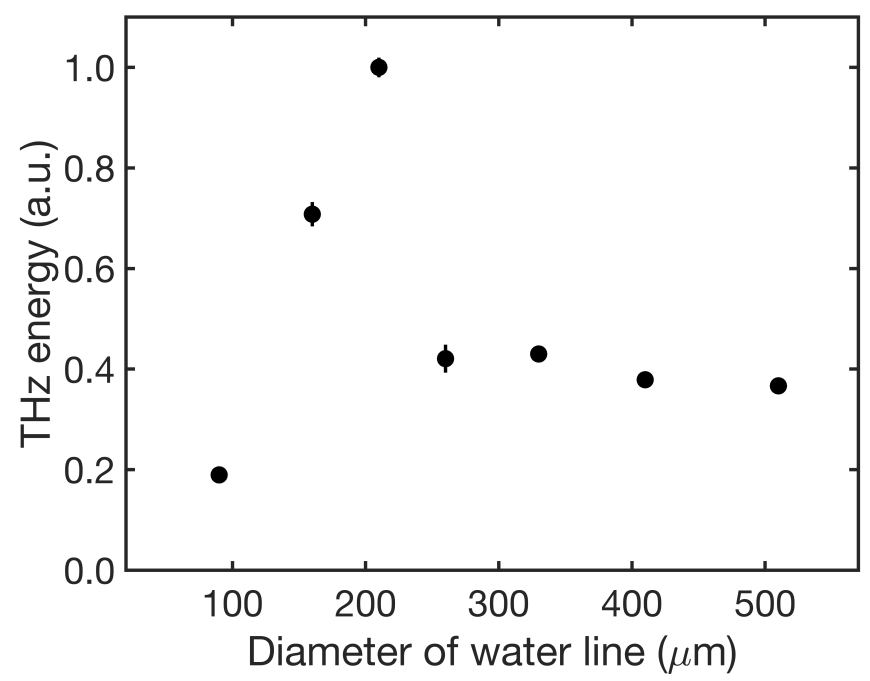

Figure 4.7: THz energy versus the diameter of the water line.

It needs to be mentioned that the demonstration of the preference for sub-picosecond optical pulses can also be used to explain the low enhancement of the $\mathrm{THz}$ radiation under the two-color excitation in Section 3.5 , Sub-picosecond pulses are indispensable to achieve high electron density in water. Nevertheless, a short pulse works much better for the second-harmonic generation from the fundamental beam. The discrepant demand regarding the pulse duration significantly limits the generation of $\mathrm{THz}$ waves under the two-color excitation. Therefore, an intense $\mathrm{THz}$ radiation could be ex- 
pected if the requirements for pulse duration are satisfied under the two-color excitation. One possible solution is separately controlling the phase and polarization [101] of the fundamental beam and the second-harmonic beam by using a dichroic mirror. Another dichroic mirror can be applied to combine the second-harmonic beam and the stretched fundamental beam. Such an arrangement assures that the plasma would be generated by a time-stretched pulse and the second harmonic component remains intense.

\subsection{Summary}

Water lines produced by syringe needles generate stronger $\mathrm{THz}$ radiation by mitigating the total internal reflection at the water-air interface than water films do. It is anticipated that the overall coupling is further increased because $\mathrm{THz}$ signals can also be coupled out in the $x$-direction.

By now, the majority of observations in $\mathrm{THz}$ wave generation from water [95, 102, 103 resembles those from the air because laser-induced plasma formation plays a crucial role in both generation processes. In the two cases, the model based on a ponderomotive force-induced dipole was commonly used for the one-color excitation scheme [23, 24, 55, 102] and the transient current model was successfully applied for the two-color excitation scheme [27, 28, 29, 103]. Remarkably, one distinction between the two cases is that the dependence of $\mathrm{THz}$ radiation on the optical pulse duration is very different: $\mathrm{THz}$ radiation from gases favors a short optical pulse while a longer pulse 
offers stronger THz emission in liquids. The availability of a series of different sizes of water lines enables a thorough investigation into the preference of such a sub-picosecond pulse.

The preference for sub-picosecond laser pulses for $\mathrm{THz}$ wave generation from liquids is understood from the process of laser-induced plasma formation based on the experimental results and simulations. On one hand, cascade ionization, the dominant ionization process leading to an exponential increase in the number of electrons, needs a long pulse duration. On the other hand, increasing the pulse duration sacrifices the laser intensity, which limits the generation of plasmas in the liquid. The trade-off between the two effects results in an optimal pulse duration around the sub-picosecond region.

Moreover, it is found that the optimal pulse duration highly depends upon the diameter of the water line, which also impacts on the $\mathrm{THz}$ radiation energy. The maximum $\mathrm{THz}$ signal is obtained with a $210 \mu \mathrm{m}$ diameter water line in the experiment. 


\section{Chapter 5}

\section{Terahertz Wave Generation from $\alpha$-Pinene}

In this chapter, $\alpha$-Pinene is introduced to replace water as the $\mathrm{THz}$ emitter because it almost does not absorb $\mathrm{THz}$ waves. Compared with water, $\alpha$ Pinene generates a higher $\mathrm{THz}$ electric field and provides a wider bandwidth.

\section{$5.1 \alpha$-Pinene}

In previous sections, the strong absorption of liquid water and its impacts on the $\mathrm{THz}$ signals (see Equation 2.8 have been discussed. To make sure that there are enough $\mathrm{THz}$ waves can be coupled out from the generation site for the detection, a thin water film or water line has been utilized as the $\mathrm{THz}$ emitter. Another approach to avoid suffering from the strong absorption is to use a liquid with weaker absorption in the $\mathrm{THz}$ frequency regime as the source.

$\alpha$-Pinene is a popular non-polar liquid, which can be found in the oils

of the pine. It is an organic compound of the terpene class. Nontoxic and 
colorless $\alpha$-Pinene makes it a good candidate for the $\mathrm{THz}$ emitter. Before using $\alpha$-Pinene to generate $\mathrm{THz}$ waves, it is essential to ensure its weak absorption property within the frequency region of interest.

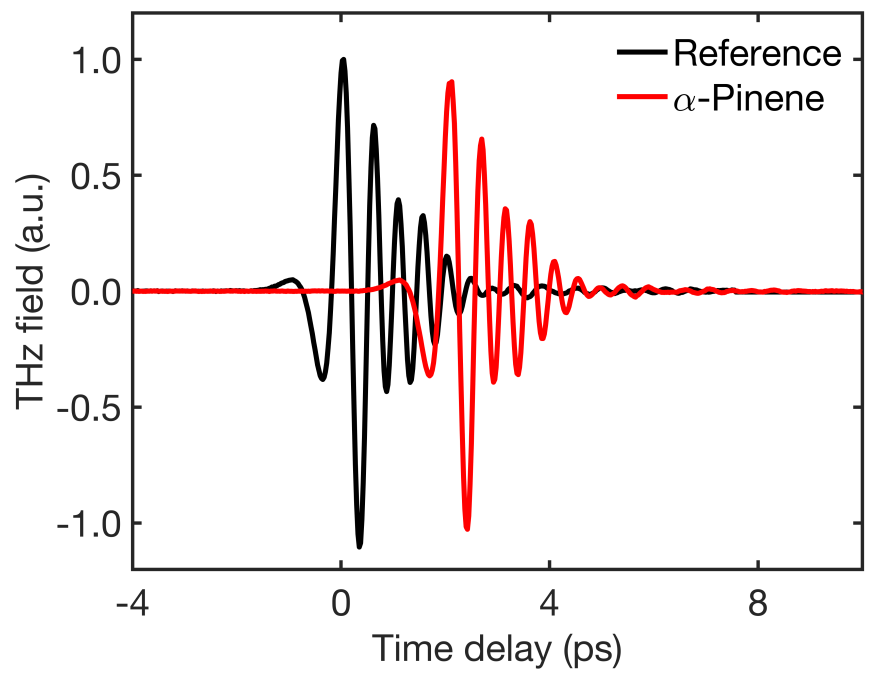

Figure 5.1: Waveform measurement of $\alpha$-Pinene as the sample by using a standard THz-TDS system. The black curve is the reference waveform with no sample presented.

A standard THz-TDS system was used for measurement [104]. The red curve in Figure 5.11 shows the $\mathrm{THz}$ waveform with a $1 \mathrm{~mm}$ thick $\alpha$-Pinene sample. As a reference, the black curve is the measured $\mathrm{THz}$ waveform without the sample. Similar to the discussion in Section 1.2 , the different field amplitude results from the absorption of $\mathrm{THz}$ waves, the time shift is caused by the different refractive indices of the air and the sample. The information in the frequency domain can be obtained through the Fourier

\footnotetext{
${ }^{1}$ Data was provided by Dr. Yiwen E.
} 
transform, which is utilized to calculate the frequency-dependent index of refraction and field absorption coefficient of the sample. The result is shown in Figure 5.2 .

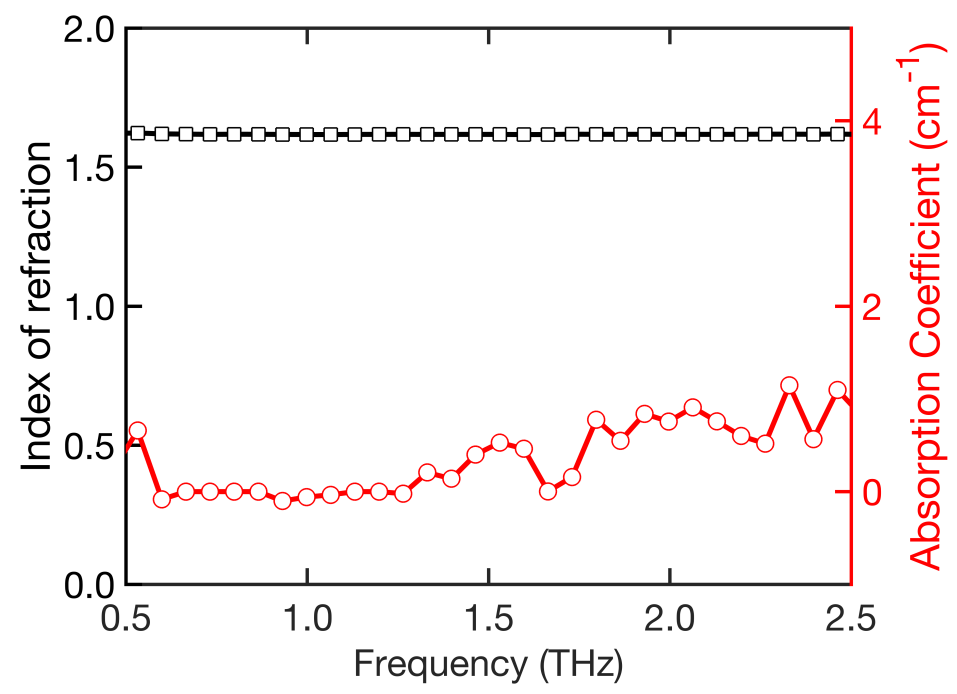

Figure 5.2: Measured results of refractive index and field absorption coefficient of $\alpha$-Pinene in the frequency region $0.5-2.5 \mathrm{THz}$.

The black squares in Figure 5.2 plot the index of refraction versus the frequency. $\alpha$-Pinene has a refractive index of 1.62 from $0.5 \mathrm{THz}$ to $2.5 \mathrm{THz}$ with little dispersion. The red circles show that the field absorption coefficient of $\alpha$-Pinene in such a frequency regime is less than $2 \mathrm{~cm}^{-1}$. A few points showing negative values of absorption coefficient may result from system error when the absorption is very weak. Compared with the value of water (see Figure 1.9), the absorption coefficient of $\alpha$-Pinene is impressively small. 


\subsection{Comparison between Terahertz Radiation from $\alpha$-Pinene and Water}

Since the absorption of $\alpha$-Pinene is much weaker than that of water, a stronger THz signal is expected to be obtained from $\alpha$-Pinene. It is worth underlining that $\alpha$-Pinene is too viscous to be used to form a stable freeflowing film for the generation of $\mathrm{THz}$ waves. Thus, a syringe needle was applied to form a forced-flowing $\alpha$-Pinene line. The experimental setup is the same as the one described in Section 4.2 . The diameter of the liquid line is $210 \mu \mathrm{m}$. Laser pulse energy is $0.4 \mathrm{~mJ}$ with the optimal pulse duration around $345 \mathrm{fs}$. THz radiation generated from an $\alpha$-Pinene line and a water line were recorded for comparison. Figure 5.3 (a) shows the measured time domain waveforms. Their Fourier transform offers the information in the frequency domain (see Figure 5.3(b)).

Figure 5.3 shows that $\alpha$-Pinene emits a stronger THz field, which is about 1.8-times stronger than that from water in the identical experimental condition (liquid lines diameter, laser pulse energy, laser pulse duration, etc.). The electric field strength of $\mathrm{THz}$ waves generated by the $210 \mu \mathrm{m}$ diameter $\alpha$ Pinene line is estimated to be $0.7 \mathrm{kV} / \mathrm{cm}$ through EOS [52]. In the frequency domain, $\alpha$-Pinene offers more high-frequency (above $1 \mathrm{THz}$ ) components and provides a wider bandwidth.

The significant difference in absorption characteristics between water and $\alpha$-Pinene is the main reason that causes their difference in Figure 5.3. To see 
(a)
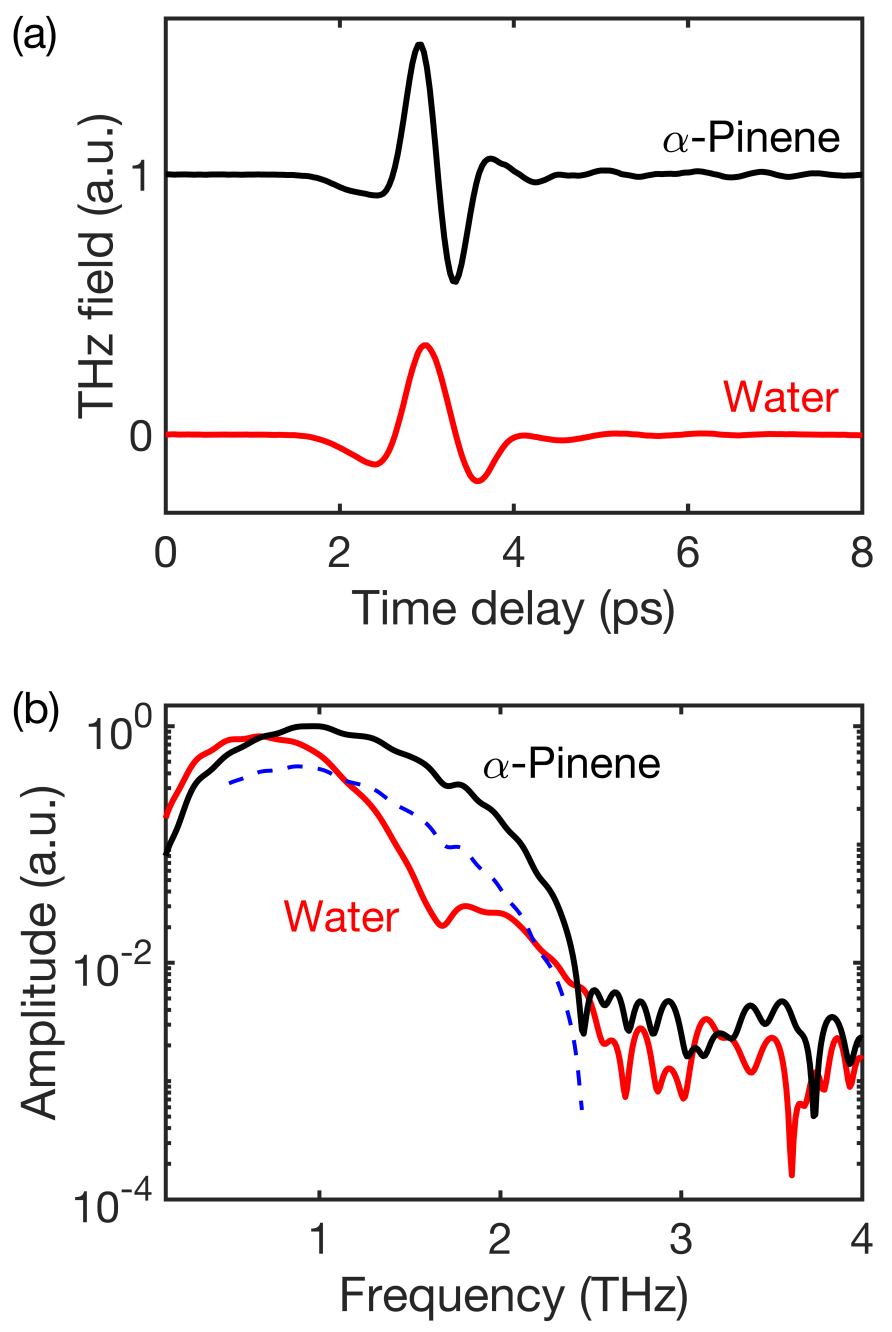

Figure 5.3: Comparison of $\mathrm{THz}$ waves generated from $\alpha$-Pinene and water in (a) time domain and (b) frequency domain. The diameter of the liquid line is $210 \mu \mathrm{m}$. Laser pulse energy is $0.4 \mathrm{~mJ}$. Optical pulse durations are individually optimized for $\alpha$-Pinene and water. Both have a value around $345 \mathrm{fs}$. The dash line in (b) is calculated by removing the absorption of $\alpha$ Pinene and adding the absorption of water to the black curve from $0.5 \mathrm{THz}$ to $2.5 \mathrm{THz}$. 
whether it is the only contributor or not, the absorption from $\alpha$-Pinene is removed from the experimental data (black curve) and the absorption from water is added [4]. This treatment of ignoring the change of absorption caused by the plasma formation is justified because only $0.2 \%$ of molecules are ionized in this case. The influence of their different refractive indices on the Fresnel coefficients of the interfaces is included. The result is plotted as the blue dash line in Figure 5.3(b). The discrepancy between the blue dash line and the red curve reveals that there are other contributors involved.

Learnt from the discussion in Section 2.8, many other parameters (such as refractive index, ionization energy, ionization time, collision time, molecule mass, and molecular density) of liquids function in the ionization process, and thus may affect the $\mathrm{THz}$ radiation resulting in the observation in Figure 5.3(b). Further investigation into plasma current [105, 106] and plasma radiation [107] for THz waves is needed to fully understand the result in Figure 5.3 .

\subsection{Summary}

THz wave generation from $\alpha$-Pinene is presented. From the experimental results, $\alpha$-Pinene generates a stronger $\mathrm{THz}$ signal with a wider bandwidth than water does, which is mainly due to its weak absorption characteristic for THz waves. From the author's point of view, other material-related parameters, such as refractive index, ionization energy, ionization time, collision 
time, molecule mass, and molecular density of liquids can also influence on the generation of $\mathrm{THz}$ waves.

In addition, many other liquids have also been tested for the generation of $\mathrm{THz}$ waves. Heavy water, salty water, ethanol (different concentration), milk (different fat), and p-xylene can also be used to emit THz waves. Further detailed discussions about $\mathrm{THz}$ wave generation from different liquids are beyond the scope of this dissertation. 


\section{Chapter 6}

\section{Conclusion and Future Work}

This dissertation focuses on the investigation into using liquid water to gen-

erate $\mathrm{THz}$ radiation, which is called as "Terahertz Aqueous Photonics". It should be noted that laser-induced plasma in water plays a significant role in the generation process even though the source is originally in the liquid form. This work not only paves the way for developing intense $\mathrm{THz}$ sources but also has contributed to enthralling insight into the study of laser-water interaction.

Broadband $\mathrm{THz}$ wave generation from a water film was first demonstrated. Mechanism of the generation process is attributed to laser-induced plasma formation in water associated with a ponderomotive force-induced dipole radiation model. Specifically, it is observed that $\mathrm{THz}$ radiation from liquid water prefers sub-picosecond laser pulses for the excitation, which can be fully explained by the process of laser-induced plasma formation in water. Furthermore, measurement of the $\mathrm{THz}$ radiation pattern matches well with the simulation predicted by the dipole radiation model. 
THz emission from a water film under the two-color excitation scheme was also investigated. One-order of magnitude stronger $\mathrm{THz}$ electric field is obtained by using the asymmetric excitation scheme with short temporal laser pulses. The modulation of $\mathrm{THz}$ waves is achieved by adjusting the relative phase between $\omega$ and $2 \omega$ pulses. The method also enables the observation of modulated and unmodulated $\mathrm{THz}$ waves with a Golay cell. By increasing the excitation pulse energy, the unmodulated component is linearly raised while the modulated component is quadratically enhanced above the threshold, which indicates that the $\mathrm{THz}$ yield can be dramatically scaled up by increasing the excitation pulse energy.

To mitigate the total internal reflection at the water-air interface of water films, water lines produced by syringe needles were applied to achieve stronger $\mathrm{THz}$ signals. It is found that the optimal laser pulse duration highly depends upon the diameter of the water line, which also impacts on the $\mathrm{THz}$ radiation energy. The maximum $\mathrm{THz}$ signal is obtained with a $210 \mu \mathrm{m}$ diameter water line.

As a supplement to the study of Terahertz Aqueous Photonics, THz wave generation from $\alpha$-Pinene was presented. $\alpha$-Pinene generates a 1.8-times stronger $\mathrm{THz}$ electric field with a wider bandwidth than water does. The stronger $\mathrm{THz}$ radiation mainly results from the fact that $\alpha$-Pinene almost does not absorb $\mathrm{THz}$ waves.

It needs to be highlighted that $\mathrm{THz}$ wave generation from the water is stronger than that from the air with the one-color excitation scheme, which 
is coincident as the expectation from the higher molecule density of water. However, water is no longer the winner with the two-color excitation scheme. In water, cascade ionization favors a long pulse duration (sub-picosecond) to exponentially produce electrons. But the second-harmonic generation efficiency from the fundamental laser pulse significantly drops as the optical pulse duration increases. The discrepant demand regarding the pulse duration significantly limits the generation of $\mathrm{THz}$ waves under the two-color excitation. However, from the perspective of the development of intense liquid $\mathrm{THz}$ sources, the two-color excitation scheme may still be the way to go. Wise management of laser pulse duration is imperative. It should be noticed that only a few types of liquids have been tested so far. The best liquid source for THz radiation may still be out of the scope. Systematical tests of other liquids are necessary. Commercializing a compact liquid $\mathrm{THz}$ source is an interesting and promising topic as well. Moreover, developing a liquid $\mathrm{THz}$ sensor can be a challenging project to further the research of Terahertz Aqueous Photonics. 


\section{Bibliography}

[1] Kaori Fukunaga and Marcello Picollo. Terahertz spectroscopy applied to the analysis of artists' materials. Applied Physics A, 100(3):591-597, 2010 .

[2] VP Kandidov, OG Kosareva, IS Golubtsov, W Liu, A Becker, N Akozbek, CM Bowden, and SL Chin. Self-transformation of a powerful femtosecond laser pulse into a white-light laser pulse in bulk optical media (or supercontinuum generation). Applied Physics B, 77(2-3):149165, 2003.

[3] A Flettner, T Pfeifer, D Walter, C Winterfeldt, C Spielmann, and G Gerber. High-harmonic generation and plasma radiation from water microdroplets. Applied Physics B, 77(8):747-751, 2003.

[4] Cecilie Ro/nne, Lars Thrane, Per-Olof Åstrand, Anders Wallqvist, Kurt V Mikkelsen, and So/ren R Keiding. Investigation of the temperature dependence of dielectric relaxation in liquid water by thz reflection spectroscopy and molecular dynamics simulation. The Journal of Chemical Physics, 107(14):5319-5331, 1997.

[5] Tianwu Wang, Pernille Klarskov, and Peter Uhd Jepsen. Ultrabroadband $\mathrm{THz}$ time-domain spectroscopy of a free-flowing water film. IEEE Transactions on Terahertz Science and Technology, 4(4):425-431, 2014.

[6] Xu Xie, Jianming Dai, and X. -C. Zhang. Coherent control of THz wave generation in ambient air. Physical Review Letters, 96(7):075005, 2006 . 
[7] Jianming Dai, Nicholas Karpowicz, and X. -C. Zhang. Coherent polarization control of terahertz waves generated from two-color laserinduced gas plasma. Physical Review Letters, 103(2):023001, 2009.

[8] X. -C. Zhang and Jingzhou Xu. Introduction to THz Wave Photonics, volume 29. Springer, 2010.

[9] Kai-Erik Peiponen, Axel Zeitler, and Makoto Kuwata-Gonokami. Terahertz Spectroscopy and Imaging, volume 171. Springer, 2012.

[10] Yun-Shik Lee. Principles of Terahertz Science and Technology, volume 170. Springer Science \& Business Media, 2009.

[11] Daniel M. Mittleman. Twenty years of terahertz imaging. Optics Express, 26(8):9417-9431, 2018.

[12] Jiapeng Zhao, E Yiwen, Kaia Williams, X. -C. Zhang, and Robert W Boyd. Spatial sampling of terahertz fields with sub-wavelength accuracy via probe-beam encoding. Light: Science \& Applications, 8(1):55, 2019.

[13] David C Look. Molecular beam epitaxial gaas grown at low temperatures. Thin Solid Films, 231(1-2):61-73, 1993.

[14] Matthew C Beard, Gordon M Turner, and Charles A Schmuttenmaer. Subpicosecond carrier dynamics in low-temperature grown gaas as measured by time-resolved terahertz spectroscopy. Journal of Applied Physics, 90(12):5915-5923, 2001.

[15] DH Auston, KP Cheung, and PR Smith. Picosecond photoconducting hertzian dipoles. Applied Physics Letters, 45(3):284-286, 1984.

[16] Robert W Boyd. Nonlinear Optics. Elsevier, 2003.

[17] Janos Hebling, Gabor Almasi, Ida Z Kozma, and Jürgen Kuhl. Velocity matching by pulse front tilting for large-area THz-pulse generation. Optics Express, 10(21):1161-1166, 2002.

[18] János Hebling, Ka-Lo Yeh, Matthias C Hoffmann, Balázs Bartal, and Keith A Nelson. Generation of high-power terahertz pulses by tiltedpulse-front excitation and their application possibilities. JOSA B, 25(7):B6-B19, 2008. 
[19] JA Fülöp, L Pálfalvi, S Klingebiel, G Almási, F Krausz, S Karsch, and $\mathrm{J}$ Hebling. Generation of sub-mJ terahertz pulses by optical rectification. Optics Letters, 37(4):557-559, 2012.

[20] X. -C. Zhang, XF Ma, Y Jin, T-M Lu, EP Boden, PD Phelps, KR Stewart, and CP Yakymyshyn. Terahertz optical rectification from a nonlinear organic crystal. Applied Physics Letters, 61(26):3080-3082, 1992.

[21] Christoph P Hauri, Clemens Ruchert, Carlo Vicario, and Fernando Ardana. Strong-field single-cycle $\mathrm{THz}$ pulses generated in an organic crystal. Applied Physics Letters, 99(16):161116, 2011.

[22] Mostafa Shalaby and Christoph P Hauri. Demonstration of a lowfrequency three-dimensional terahertz bullet with extreme brightness. Nature Communications, 6:5976, 2015.

[23] H. Hamster, A. Sullivan, S. Gordon, W. White, and R. W. Falcone. Subpicosecond, electromagnetic pulses from intense laser-plasma interaction. Physical Review Letters, 71:2725-2728, 1993.

[24] H. Hamster, A. Sullivan, S. Gordon, and R. W. Falcone. Short-pulse terahertz radiation from high-intensity-laser-produced plasmas. Physical Review E, 49:671-677, 1994.

[25] D. J. Cook and R. M. Hochstrasser. Intense terahertz pulses by fourwave rectification in air. Optics Letters, 25(16):1210-1212, 2000.

[26] Keith Johnson, Matthew Price-Gallagher, Orval Mamer, Alain Lesimple, Clark Fletcher, Yunqing Chen, Xiaofei Lu, Masashi Yamaguchi, and X. -C. Zhang. Water vapor: an extraordinary terahertz wave source under optical excitation. Physics Letters A, 372(38):6037-6040, 2008.

[27] Ki-Yong Kim, James H Glownia, Antoinette J Taylor, and George Rodriguez. Terahertz emission from ultrafast ionizing air in symmetrybroken laser fields. Optics Express, 15(8):4577-4584, 2007.

[28] Ki-Yong Kim, AJ Taylor, JH Glownia, and G Rodriguez. Coherent control of terahertz supercontinuum generation in ultrafast laser-gas interactions. Nature Photonics, 2(10):605, 2008. 
[29] Ki-Yong Kim. Generation of coherent terahertz radiation in ultrafast laser-gas interactions. Physics of Plasmas, 16(5):056706, 2009.

[30] Nicholas Karpowicz and X. -C. Zhang. Coherent terahertz echo of tunnel ionization in gases. Physical Review Letters, 102(9):093001, 2009.

[31] Tobias Kampfrath, Marco Battiato, Pablo Maldonado, G Eilers, J Nötzold, Sebastian Mährlein, V Zbarsky, F Freimuth, Y Mokrousov, S Blügel, et al. Terahertz spin current pulses controlled by magnetic heterostructures. Nature Nanotechnology, 8(4):256, 2013.

[32] Tom Seifert, S Jaiswal, U Martens, J Hannegan, Lukas Braun, Pablo Maldonado, F Freimuth, A Kronenberg, J Henrizi, I Radu, et al. Efficient metallic spintronic emitters of ultrabroadband terahertz radiation. Nature Photonics, 10(7):483, 2016.

[33] L Thrane, Rune Hylsberg Jacobsen, P Uhd Jepsen, and SR Keiding. $\mathrm{THz}$ reflection spectroscopy of liquid water. Chemical Physics Letters, 240(4):330-333, 1995.

[34] John C Kotz, Paul M Treichel, and John Townsend. Chemistry and Chemical Reactivity. Cengage Learning, 2012.

[35] D Engels, J Schmid-Burgk, and CM Walmsley. Water maser emission from OH/IR stars. Astronomy and Astrophysics, 167:129-144, 1986.

[36] David A Neufeld and Gary J Melnick. Excitation of millimeter and submillimeter water masers. The Astrophysical Journal, 368:215-230, 1991.

[37] David A Neufeld, Philip R Maloney, and Sarah Conger. Water maser emission from X-ray-heated circumnuclear gas in active galaxies. The Astrophysical Journal, 436:L-127, 1994.

[38] Robert R Alfano and SL Shapiro. Observation of self-phase modulation and small-scale filaments in crystals and glasses. Physical Review Letters, 24(11):592, 1970.

[39] Takahashi Jimbo, VL Caplan, Qing-Xin Li, QZ Wang, Ping Pei Ho, and RR Alfano. Enhancement of ultrafast supercontinuum generation in water by the addition of $\mathrm{Zn} 2+$ and $\mathrm{K}+$ cations. Optics Letters, 12(7):477-479, 1987. 
[40] W Liu, S Petit, A Becker, N Aközbek, CM Bowden, and SL Chin. Intensity clamping of a femtosecond laser pulse in condensed matter. Optics Communications, 202(1-3):189-197, 2002.

[41] AK Dharmadhikari, FA Rajgara, and D Mathur. Systematic study of highly efficient white light generation in transparent materials using intense femtosecond laser pulses. Applied Physics B, 80(1):61-66, 2005.

[42] N Kaya, J Strohaber, AA Kolomenskii, G Kaya, Hartmut Schröder, and HA Schuessler. White-light generation using spatially-structured beams of femtosecond radiation. Optics Express, 20(12):13337-13346, 2012 .

[43] JA Dharmadhikari, G Steinmeyer, G Gopakumar, D Mathur, and AK Dharmadhikari. Femtosecond supercontinuum generation in water in the vicinity of absorption bands. Optics Letters, 41(15):3475-3478, 2016.

[44] David Attwood and Anne Sakdinawat. X-rays and Extreme Ultraviolet Radiation: Principles and Applications. Cambridge University Press, 2017.

[45] SJ McNaught, J Fan, E Parra, and HM Milchberg. A pump-probe investigation of laser-droplet plasma dynamics. Applied Physics Letters, 79(25):4100-4102, 2001.

[46] St Düsterer, H Schwoerer, W Ziegler, C Ziener, and R Sauerbrey. Optimization of EUV radiation yield from laser-produced plasma. Applied Physics B, 73(7):693-698, 2001.

[47] Heiko G Kurz, Daniel S Steingrube, Detlev Ristau, Manfred Lein, Uwe Morgner, and Milutin Kovačev. High-order-harmonic generation from dense water microdroplets. Physical Review A, 87(6):063811, 2013.

[48] David N Nikogosyan, Alexander A Oraevsky, and Valery I Rupasov. Two-photon ionization and dissociation of liquid water by powerful laser UV radiation. Chemical Physics, 77(1):131-143, 1983.

[49] Robert A Crowell and David M Bartels. Multiphoton ionization of liquid water with 3.0-5.0 eV photons. The Journal of Physical Chemistry, 100(45):17940-17949, 1996. 
[50] Paul K Kennedy. A first-order model for computation of laser-induced breakdown thresholds in ocular and aqueous media. I. Theory. IEEE Journal of Quantum Electronics, 31(12):2241-2249, 1995.

[51] Paul K Kennedy, Daniel X Hammer, and Benjamin A Rockwell. Laserinduced breakdown in aqueous media. Progress in Quantum Electronics, 21(3):155-248, 1997.

[52] H Hirori, A Doi, F Blanchard, and K Tanaka. Single-cycle terahertz pulses with amplitudes exceeding $1 \mathrm{MV} / \mathrm{cm}$ generated by optical rectification in $\mathrm{LiNbO}_{3}$. Applied Physics Letters, 98(9):091106, 2011.

[53] F Blanchard, L Razzari, H-C Bandulet, G Sharma, R Morandotti, J-C Kieffer, T Ozaki, M Reid, HF Tiedje, HK Haugen, et al. Generation of $1.5 \mu \mathrm{J}$ single-cycle terahertz pulses by optical rectification from a large aperture ZnTe crystal. Optics Express, 15(20):13212-13220, 2007.

[54] Q. Wu and X. -C. Zhang. Free-space electro-optic sampling of terahertz beams. Applied Physics Letters, 67(24):3523-3525, 1995.

[55] Liang-Liang Zhang, Wei-Min Wang, Tong Wu, Shi-Jia Feng, Kai Kang, Cun-Lin Zhang, Yan Zhang, Yu-Tong Li, Zheng-Ming Sheng, and X. C Zhang. Strong terahertz radiation from a liquid-water line. Physical Review Applied, 12(1):014005, 2019.

[56] W-M Wang, Paul Gibbon, Z-M Sheng, and Y-T Li. Integrated simulation approach for laser-driven fast ignition. Physical Review E, 91(1):013101, 2015.

[57] Fabrizio Buccheri and X. -C. Zhang. Terahertz emission from laserinduced microplasma in ambient air. Optica, 2(4):366-369, 2015.

[58] Jian-Zhi Zhang, Joseph K. Lam, Carol F. Wood, Boa-Teh Chu, and Richard K. Chang. Explosive vaporization of a large transparent droplet irradiated by a high intensity laser. Applied Optics, 26(22):4731-4737, 1987.

[59] Chris B. Schaffer, Nozomi Nishimura, Eli N. Glezer, Albert M.-T. Kim, and Eric Mazur. Dynamics of femtosecond laser-induced breakdown in water from femtoseconds to microseconds. Optics Express, 10(3):196203, 2002. 
[60] François Courvoisier, Véronique Boutou, Catherine Favre, Steven C. Hill, and Jean-Pierre Wolf. Plasma formation dynamics within a water microdroplet on femtosecond time scales. Optics Letters, 28(3):206208, Feb 2003.

[61] Albrecht Lindinger, Jan Hagen, Liana D. Socaciu, Thorsten M. Bernhardt, Ludger Wöste, Denis Duft, and Thomas Leisner. Time-resolved explosion dynamics of $\mathrm{H}_{2} \mathrm{O}$ droplets induced by femtosecond laser pulses. Applied Optics, 43(27):5263-5269, 2004.

[62] Claudiu A. Stan, Despina Milathianaki, Hartawan Laksmono, Raymond G. Sierra, Trevor A. McQueen, Marc Messerschmidt, Garth J. Williams, Jason E. Koglin, Thomas J. Lane, Matt J. Hayes, Serge A.H. Guillet, Mengning Liang, Andrew L. Aquila, Philip R. Willmott, Joseph S. Robinson, Karl L. Gumerlock, Sabine Botha, Karol Nass, Ilme Schlichting, Robert L. Shoeman, Howard A. Stone, and Sébastien Boutet. Liquid explosions induced by X-ray laser pulses. Nature Physics, 12(10):966-971, 102016.

[63] H Barry Bebb and Albert Gold. Multiphoton ionization of hydrogen and rare-gas atoms. Physical Review, 143(1):1, 1966.

[64] Claudio DeMichelis. Laser induced gas breakdown: A bibliographical review. IEEE Journal of Quantum Electronics, 5(4):188-202, 1969.

[65] Yuen-Ron Shen. The principles of nonlinear optics. New York, WileyInterscience, 1984,575 p., 1984.

[66] P Lambropoulos. Mechanisms for multiple ionization of atoms by strong pulsed lasers. Physical Review Letters, 55(20):2141, 1985.

[67] MD Perry, OL Landen, A Szöke, and EM Campbell. Multiphoton ionization of the noble gases by an intense $10^{14}-\mathrm{W} / \mathrm{cm}^{2}$ dye laser. Physical Review A, 37(3):747, 1988.

[68] LV Keldysh et al. Ionization in the field of a strong electromagnetic wave. Soviet Physics JETP, 20(5):1307-1314, 1965.

[69] MV Ammosov, NB Delone, V Krainov, Am Perelomov, V Popov, M Terent'ev, Gennady L Yudin, and Misha Yu Ivanov. Tunnel ionization of complex atoms and of atomic ions in an alternating electric field. Sov. Phys. JETP, 64(1191):26, 1986. 
[70] Michael Bass and H Barrett. Avalanche breakdown and the probabilistic nature of laser-induced damage. IEEE Journal of Quantum Electronics, 8(3):338-343, 1972.

[71] Nicolaas Bloembergen. Laser-induced electric breakdown in solids. IEEE Journal of Quantum Electronics, 10(3):375-386, 1974.

[72] C Grey Morgan. Laser-induced breakdown of gases. Reports on Progress in Physics, 38(5):621, 1975.

[73] C Puliafito and R Steinert. Short-pulsed Nd: YAG laser microsurgery of the eye: biophysical considerations. IEEE Journal of Quantum Electronics, 20(12):1442-1448, 1984.

[74] Joachim Noack and Alfred Vogel. Laser-induced plasma formation in water at nanosecond to femtosecond time scales: calculation of thresholds, absorption coefficients, and energy density. IEEE Journal of Quantum Electronics, 35(8):1156-1167, 1999.

[75] Ferd Williams, SP Varma, and S Hillenius. Liquid water as a lonepair amorphous semiconductor. The Journal of Chemical Physics, 64(4):1549-1554, 1976.

[76] CA Sacchi. Laser-induced electric breakdown in water. JOSA B, 8(2):337-345, 1991.

[77] Q Feng, Jerome V Moloney, Alan C Newell, Ewan M Wright, Kennedy Cook, Paul K Kennedy, DX Hammer, BA Rockwell, and CR Thompson. Theory and simulation on the threshold of water breakdown induced by focused ultrashort laser pulses. IEEE Journal of Quantum Electronics, 33(2):127-137, 1997.

[78] MD Feit and JA Fleck Jr. Effect of refraction on spot-size dependence of laser-induced breakdown. Applied Physics Letters, 24(4):169-172, 1974 .

[79] Arnaud Couairon and André Mysyrowicz. Femtosecond filamentation in transparent media. Physics Reports, 441(2-4):47-189, 2007.

[80] Yurii P Raizer. Reviews of topical problems: Breakdown and heating of gases under the influence of a laser beam. Soviet Physics Uspekhi, 8:650-673, 1966. 
[81] Jianming Dai, Jingle Liu, and X. -C Zhang. Terahertz wave air photonics: terahertz wave generation and detection with laser-induced gas plasma. IEEE Journal of Selected Topics in Quantum Electronics, 17(1):183-190, 2010.

[82] Markus Kreß, Torsten Löffler, Mark D Thomson, Reinhard Dörner, Hartmut Gimpel, Karl Zrost, Thorsten Ergler, Robert Moshammer, Uwe Morgner, Joachim Ullich, et al. Determination of the carrierenvelope phase of few-cycle laser pulses with terahertz-emission spectroscopy. Nature Physics, 2(5):327, 2006.

[83] P Gaal, W Kuehn, K Reimann, M Woerner, T Elsaesser, and R Hey. Internal motions of a quasiparticle governing its ultrafast nonlinear response. Nature, 450(7173):1210, 2007.

[84] HG Roskos, MD Thomson, M Kreß, Löffler, and T. Broadband THz emission from gas plasmas induced by femtosecond optical pulses: From fundamentals to applications. Laser \& Photonics Reviews, 1(4):349368, 2007.

[85] TI Oh, YJ Yoo, YS You, and KY Kim. Generation of strong terahertz fields exceeding $8 \mathrm{MV} / \mathrm{cm}$ at $1 \mathrm{kHz}$ and real-time beam profiling. Applied Physics Letters, 105(4):041103, 2014.

[86] Mark D Thomson, Volker Blank, and Hartmut G Roskos. Terahertz white-light pulses from an air plasma photo-induced by incommensurate two-color optical fields. Optics Express, 18(22):23173-23182, 2010.

[87] X. -C Zhang, Alexander Shkurinov, and Yan Zhang. Extreme terahertz science. Nature Photonics, 11(1):16, 2017.

[88] Haidan Wen and Aaron M Lindenberg. Coherent terahertz polarization control through manipulation of electron trajectories. Physical Review Letters, 103(2):023902, 2009.

[89] Jianming Dai and X. -C Zhang. Terahertz wave generation from thin metal films excited by asymmetrical optical fields. Optics Letters, 39(4):777-780, 2014.

[90] Indranuj Dey, Kamalesh Jana, Vladimir Yu Fedorov, Anastasios D Koulouklidis, Angana Mondal, Moniruzzaman Shaikh, Deep Sarkar, 
Amit D Lad, Stelios Tzortzakis, Arnaud Couairon, et al. Highly efficient broadband terahertz generation from ultrashort laser filamentation in liquids. Nature Communications, 8(1):1184, 2017.

[91] Y Shen, T Watanabe, DA Arena, C-C Kao, JB Murphy, TY Tsang, XJ Wang, and GL Carr. Nonlinear cross-phase modulation with intense single-cycle terahertz pulses. Physical Review Letters, 99(4):043901, 2007.

[92] Dmitry Turchinovich, Jørn M Hvam, and Matthias C Hoffmann. Selfphase modulation of a single-cycle terahertz pulse by nonlinear freecarrier response in a semiconductor. Physical Review B, 85(20):201304, 2012 .

[93] Emilio A Nanni, Wenqian R Huang, Kyung-Han Hong, Koustuban Ravi, Arya Fallahi, Gustavo Moriena, RJ Dwayne Miller, and Franz X Kärtner. Terahertz-driven linear electron acceleration. Nature Communications, 6:8486, 2015.

[94] Dongfang Zhang, Arya Fallahi, Michael Hemmer, Xiaojun Wu, Moein Fakhari, Yi Hua, Huseyin Cankaya, Anne-Laure Calendron, Luis E Zapata, Nicholas H Matlis, et al. Segmented terahertz electron accelerator and manipulator (STEAM). Nature Photonics, 12(6):336, 2018.

[95] Qi Jin, Yiwen E, Kaia Williams, Jianming Dai, and X. -C Zhang. Observation of broadband terahertz wave generation from liquid water. Applied Physics Letters, 111(7):071103, 2017.

[96] P Prem Kiran, Suman Bagchi, Siva Rama Krishnan, CL Arnold, G Ravindra Kumar, and Arnaud Couairon. Focal dynamics of multiple filaments: Microscopic imaging and reconstruction. Physical Review A, 82(1):013805, 2010.

[97] Xiao-Long Liu, Xin Lu, Xun Liu, Ting-Ting Xi, Feng Liu, Jing-Long Ma, and Jie Zhang. Tightly focused femtosecond laser pulse in air: from filamentation to breakdown. Optics Express, 18(25):26007-26017, 2010.

[98] See Leang Chin. Femtosecond Laser Filamentation, volume 55. Springer, 2010. 
[99] F Docchio. Lifetimes of plasmas induced in liquids and ocular media by single Nd: YAG laser pulses of different duration. EPL (Europhysics Letters), 6(5):407, 1988.

[100] Q Feng, Jerome V Moloney, Alan C Newell, and Ewan M Wright. Laser-induced breakdown versus self-focusing for focused picosecond pulses in water. Optics Letters, 20(19):1958-1960, 1995.

[101] Jianming Dai and X. -C Zhang. Terahertz wave generation from gas plasma using a phase compensator with attosecond phase-control accuracy. Applied Physics Letters, 94(2):021117, 2009.

[102] E Yiwen, Qi Jin, Anton Tcypkin, and X. -C Zhang. Terahertz wave generation from liquid water films via laser-induced breakdown. Applied Physics Letters, 113:181103, 2018.

[103] Qi Jin, Jianming Dai, Yiwen E, and X. -C Zhang. Terahertz wave emission from a liquid water film under the excitation of asymmetric optical fields. Applied Physics Letters, 113(26):261101, 2018.

[104] Timothy D Dorney, Richard G Baraniuk, and Daniel M Mittleman. Material parameter estimation with terahertz time-domain spectroscopy. JOSA A, 18(7):1562-1571, 2001.

[105] Ihar Babushkin, Wilhelm Kuehn, Christian Koehler, Stefan Skupin, Luc Berge, Klaus Reimann, Michael Woerner, Joachim Herrmann, and Thomas Elsaesser. Ultrafast spatiotemporal dynamics of terahertz generation by ionizing two-color femtosecond pulses in gases. Physical Review Letters, 105(5):053903, 2010.

[106] Luc Bergé, Stefan Skupin, Christian Köhler, Ihar Babushkin, and Joachim Herrmann. 3d numerical simulations of thz generation by twocolor laser filaments. Physical Review Letters, 110(7):073901, 2013.

[107] Phillip Sprangle, JR Penano, Bahman Hafizi, and CA Kapetanakos. Ultrashort laser pulses and electromagnetic pulse generation in air and on dielectric surfaces. Physical Review E, 69(6):066415, 2004. 